\title{
AN EXPERIMENTAL AND EXPERIENCE SAMPLING STUDY OF EMOTION IN PEOPLE WITH GENERALIZED ANXIETY DISORDER
}

by

\author{
Elizabeth J. Pawluk
}

Master of Arts, Ryerson University, 2011

Bachelor of Arts, University of Western Ontario, 2008

\author{
A dissertation \\ presented to Ryerson University \\ in partial fulfillment of the \\ requirements for the degree of \\ Doctor of Philosophy \\ in the Program of \\ Psychology
}

Toronto, Ontario, Canada, 2016

C Elizabeth J. Pawluk 2016 


\section{Author's Declaration for Electronic Submission of Dissertation}

I hereby declare that I am the sole author of this dissertation. This is a true copy of the dissertation, including any required final revisions, as accepted by my examiners.

I authorize Ryerson University to lend this dissertation to other institutions or individuals for the purpose of scholarly research.

I further authorize Ryerson University to reproduce this dissertation by photocopying or by other means, in total or in part, at the request of other institutions or individuals for the purpose of scholarly research.

I understand that my dissertation may be made electronically available to the public. 


\begin{abstract}
An Experimental and Experience Sampling Study of Emotion in People with Generalized Anxiety Disorder
\end{abstract}

Doctor of Philosophy, 2016

Elizabeth J. Pawluk

Psychology

Ryerson University

Difficulties with emotion and emotion regulation have a significant role in generalized anxiety disorder (GAD), however, much remains unknown about the emotional profile of people with GAD. The emotion dysregulation model (Mennin et al., 2005) suggests that people with GAD experience emotions at a heightened intensity and use maladaptive strategies, including worry, to regulate their distress. This dissertation comprises two independent studies to test tenets of the model. Study 1 was a laboratory-based experiment to clarify if the heightened emotional intensity experienced by people with GAD is due to baseline arousal or emotional reactivity. The subjective emotional and physiological responses of people with GAD $(n=22)$ were compared to those of people with social anxiety disorder (SAD; $n=23)$ and nonclinical controls (NCC; $n=$ 20) at baseline and following an emotion induction. The GAD group reported greater subjective intensity of negative emotions and lower intensity of positive emotions relative to the control groups. No differences were found across the three groups in their level of emotional reactivity. The findings highlight that baseline intensity, not emotional reactivity, accounts for the heightened emotional intensity reported by people with GAD. Study 2 was an experience sampling study to identify the emotional profile of people with GAD and examine direct effect of worry and of external events on negative and positive emotions in daily life. Relative to the 
NCC group ( $n=41)$, the GAD group $(n=39)$ exhibited an emotional profile characterized by elevated mean intensity, greater instability and greater inertia of negative emotions and lower mean intensity, greater instability and lower inertia of positive emotions. Worry had a greater negative effect on the emotions reported by the GAD group relative to those of the NCC group. Finally, the groups did not differ in degree of emotional reactivity to negative events, but the GAD group reported a greater increase in positive emotion and a greater decrease in negative emotion following a positive event compared to the NCC group. Overall, the findings inform the emotion dysregulation model and provide unique insights into the dynamic emotional experiences of those with GAD. 


\section{Acknowledgements}

I would like to express my sincere gratitude to Dr. Naomi Koerner, my supervisor. Thank you for sharing your passion for and steadfast commitment to innovative and high-quality research with me over the past 7 years. Your encouragement and support to pursue my ideas allowed me to extend and push through my knowledge boundaries. I would also like to thank Drs. Martin Antony and Janice Kuo, members of my internal supervisory committee. Thank you for your guidance, insights, and support throughout the phases of the project. I also wish to thank the external members of my examining committee, Drs. Chris MacDonald and Henny Westra, for their interest in my dissertation and willingness to participate in my defense.

I am grateful to my parents for their unconditional love and for all that they have provided me. Your words of wisdom and support have encouraged me to pursue my passions and kept me motivated even through the most challenging times. To James, my soon-to-be husband, thank you for being my rock. I am excited to start the next chapter of life with you. To my labmates and friends, thank you for your ever-available support. Finally, I would like to acknowledge my external funding sources the Canadian Institutes of Health Research and the Ontario Graduate Scholarship Program. 


\section{Table of Contents}

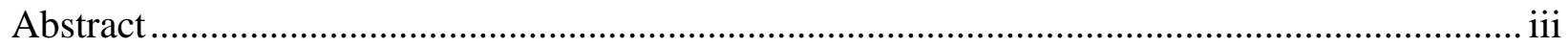

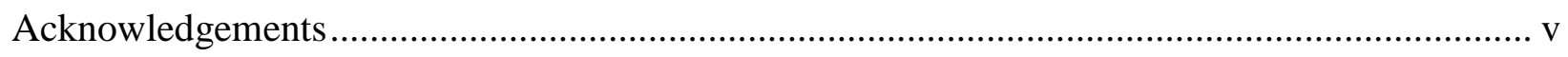

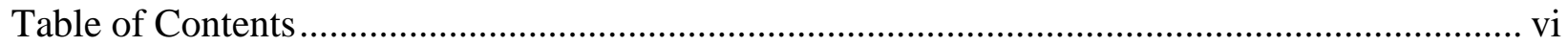

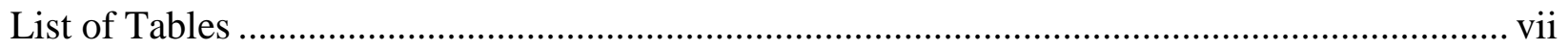

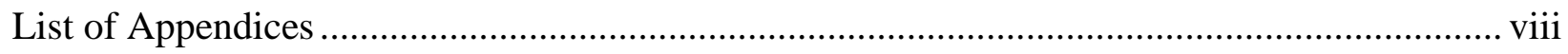

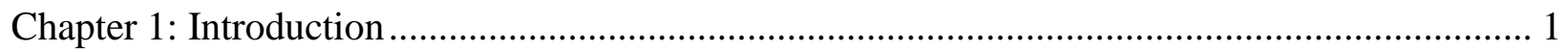

Chapter 2: An Experimental Examination of Heightened Emotional Intensity and Emotional

Reactivity in People with Generalized Anxiety Disorder ................................................ 6

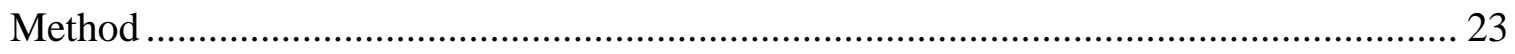

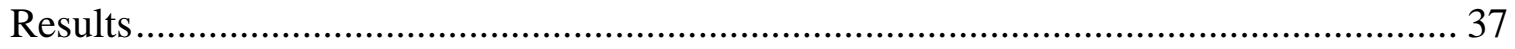

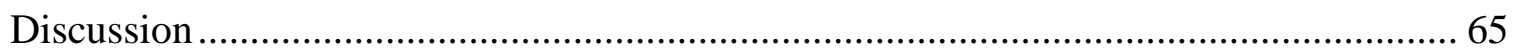

Chapter 3: An Experience Sampling Study of Emotional Experiences in the Daily Life of People

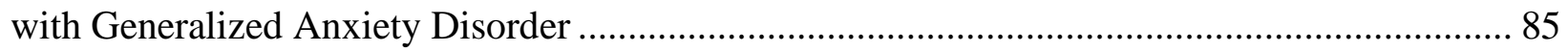

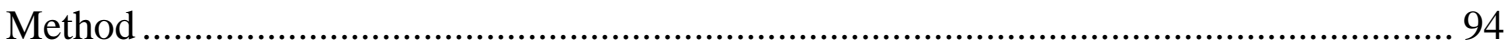

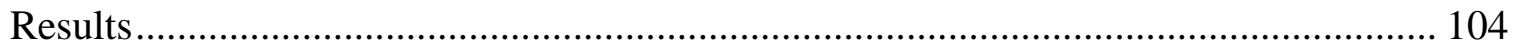

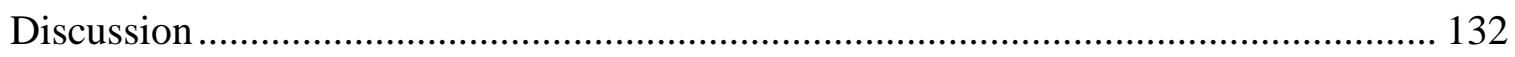

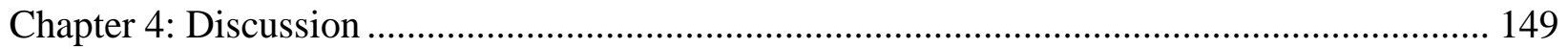

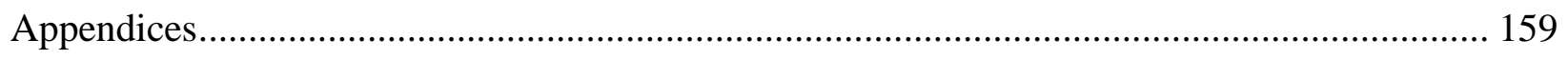

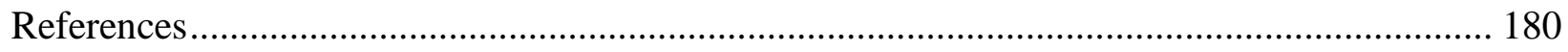




\section{List of Tables}

Table 1: Demographic Characteristics Separated by Group ................................................... 25

Table 2: Clinical Characteristics of the GAD, SAD and NCC groups ................................... 39

Table 3: Means and Standard Deviations of Emotion Induction Engagement Questions by Group

Table 4: Means and Standard Deviations on Self-Report Measures of Trait Emotional Intensity45

Table 5: Means and Standard Deviations on Self-Report Measures of Emotion Regulation....... 46

Table 6: Baseline Emotional Intensity as Assessed via Subjective Emotion Rating, RSA, and

SCR

Table 7: Emotional Reactivity to the Negative Emotion Induction........................................ 54

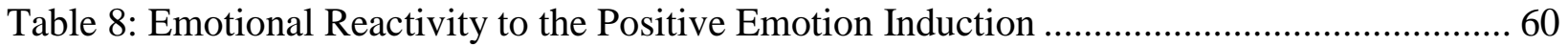

Table 9: Demographic Characteristics Separated by Group ................................................ 96

Table 10: Clinical Characteristics of the GAD and NCC groups ........................................ 105

Table 11: Means and Standard Deviations of Scores on Emotion-related Questionnaires

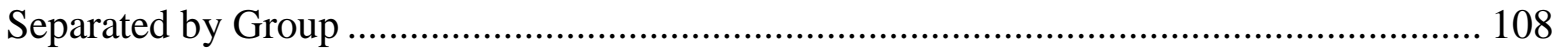

Table 12: Inertia of Negative and Positive Emotion as a Function of GAD Status.................. 116

Table 13: Emotional Response Following Worry as a Function of GAD Status ..................... 119

Table 14: Relations of Negative and Positive Emotion to Worry as a Function of GAD Status 122

Table 15: Percentage of All Reported Event Types Separated by Group............................... 124

Table 16: Percentage of All Reported Interactions During Events by Group ......................... 125

Table 17: Aggregated Mean Ratings for Events Separated by Group.................................... 126

Table 18: Examining Emotional Reactivity to a Negative Event as a Function of GAD Status 130

Table 19: Examining Emotional Reactivity to a Positive Event as a Function of GAD Status . 131 


\section{List of Appendices}

Appendix A: Mental Imagery Script Task ................................................................... 159

Appendix B: End of Study Follow-up Questions ........................................................ 163

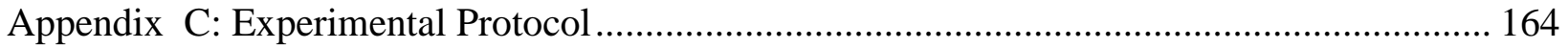

Appendix D: Informed Consent Agreement ................................................................ 165

Appendix E: Subjective Emotion Rating, Level of Worry and Current muscle tension/ bodily

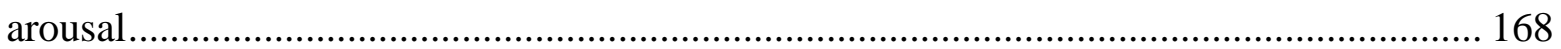

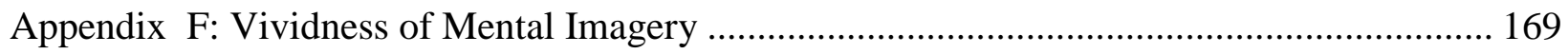

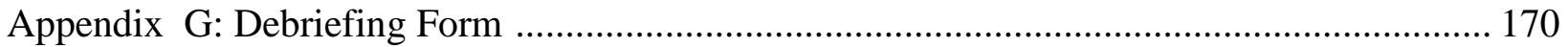

Appendix H: Informed Consent Agreement ............................................................ 172

Appendix I: Experience Sampling Method Protocol ...................................................... 176

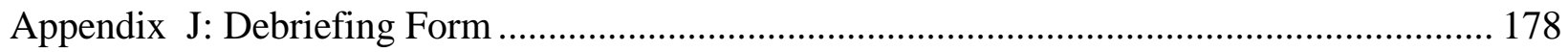




\section{Chapter 1: Introduction}

Generalized anxiety disorder (GAD) is characterized by excessive and uncontrollable worry and is associated with muscle tension, irritability, difficulties concentrating, sleep disturbances and fatigue (American Psychiatric Association, 2013). Interest in the relationship between difficulties with emotional experiences, emotion regulation, and anxiety disorders has

flourished over the past decade (Rodebaugh \& Heimberg, 2008). Theory and empirical evidence suggest that difficulties with emotions may play a significant role in the development and maintenance of anxiety psychopathology, and in particular, GAD (Mennin, Heimberg, Turk, \& Fresco, 2002).

Although the experience of emotions can be adaptive and can help people respond to demands in their external environment, emotions can also be maladaptive and lead people to engage in self-destructive thinking and behaviour (Parrott, 2001). As a result, the ability to successfully manage, or regulate one's emotional experiences is argued to have important mental health implications (Gross \& Muñoz, 1995). Emotion regulation is defined as "the process by which individuals influence which emotions they have, when they have them, and how they experience and express these emotions" (Gross, 1998b, p. 275). Difficulties in emotion and emotion regulation have been examined in many clinical populations including major depressive disorder, borderline personality disorder, eating disorders, and more recently in GAD.

\section{Emotion Regulation and GAD}

To better understand the role of emotion and emotion regulation in GAD, Mennin Heimberg, Turk, and Fresco (2005) proposed the emotion dysregulation model of GAD. This model is partially based on the avoidance theory of worry, which posits that people with GAD engage in worry to avoid experiencing negative emotions associated with aversive mental 
imagery (Borkovec, Alcaine, \& Behar, 2004). The emotion dysregulation model builds on the avoidance theory by providing a theoretical framework for examining why people with GAD experience emotions as aversive and engage in maladaptive strategies to avoid emotional experiences. The model proposes that people with GAD have difficulties with four interconnected aspects of emotional functioning: First, they experience emotions at a heightened intensity compared to people without GAD. Second, people with GAD have poor understanding of their emotions, which leads to difficulties with identifying, labeling, and describing emotions. Third, people with GAD react negatively to their emotions due to negative beliefs about the consequences of experiencing positive and negative emotions. Finally, people with GAD have difficulty managing their experience of negative emotions (Mennin et al., 2002; Mennin et al., 2005). Moreover, this model argues that GAD-type worry is a "cognitive control strategy" that functions to decrease attentional focus on one's emotional experience and in turn, reduce distress (Mennin et al., 2002).

In a preliminary test of the model, Mennin and colleagues (2005) compared people with high versus low symptoms of GAD on self-report questionnaires that assess the four components of the model. The four components were supported: people with heightened symptoms of GAD reported greater intensity of negative emotions, poorer understanding of their emotions, negative appraisals of their emotions, and greater perceived difficulty returning to their baseline mood following a negative mood induction compared to people without GAD.

Since Mennin and colleagues' (2002) seminal article proposing the emotion dysregulation model of GAD and Mennin et al.'s (2005) preliminary test of the model, several studies have been conducted to advance this line of research. In contrast to Mennin et al. (2005), however, subsequent tests of the model suggest that not all components may be supported or 
specifically relevant to people with GAD (cf. Behar, Di Marco, Hekler, Mohlman, \& Staples, 2009). For example, studies examining emotional awareness to determine whether people with GAD have poorer understanding of emotions, show that people with GAD actually have higher or equal levels of emotional awareness compared to people with social anxiety disorder (SAD), people with depression, and nonanxious controls (e.g., Decker, Turk, Hess, \& Murray, 2008; Mennin, Holaway, Fresco, Moore, \& Heimberg, 2007; Novick-Kline, Turk, Mennin, Hoyt, \& Gallagher, 2005).

Of note, in contrast to previous tests of the model that relied on self-report measures, Novick-Kline et al. (2005) and Decker et al. (2008) used alternative methods to assess emotional awareness. Novick-Klein et al. (2005) used an ability-based measure, the Levels of Emotional Awareness Scale (Lane, Quinlan, Schwartz, Walker, \& Zeitlin, 1990), which asks participants to describe emotional reactions to hypothetical situations. The authors reported that relative to nonanxious controls, coders rated the emotional reactions provided by people with GAD as containing more nuanced details, suggesting that they have a higher level of emotional awareness compared to nonanxious controls. Correspondingly, Decker et al. (2008) examined the emotions described in a daily diary and reported that people with GAD did not report greater difficulty discriminating among their emotions compared to people without GAD. These results suggest that people with GAD do not have deficits in their emotional awareness and therefore, this component of the model may not contribute to the understanding of why people with GAD have difficulties with emotion and emotion regulation. In addition, given the disagreement between ability-based measures and self-report measures, the difference may be better attributed to people's negative perceptions of their abilities. 
In addition, negative beliefs about the consequences of emotional experiences do not appear to be specific to people with GAD. Although people with GAD report experiencing greater fear of negative and positive emotions compared to nonanxious controls (Mennin et al., 2005), compared to people with SAD and people with depression, people with GAD do not report greater negative reactivity to emotional experiences (Mennin et al., 2007; Turk, Heimberg, Luterek, Mennin, \& Fresco, 2005). In fact, Mennin et al. (2007) reported that when controlling for comorbid disorders, negative reactivity to emotions appears to add to the prediction of depression and $\mathrm{SAD}$, but not GAD. In sum, these findings suggest that there may not be empirical support for all components of the emotion dysregulation model. Moreover, the findings highlight the benefit of using multimethod designs and including a clinical comparison groups to adequately test the model.

Two components of the model that have received empirical support and appear to be particularly relevant to people with GAD are heightened emotional intensity and difficulties with emotion management (i.e., emotion regulation). Across several studies and compared to nonanxious controls, people with SAD and people with depression, it has been consistently shown that people with GAD report heightened emotional intensity and difficulties with engaging in adaptive emotion regulation strategies (e.g., Mennin et al., 2007; Mennin, McLaughlin, \& Flanagan, 2009; Turk et al., 2005). However, these studies relied primarily on self-report questionnaires and thus, the use of alternative research methods, such as laboratory experiments and monitoring in daily life, would add to the existing literature on emotional intensity and emotion regulation in people with GAD. This dissertation comprises two independent studies examining emotion dysregulation in people with GAD to address these limitations. 
Study 1 (chapter 2) used a laboratory-based experimental research design to test the first tenet of the emotion dysregulation model, which proposes that people with GAD experience heightened emotional intensity. The aim of this study was to build on and extend previous tests of the model that relied primarily on trait self-report measures to assess heightened emotional intensity in people with GAD. In particular, the study examined momentary subjective and physiological emotional responses in people with GAD compared to people with social anxiety disorder (SAD) and people with no psychopathology at baseline (i.e., unmanipulated state) and following an emotion induction.

Study 2 (Chapter 3) was conducted to examine the intensity of emotional experiences of people with GAD in the context of their daily lives to determine if and how they may differ from people with no psychopathology. In addition, the study investigated how worry, a maladaptive emotion regulation strategy, leads to changes in people's momentary emotional experiences, and the extent to which this differs for people with GAD compared to people with no psychopathology. Finally, the study examined the extent to which people with GAD exhibit heightened emotional reactivity to negative and positive events in daily life compared to people with no psychopathology. 


\section{Chapter 2: An Experimental Examination of Heightened Emotional Intensity and Emotional Reactivity in People with Generalized Anxiety Disorder}

The emotion dysregulation model posits that people with generalized anxiety disorder (GAD) have dysfunction in the process of emotion generation such that they experience emotions at a heightened intensity compared to people without GAD (Mennin, 2002; Mennin et al., 2005). Mennin et al. (2005) describe heightened emotional intensity as "frequently experiencing negative affect strongly" and having "emotional reactions that occur intensely, easily and quickly" (p. 286). Although preliminary findings support this assumption, there is still much that remains unknown about the emotion generative process and the construct of heightened emotional intensity in people with GAD. Therefore, Fresco, Mennin, Heimberg, and Ritter (2013) argued that there is a need for more "rigorous" experimental research to better understand the experience of heightened emotional intensity in people with GAD.

\section{Investigations of Emotional Intensity in GAD}

Extant studies provide preliminary support that the emotional experiences of people with GAD appear to differ from those of people with SAD, people with depression, and nonclincal controls. Mennin et al. (2005) found that people with elevated symptoms of GAD reported greater emotional strength and greater expression of negative emotions compared to people low in symptoms of GAD on the Berkeley Expressivity Questionnaire (BEQ; Gross \& John, 1997), but that the two groups did not differ on their self-perceived expression of positive emotions (Mennin et al., 2005). In a follow-up study, Turk et al. (2005) compared people with elevated symptoms of GAD to people with elevated symptoms of SAD and nonanxious controls on the BEQ. Consistent with Mennin et al. (2005), people with GAD reported stronger emotional experiences, but equal levels of expressivity of positive emotions compared to the nonanxious 
controls. People with GAD also reported stronger emotional experiences on the BEQ compared to people with SAD. The study did not replicate Mennin's finding that people with GAD report greater expressivity of negative emotions, however, as there were no differences found between the three groups on expressivity of negative emotions. To note, compared to people with GAD and nonanxious controls, people with SAD reported lower expressivity of positive emotions.

A limitation of the above-mentioned studies is the reliance on the BEQ, as it does not explicitly assess the intensity of emotional experiences. Rather, the BEQ is a measure of people's level of agreement with statements that describe a greater expression of positive (e.g., "When I'm happy, my feelings show") and negative emotions (e.g., "I sometimes cry during sad movies"), as well as the overall strength of their emotional experiences (e.g., "I have strong emotions"; Gross \& John, 1997). In sum, because the BEQ does not provide a pure assessment of emotional intensity, additional evidence is required to demonstrate whether people with GAD indeed experience emotions at a heightened intensity (Bachorowski \& Braaten, 1994).

In an effort to improve upon the limitations of the BEQ, Mennin and colleagues (2009) conducted a follow-up study using the Affect Intensity Measure (AIM; Larsen \& Deiner, 1987) as a measure of emotional intensity. The AIM assesses how frequently (i.e., never to almost always) people experience intense emotions (e.g., "Sad movies touch me deeply"). On the AIM, participants with GAD reported experiencing intense negative emotions at a greater frequency compared to people with SAD and people without GAD or SAD. The study did not examine frequency of intense positive emotions. Given that the AIM assesses how frequently people report experiencing intense emotions, it also does not provide an explicit assessment of the intensity of the emotion itself (Bachorowski \& Braaten, 1994). Given the limitations of the BEQ and the AIM as described by Bachorowski and Braaten (1994), the aforementioned studies may 
not provide an accurate picture of the extent to which people with GAD experience emotions at a greater intensity compared to people with another psychological disorder or to people without GAD.

To gain a better understanding of how to best define and measure emotional intensity in people with GAD, the extant literature on emotion dysregulation in other psychological disorders may be informative. Researchers examining emotional intensity in people with borderline personality disorder suggest that rather than viewing heightened emotional intensity as a single construct, it is necessary to separate baseline emotional intensity from emotional reactivity (Kuo \& Linehan, 2009). Baseline emotional intensity is defined as people's baseline emotionality that is independent of the presence of an emotional stimulus (Jacob et al., 1999). Emotional reactivity is defined as the emotional response following exposure to an emotionally evocative stimulus (e.g., Kuo \& Linehan, 2009). Therefore, additional research examining the construct of heightened emotional intensity in people with GAD may benefit from separate investigations of their baseline emotional state and level of emotional reactivity.

Kuo and Linehan (2009) highlight that studies examining emotion dysregulation based on self-report measures are limited because the assessment of baseline emotional intensity and emotional reactivity are often confounded within self-report questionnaires. For example, the positive affect subscale of the AIM contains items that describe the intensity of people's emotions (e.g., "When I feel happy it is a strong type of exuberance") as well as their degree of reactivity to an emotional event (e.g., "When someone compliments me, I get so happy I could 'burst"'). Memory biases may also influence findings based primarily on trait self-report measures given that they rely on participants' retrospective reports of past emotional experiences (Stone et al., 1998). Therefore, alternative methods including real-time, momentary assessments 
of people's emotional intensity in their baseline (i.e., unmanipulated) state and immediately following an emotional stimulus are necessary (e.g., Kuo \& Linehan, 2009).

A small number of studies have examined momentary differences in baseline emotional intensity and emotional reactivity in people with GAD compared to people without GAD:

Baseline emotional intensity. As a part of a larger study, Llera and Newman (2014) compared people with heightened symptoms of GAD to nonanxious controls on their responses to a measure assessing their current experience of 8 distinct emotions (i.e., amusement, anger, contentment, disgust, fear, happiness, sadness and tension) while in an unmanipulated, baseline state. People with elevated symptoms of GAD reported greater sadness, lower contentment and lower happiness compared to nonanxious controls. There were no differences reported on the other emotions. Therefore, at an unmanipulated baseline state, people with heightened symptoms of GAD report differences in their intensity of specific emotions compared to nonanxious controls.

The finding that people with GAD report greater intensity of baseline sadness provides support to previous findings that people with GAD report greater intensity of negative emotions compared to people without GAD on trait measures of emotional expressivity and intensity (e.g., Mennin et al., 2005; Mennin et al., 2009). In contrast, the finding that people with GAD reported lower intensity of positive emotions differed from previous studies that indicated that there were no differences between people with and without GAD on a trait measure of positive emotional expressivity (e.g., Mennin et al., 2005). In the study of emotional intensity in people with GAD, only a few studies have explicitly assessed positive emotional experiences. Therefore, it is important to continue to investigate the extent to which people with GAD report lower intensity of positive emotions compared to people without GAD. 
Moreover, Watson, Clark, and Stasik (2011) reported discrepancies in the association between symptoms of GAD and trait versus state measures of positive affect. That is, on a trait measure of affect, they found that symptoms of GAD were not associated with positive affect ( $r$ $=-.13, p>.05)$, whereas on a state measure of affect, symptoms of GAD were negatively correlated with positive affect $(r=-.40, p<.05$; Watson et al., 2011). Thus, these findings further highlight discrepancies between trait and state measures of emotional experiences. Given that the majority of research on emotional intensity has relied on findings from trait self-report measures, it is important to further examine emotional intensity in people with GAD using alternative assessment methods, such as measures that examine emotions "in the moment" as opposed to trait self-report measures.

Emotional reactivity. A small number of studies have employed laboratory tasks to examine the intensity of people's emotional reactions following an emotional stimulus. In a study examining emotional reactivity to a music clip, Mennin et al. (2005) reported that people with heightened symptoms of GAD experienced greater subjective distress following a negative mood induction compared to nonanxious controls. Mennin et al. also reported that people with higher symptoms of GAD experienced greater positive emotional reactivity to a neutral emotion induction compared to the nonanxious controls. That is, participants with GAD described the neutral music clip as calming. The authors suggest that this finding may indicate that people with GAD may also have greater positive emotion reactivity, not just negative emotional reactivity. However, this finding is inconsistent with research suggesting that people with GAD report greater fear of the consequences of experiencing positive emotions compared to nonanxious participants (Mennin et al., 2005) and have a greater tendency to dampen positive emotions (Eisner, Johnson, \& Carvers, 2009). Further examination of emotional reactivity to a positive 
emotion induction would help to clarify the extent to which people with GAD exhibit adaptive or maladaptive emotional responses to positive stimuli.

Other laboratory experiments examining emotional reactivity in people with GAD have focused primarily on the role that worry has in influencing the intensity of people's reactivity to an emotional stimulus (e.g., Llera \& Newman, 2010; 2014; McLaughlin, Mennin, \& Farach, 2007). For example, prior to viewing an emotionally evocative film clip, Llera and Newman (2014) instructed participants to engage in a worry induction (e.g., "Think about your most worrisome topic and worry about it as intensely as you can"). Therefore, conclusions regarding emotional reactivity based on studies where the participants were induced to worry prior to the emotion induction are limited because they do not examine people's emotional reactivity from a baseline, unmanipulated state. In sum, additional research is needed to clarify whether people with GAD experience heightened emotional reactivity to negative and/or positive emotional stimuli compared to people without GAD.

\section{Physiological Response as a Measure of Emotional Intensity}

Emotions comprise multiple response components, including subjective responses and physiological responses (Gross \& Thompson, 2007; Lang, Bradley, \& Cuthbert, 1998). Initial studies testing the construct of heightened emotional intensity relied primarily on people's subjective responses (e.g., Mennin et al., 2005; Mennin et al., 2009); therefore, including assessments of physiological responses provides an objective measure of people's emotional reactivity. Particularly relevant to the experience of emotion are physiological responses that correspond to the sympathetic and parasympathetic nervous system, including skin conductance and respiratory sinus arrhythmia (RSA), respectively (Bradley \& Lang, 2007).

Skin conductance is a measure of electrical properties of the skin that occur in response 
to sweat (Dawson, Schell, \& Filion, 2007). Baseline skin conductance level (SCL) and nonspecific skin conductance responses (NS-SCR) are useful measures of sympathetic emotional arousal given that both are unaffected by the parasympathetic system. Therefore, NS-SCR can be used as a measure of emotional activation, even if there are limited changes to cardiac measures (cf. Salters-Pedneault, Gentes, \& Roemer, 2007). In addition, regardless of emotional valence, the amplitude of NS-SCRs increase as arousal increases (Bradley \& Lang, 2007) and therefore, NS-SCR is a reliable assessment of emotional reactivity in response to positive and negative stimuli.

RSA, or high-frequency heart rate variability (HF-HRV), is a measure of fluctuations in people's heart rate as linked to their breathing rate, and is an index of vagal tone (Berntson, Quigley, \& Lozano, 2007). At rest, high RSA is associated with slower heart rate, whereas low RSA is suggestive of stable high heart rate (Levy, 1984; Porges, 1991); thus, higher resting RSA is adaptive. In terms of emotional reactivity, when people experience a stressor or an emotional stimulus, the "vagal brake" is withdrawn (i.e., vagal withdrawal), resulting in an increased heart rate (i.e., decreased RSA). An increase in heart rate following a stressor is an adaptive process as it allows the individual to meet the demands of their environment. In addition, a quicker return to baseline RSA following an emotional stimulus is indicative of a flexible and adaptive parasympathetic system (Porges, 2007).

Both the sympathetic and parasympathetic systems are important systems to consider when examining people's emotional experiences, as dysfunction in one or both of the systems may provide empirical support for heighted emotional intensity and emotional reactivity in people with GAD. Previous studies of physiological measures in people with GAD have shown mixed findings on their baseline autonomic arousal compared to nonanxious controls. On the one 
hand, people with GAD are found to have low RSA in laboratory (Thayer, Friedman, \& Borkovec, 1996) and in ambulatory (Hoehn-Saric, McLeod, Funderburk, \& Kowalski, 2004) studies compared to nonanxious controls, and greater SCRs compared to individuals with depression or obsessive-compulsive disorder (Pruneti, Lento, Fante, Carrozzo, \& Fontana, 2010). On the other hand, other studies report no baseline differences between people with GAD and nonanxious controls in RSA (Fisher \& Newman, 2013), HF-HRV (Llera \& Newman, 2010) or SCR (Fisher, Granger, \& Newman, 2010; Llera \& Newman, 2014). Therefore, the extent to which people with GAD experience autonomic inflexibility, at baseline, is unclear. Moreover, the mixed findings suggest that people with GAD may display variability in their baseline sympathetic and parasympathetic responses.

In terms of understanding emotional reactivity from a physiological perspective, in nonclinical samples, anxiety- (e.g., threat of shock, speech preparation) or fear- (e.g., exposure to frightening or aversive film clips) evoking stimuli are often associated with sympathetic activation (i.e., increased SCRs) and vagal withdrawal (i.e., decreased RSA; Kreibig, 2010). This does not appear to be the case for people with GAD, however, as they often have rigid autonomic responses (i.e., lower physiological flexibility) following exposure to negative stimuli. Specifically, people with GAD exhibit low HF-HRV and limited SCR reactivity in response to perceived stressors (Fisher et al., 2010; Hoehn-Saric \& McLeod, 2000). Moreover, Lyonfields, Borkovec, and Thayer (1995) reported that people with high symptoms of GAD did not exhibit changes in RSA after viewing aversive images, whereas nonanxious controls showed a decrease in RSA. In sum, these findings suggest that people with GAD may have less physiological reactivity to emotional stimuli, and exhibit chronically low vagal tone, which is suggestive of a stable high heart rate and lower physiological flexibility. 
In terms of physiological response, positive emotions are also associated with elevated autonomic arousal (Shiota, Newfeld, Yeung, Moser, \& Perea, 2011), including increased sympathetic and parasympathetic activity (Kreibig, 2010; Lang, Greenwald, Bradley, Hamm, 1993). Unlike the physiological arousal associated with the experience of negative emotions, however, the physiological arousal associated with positive emotions may be associated with beneficial health outcomes. That is, in a sample of healthy individuals, increased positive emotion (i.e., inducing appreciation) was associated with increased HF-HRV (McCraty, Atkinson, Tiller, Rein, \& Watkins, 1995). Fredrickson (1998) argues that positive affective experiences may be a "stress buffer," and may function to influence the intensity and recovery from stressful events.

The only known study to examine physiological response following a positive emotion induction was the experimental study conducted by Llera and Newman (2014). The authors reported that regardless of the manipulation that people engaged in (i.e., worry, relaxation or neutral induction), there was no difference between people with GAD and nonanxious controls in their NS-SCRs following a humourous film clip. That is, both groups showed an increase in NSSCRs following the exposure suggesting increased sympathetic arousal. This finding suggests that people with GAD may experience similar emotional reactivity as evidenced by their physiological response to positive emotion inductions compared to people without GAD.

\section{Present Study}

Over the past decade, several cross-sectional, correlation studies and laboratory experiments have been conducted to better understand emotion generation in people with GAD. The present study built upon this knowledge and extended it in several ways, including the use of a GAD sample, a clinical comparison group, and idiographic emotion inductions. 
Clinical sample. The study of emotion generation in GAD would benefit from examining baseline emotional intensity and emotional reactivity in a clinical sample of people who report symptoms meeting diagnostic criteria for DSM-5 GAD (American Psychiatric Association, 2013). To date, studies of emotional experiences in GAD have mainly used nonclinical student samples and analogue community samples classified as having GAD by a diagnostic self-report measure (e.g., McLaughlin et al., 2007; Mennin et al., 2005; Mennin et al., 2009). A limitation of student samples is that they tend to be higher functioning than clinical samples (cf. Mennin et al., 2009). Therefore, it is prudent to extend these results by reevaluating the intensity of emotional experiences in a clinical sample of people with GAD.

Clinical comparison group: Social anxiety disorder (SAD). The inclusion of a clinical comparison group assists in determining the extent to which heightened emotional intensity, in the absence or presence of an emotional stimulus, is specific to people with GAD compared to people with a different psychological disorder. People with SAD were selected to be consistent with the preliminary tests of the emotion dysregulation model, which compared analogue GAD and SAD samples (e.g., Mennin et al., 2009; Turk et al., 2005).

Baseline emotional intensity. Studies comparing nonclinical samples of people with elevated symptoms of GAD to people with elevated symptoms of SAD have provided preliminary support that the two groups experience differences in their self-reported levels of emotional intensity (e.g., Mennin et al., 2007; Turk et al., 2005;). In comparison to people with SAD, people with GAD are found to report greater expressivity and frequency of intense negative emotions on trait self-report measures (Mennin et al., 2007; Mennin et al., 2009; Turk et al., 2005). In addition, heightened expressivity of negative emotions is positively predictive of GAD, but negatively predictive of SAD (Mennin et al., 2007). Thus, GAD and SAD appear to be 
associated with different degrees of emotional expressivity and/or difficulties with emotion regulation.

The present study also extends previous examinations by comparing people with GAD and people with SAD on their baseline sympathetic and parasympathetic responses. There is limited research directly comparing people with GAD and people with SAD on autonomic responses and of the known studies, the findings appear to be mixed. For example, in a study comparing parasympathetic response, Pittig, Arch, Lam, and Craske (2013) found that of participants with a diagnosis of a DSM-IV anxiety disorder, those with GAD or SAD had lower baseline HF-HRV compared to nonanxious controls, which suggests that both clinical groups exhibit parasympathetic inflexibility. Inconsistent with this, however, Kuo and Linehan (2009) reported no differences in resting RSA in people with SAD and people with no psychopathology, which does not support the inflexibility hypothesis.

Regarding the sympathetic nervous system, no known studies have compared people with GAD to people with SAD on their baseline sympathetic arousal as assessed by NS-SCRs. Research examining the autonomic responses of people with SAD compared to people with no psychopathology has shown that people with SAD have a greater frequency of NS-SCRs during a resting baseline state compared to nonanxious controls $(d=.82$; Kuo \& Linehan, 2009). The present study will add to the existing literature with the aim of better understanding the baseline physiological experiences of people with GAD directly in comparison to people with SAD, as well as to people with no psychopathology.

Emotional reactivity. Few studies have directly compared the emotional response of people with GAD to that of people with SAD following an emotional provocation. In one study, Macatee and Cougle (2013) examined the extent to which participants' scores on the Penn State 
Worry Questionnaire (PSWQ) and scores on Fear of Negative Evaluation (FNE) were associated with degree of reactivity to emotional stimuli (i.e., video clips) in a sample of undergraduate students. Emotional reactivity was assessed using a self-report rating of level of peak fear, disgust, sadness and anger experienced during the video clip $(1=$ none at all to $6=$ extreme $)$. The authors reported that elevated scores on the PSWQ were predictive of greater negative emotional reactivity to negative mood inducing film clips over and above elevated scores on the FNE. The results suggest that elevated worry may have a stronger association with heightened emotional reactivity to negative stimuli compared to a fear of negative evaluation. In addition, Kuo and Linehan (2009) found that people with SAD did not differ from people with no psychopathology on their reported emotions following negative mood inductions (i.e., video clips and idiographic scripts) that evoked sadness, fear, or anger, $d=.53, .38$, and .38 respectively. Thus, the present study will clarify whether heightened emotional reactivity to negative stimuli is specific to people with GAD compared to people with SAD.

No known studies have compared people with GAD to people with SAD in their reported positive, or negative, emotions following a positive emotion induction. Similar to people with GAD, on trait self-report questionnaires of emotional intensity, people with SAD report greater negative reactivity to positive emotions (Turk et al., 2005). Unlike people with GAD, people with SAD also report lower expressivity of positive emotions compared to nonanxious controls (Turk et al., 2005). Therefore, the present study extended the existing research on emotional reactivity by examining whether people with GAD differ from those with $\mathrm{SAD}$ and people with no psychopathology in their subjective response to a positive emotional induction.

Finally, no known studies have examined the extent to which people with GAD differ from people with SAD in their physiological reactivity to an emotional stimulus. Research 
examining how people with SAD react to negative stimuli has reported mixed findings. In terms of sympathetic responding, Moscovitch, Suvak and Hofmann (2010) reported that relative to people without SAD, people with SAD displayed an increase in SCRs following a speech task (i.e., negative mood induction). However, Kuo and Linehan (2009) reported that people with SAD did not show greater SCRs following video and audio emotion inductions of fear, sadness, or anger compared to people with no psychopathology, $d=.11, .02$, and .11 respectively. Moreover, previous findings suggest that people with generalized SAD and people with no psychopathology have comparable parasympathetic responses to emotional stimuli, including RSA (Kuo \& Linehan, 2009) and heart rate (Hofmann, Moscovitch, \& Kim, 2006) reactivity following a mood induction.

In sum, the present study adds to the literature by examining whether people with GAD differ from people with SAD on their subjective and physiological emotional reactivity to a negative and positive emotion induction. Moreover, the study helps to clarify whether the responses to negative and positive events differ for people with GAD compared to people with SAD. For example, the evidence suggests that people with GAD may experience elevated positive emotions following a positive emotion induction, but people with SAD may not experience elevated positive emotion. A better understanding of how people respond emotionally and the extent to which this differs for people with GAD from those with SAD may help to distinguish the type of emotional response that is common to each anxiety disorder.

Idiographic emotion induction. Studies experimentally inducing emotion in people with GAD have used standardized stimuli, such as film and music clips (e.g., Macatee \& Cougle, 2013; Mennin et al., 2005). Although there are advantages to standardized stimuli, such as greater control over the stimuli presented, there may be benefits to idiographic stimuli that 
outweigh the use of standardized stimuli. Huppert and Foa (2004) argue that the activation of fear occurs when people encounter stimuli that are representative of their fear structures, which suggests that the closer the match between an evoking experience and a person's fear structure, the greater the level of emotional activation. Alternatively, when an individual is presented with standardized stimuli, the mental imagery evoked may be less threatening given its lower ecological validity, and in turn, the associated emotional experience would be rated as being lower in intensity (Orr, Pitman, Lasko, \& Herz, 1993). Indeed, compared to standardized scripts, personalized stimuli are associated with greater self-reported vividness of mental imagery (Cook, Melamed, Cuthbert, McNeil and Lang, 1988), greater physiological response (i.e., elevated heart rate, SCRs, and facial electromyography activity; Miller, Patrick, \& Levenston, 2002) and greater subjective experiences of anxiety and tension (Velasco \& Bond, 1998).

To my knowledge, there are no known studies that have compared the use of standardized and idiographic stimuli to examine emotional reactivity in people with GAD. Findings from studies examining attentional and memory biases in people with GAD, however, suggests that the use of personal threatening information triggers greater distress in people with GAD compared to the use of standardized threatening information (Coles, Turk, \& Heimberg, 2007; Mathews \& Klug, 1993). One reason may be due to the significant interpersonal variability in the types of concerns that evoke distress in people with GAD (e.g., finance, health, safety, future, etc.), and therefore, if the standardized threat information presented is irrelevant to the individual, it may not result in a significant emotional response. Thus, having people with GAD engage in an idiographic emotion induction whereby they imagine a personal event occurring (e.g., a worst-case scenario) would build upon previous studies that have used standardized emotion inductions to evoke emotional responses in people with GAD. 


\section{Research Questions and Hypotheses}

The main goal of the research was to better understand the emotion generative process in people with GAD compared to people with SAD and to people with no psychological disorders (i.e., nonclinical controls; NCC). A secondary goal was to clarify the extent to which heightened emotional intensity in people with GAD is due to their baseline emotional experiences or to their emotional reactivity. That is, if people with GAD report greater intensity of emotions at baseline and following an emotional stimulus (while statistically controlling for their baseline intensity) compared to people with no psychopathology and people with SAD, this would suggest that people with GAD have deficits in both components of the emotion generative process.

In the study, baseline emotional intensity was operationalized as participants' baseline emotional experience independent of intentional exposure to an emotional stimulus (Jacob et al., 1999). Emotional reactivity was operationalized as the mean difference between emotional intensity, as assessed by participants' subjective and physiological responses, prior to and following the idiographic emotion inductions (Kuo \& Linehan, 2009).

Objective 1: Baseline emotional intensity. Based on theory and previous empirical findings, it was hypothesized that people with GAD would report heightened intensity of their baseline negative emotions compared to people with SAD and people with no psychopathology and report heightened intensity of positive emotions compared to people with SAD, but report lower intensity of positive emotions compared to people with no psychopathology. Based on the existing, although mixed findings on baseline physiology, it was hypothesized that people with GAD would display lower parasympathetic arousal (i.e., lower baseline RSA) compared to people with no psychopathology, but would not differ compared to people with SAD. Finally, 
regarding baseline sympathetic arousal, it was hypothesized that the three groups would not differ in the frequency of the NS-SCRs.

Objective 2: Emotional reactivity. The present study also examined the degree to which people with GAD report heightened reactivity to a negative and/or positive stimulus compared to people with SAD and people with no psychopathology.

Objective 2a. Negative emotion induction. Based on theory, it was hypothesized that following a negative emotion induction, people with GAD would report a greater increase in their subjective intensity of negative emotions compared to people with SAD and people with no psychopathology. It was also hypothesized that the negative emotion induction would be associated with a decrease in the intensity of positive emotions, however, it was unknown if this would differ across the groups. Regarding physiological response to the negative emotion induction, it was expected that in comparison to people with no psychopathology and people with SAD, people with GAD would exhibit greater physiological inflexibility, as evidenced by a lesser degree of change in RSA and frequency of NS-SCRs.

Objective 2b: Positive emotion induction. Based on the mixed findings in the literature regarding the degree of positive emotional reactivity in people with GAD, it was hypothesized that people with GAD would report a significant increase in positive emotion from their baseline, unmanipulated state to following the positive emotion induction; however, it was unknown whether the degree of change would be comparable to people with no psychopathology. Based on empirical evidence that SAD is often associated with suppressed expression of positive emotions (Kashdan \& Steger, 2006), it was hypothesized that following the positive emotion induction, people with GAD would report a greater increase in the intensity of positive emotions from their baseline, unmanipulated state compared to people with SAD. It was also hypothesized 
that the positive emotion induction would be associated with a decrease in the intensity of negative emotions, however, it was unknown if this would differ across the three groups.

Related to physiological response, it was hypothesized that people with GAD and people with no psychopathology would show increased sympathetic arousal (i.e., greater NS-SCRs) following the positive emotion induction. Although people with GAD have not been compared to people with SAD following a positive emotion induction, based on theory it was hypothesized that people with GAD would exhibit greater sympathetic response to the positive emotion induction compared to people with SAD. No known studies have examined parasympathetic responses to a positive emotion induction in people with GAD, therefore, it was unknown whether people with GAD would differ from people with SAD and people with no psychopathology on RSA reactivity following a positive emotion induction. Given that this was an exploratory question, no a priori hypothesis was advanced. 


\section{Method}

\section{Participants}

Individuals with GAD, SAD or no psychological disorder (nonclinical control; NCC) were recruited from the community via posters and online advertisements. Individuals who inquired about the study were invited to complete a telephone interview to determine eligibility. During the telephone screen, individuals who were 18 years and older were administered the Mini International Neuropsychiatric Interview-7 (MINI 7.0; Sheehan, 2014). At the completion of the telephone screen, individuals were invited to participate in the study if they: 1) endorsed symptoms for DSM-5-defined GAD; 2) reported symptoms for DSM-5-defined SAD (generalized); or 3) did not report symptoms that met the diagnostic criteria for a current DSM-5defined psychological disorder. To maintain discrete groups, individuals with comorbid diagnoses of GAD or SAD were not eligible to participate. Individuals who had comorbid diagnoses were eligible to participate in the study on condition that the symptoms of the comorbid disorder were identified as being less severe than the principal disorder (i.e., GAD or SAD).

People were excluded if they endorsed: current or past mania, hypomania, or psychosis; diagnostic criteria for alcohol use disorder or substance use disorder in the past 3 months (people were classified as "in early remission" if criteria were not met between 3 and 12 months); epilepsy or seizure disorder; heart disease; and/or asthma; clinically significant suicidal ideation; and/or colourblindness (the vanilla baseline task required the identification of different colours). In addition, people were excluded if they were currently taking tricyclic antidepressants, SSRIs, tranquilizers, antihistamines, beta blockers, or benzodiazepines, as these medications alter vagal and electrodermal activity (e.g., Adinoff, Mefford, Waxman, \& Linnoila, 1992; Tucker et al., 
2000; Yeragani et al., 1992).

A total of 197 participants were screened for the study, 86 met eligibility criteria, 12 participants did not start the study, and 9 did not complete all study components. The final sample comprised 65 participants; specifically, 22 GAD, 23 SAD, and 20 NCC participants.

Participant demographics. Of the 65 participants, $67.7 \%$ were female and $32.3 \%$ were male. Age of participants ranged between 18 and 64 years, with a mean age of 30.50 years $(S D=$ 12.20). The sample was ethnically diverse, with individuals self-identifying as: White (55.4\%), East Asian (10.8\%), Mixed (7.6\%), Latin American (6.2\%), Other (6.2\%), Black (4.6\%), South Asian (4.6\%) or South East Asian (4.6\%).

Demographic information for each group (GAD, SAD, and NCC) is presented in Table 1. There were significant differences found in gender across the groups, with the NCC group having significantly more males than the GAD group and the SAD group. There were no other significant differences in demographic characteristics across the groups.

In terms of diagnostic comorbidities based on the MINI 7.0 assessment, in the GAD group, $6(27.3 \%)$ participants had comorbid major depressive disorder (MDD) and 1 participant (4.5\%) had panic disorder. In the SAD group, 4 (17.4\%) participants had comorbid MDD, 1 participant (4.3\%) had comorbid panic disorder and 1 (4.3\%) participant had comorbid bulimia. The rates of comorbidity did not differ between the GAD group and SAD group. 
Table 1

Demographic Characteristics Separated by Group

\begin{tabular}{|c|c|c|c|c|}
\hline & $\begin{array}{l}\text { GAD } \\
n=22 \\
\end{array}$ & $\begin{array}{c}\text { SAD } \\
n=23\end{array}$ & $\begin{array}{c}\mathrm{NCC} \\
n=20 \\
\end{array}$ & $F$ or $\chi^{2}$ \\
\hline Age in years $-M(S D)^{a}$ & $28.14(11.01)$ & $30.13(12.29)$ & $33.68(13.32)$ & 1.07 \\
\hline Sex - Frequency $(\%)$ & & & & $6.81 *$ \\
\hline Female & $17(77.3)$ & $18(78.3)$ & $9(45)$ & \\
\hline Male & $5(22.7)$ & $5(21.7)$ & $11(55)$ & \\
\hline \multicolumn{2}{|l|}{ Race/Ethnicity - Frequency } & & & 13.24 \\
\hline Black & $1(4.5)$ & $0(0.0)$ & $2(10.0)$ & \\
\hline East Asian & $1(4.5)$ & $4(17.4)$ & $2(10.0)$ & \\
\hline Latin American & $2(9.1)$ & $2(8.7)$ & $0(0.0)$ & \\
\hline South Asian & $0(0.0)$ & $1(4.3)$ & $2(10.0)$ & \\
\hline South East Asian & $1(4.5)$ & $2(8.7)$ & $0(0.0)$ & \\
\hline White & $15(68.2)$ & $11(47.8)$ & $10(50.0)$ & \\
\hline Mixed & $1(4.5)$ & $1(4.3)$ & $3(15.0)$ & \\
\hline Other & $1(4.5)$ & $2(8.7)$ & $1(5.0)$ & \\
\hline \multicolumn{2}{|l|}{ Marital Status- Frequency } & & & 0.64 \\
\hline Single & $17(77.3)$ & $19(82.6)$ & $15(75.0)$ & \\
\hline Married/ Common-law & $3(13.6)$ & $3(13.0)$ & $3(15.0)$ & \\
\hline Divorced/widowed & $2(9.1)$ & $1(4.4)$ & $2(10.0)$ & \\
\hline \multicolumn{2}{|c|}{ Employment Status - Frequency (\%) } & & & 4.82 \\
\hline $\begin{array}{l}\text { Not Working } \\
\text { (including student) }\end{array}$ & $10(45.5)$ & $8(34.8)$ & $5(26.3)$ & \\
\hline Working part time & $7(31.8)$ & $12(52.2)$ & $7(36.8)$ & \\
\hline Working full time & $5(22.7)$ & $3(13.0)$ & $7(36.8)$ & \\
\hline $\mathrm{BMI}-\mathrm{M}(\mathrm{SD})$ & $23.89(2.64)$ & $23.05(3.71)$ & $24.51(3.25)$ & 0.99 \\
\hline
\end{tabular}

Note. $\mathrm{BMI}=$ Body Mass Index.

$* p<.05$

${ }^{\mathrm{a}}$ One person in the NCC group chose not to disclose his or her age. 


\section{Measures}

Telephone screen. The MINI International Neuropsychiatric Interview-7 (MINI 7.0; Sheehan, 2014) is a semistructured diagnostic interview that assesses for DSM-5-defined disorders (American Psychiatric Association, 2013). The MINI was administered via the telephone to assess whether DSM-5 diagnostic criteria were met for GAD, SAD, or no psychological disorder.

\section{Trait self-report measures of anxiety and depression symptoms. The Penn State} Worry Questionnaire (PSWQ; Meyer, Miller, Metzger, \& Borkovec, 1990) includes 16 items that assess the perceived excessiveness and uncontrollability of worrying (e.g., "Once I start worrying I can't stop"). Items are rated on a 5-point scale $(1=$ not at all typical of me to $5=$ very typical of me) and the ratings are summed for a total score, with higher scores indicating greater worry. The PSWQ has demonstrated high internal consistency $(\alpha=.86$ to .91; Dear et al., 2011) and good test-retest reliability $(r=.74$ to $r=.92$; Startup \& Erickson, 2006). The PSWQ demonstrated excellent internal consistency in the present study $(\alpha=.96)$.

The Social Phobia Inventory (SPIN; Connor et al., 2000) is a 17-item measure of severity of social anxiety disorder (e.g., "Talking to strangers scares me," "I would do anything to avoid being criticized."). Each item is rated on a 5-point likert scale $(0=$ not at all to $4=$ extremely). The SPIN has excellent internal consistency $(\alpha=.92$ total score) and good test-retest reliability $(r$ $=.86, p<.001 ;$ Antony, Coons, McCabe, Ashbaugh, \& Swinson, 2006). A high level of internal consistency was found in the present study $(\alpha=.96)$.

The State-Trait Inventory for Cognitive and Somatic Anxiety (STICSA; Ree, French, MacLeod, \& Locke, 2008) consists of two 21-item measures that assess cognitive and somatic anxiety. The STICSA-Trait assesses how people feel generally, while the STICSA-State 
measures how people feel in the moment. Sample items include: "My heart beats fast," and "I think the worst will happen." Items are rated on a 4-point scale $(1=$ Not at all to $4=$ Very $m u c h$ so.). The trait cognitive and somatic subscales have excellent internal consistency (cognitive $\alpha=$ .95; somatic $\alpha=.94)$. The state cognitive and somatic subscales also have excellent internal consistency (cognitive $\alpha=.94$; somatic $\alpha=.92$; Ree et al, 2008). In the present study, the internal consistency for the trait cognitive and somatic subscales were $\alpha=.92$ and $\alpha=.91$, respectively. The internal consistency for the state cognitive and somatic subscales were $\alpha=.93$ and $\alpha=.94$, respectively.

The Center for Epidemiological Studies-Depression Scale (CES-D; Radloff, 1977) is a 20-item measure of depressive symptoms experienced in the past 7 days. Examples of items include: "I was bothered by things that don't usually bother me" and "I thought my life has been a failure." Items are rated on a 4 -point scale $(0=$ rarely to $3=$ most or all of the time $)$, total score can range from 0 to 60 with high scores indicating greater depression symptoms. The CES-D is found to have high internal consistency ( $\alpha$ range from .84 to .90; Radloff, 1977). CES-D also has good convergent validity with the Beck Depression Inventory (Eaton, Smith, Ybarra, Muntaner, \& Tien, 2004; Zich, Attkisson, \& Greenfield, 1990). The CES-D demonstrated excellent internal consistency in the present study $(\alpha=.93)$.

\section{Self-report measures of emotional intensity and emotion regulation. The Affect}

Intensity Measure (AIM; Larsen \& Deiner, 1987) is a 40-item self-report measure that assesses people's perception of the frequency at which they experience intense emotions and strong emotional reactions. Sample items include: "When I feel happy, it is a strong type of exuberance," "My heart races at the anticipation of some exciting event." Items are rated on a 6point scale $(1=$ never to $6=$ always $)$. The AIM has excellent internal consistency $(\alpha=.84$; Flett 
$\&$ Hewitt, 1995) and good test-retest reliability at 1 month, 2 months, and 3 months, $.80, .81$ and .81, respectively (Larsen, 1984, cf. Larsen \& Deiner, 1987). Scores on the AIM exhibit strong convergence with people's reported intensity of emotion reactions to negative $(r=.32, p<.01)$ and positive $(r=.43, p<.001)$ events in daily life (Larsen, Diener, \& Emmons, 1986). The internal consistency for the AIM was high in the present study $(\alpha=.91)$.

The Emotional Intensity Scale (EIS; Bachorowski \& Braaten, 1994) is a 30-item selfreport measure that assesses the intensity of trait positive and negative emotions, independent of how frequently the emotion is experienced. Items are rated on a 5-point scale, with lower ratings denoting lesser emotional intensity; each item has a unique scale. A sample item from the negative emotion subscale is "I think about awful things that might happen. I feel." Individuals then choose the correct value from the following scale: $1=$ It has little effect on me, $2=$ A little worried, $3=$ Worried, $4=$ Very worried, $5=$ So extremely worried that $I$ can almost think of nothing else. A sample item from the positive emotion subscale: "When someone compliments me. I feel." The corresponding scale is: $1=$ It has little effect on me, $2=$ Mildly pleased, $3=$ Pleased, $4=$ Very pleased, $5=$ Ecstatic - on top of the world. The EIS has good internal consistency $(\alpha=.90)$ and good test-retest reliability ( $r=.71$ to .87 ; Bachorowski \& Braaten, 1994). The EIS negative and positive subscales demonstrate convergent validity with the AIM (EIS - negative subscale $r=.45, p<.01$; EIS - positive subscale, $r=.48, p<.01$ ). The EISnegative subscale was also associated with elevated scores on the neuroticism subscale of the Eysenck Personality Questionnaire $(r=.64, p<.01)$. The positive subscale was not associated with neuroticism ( $r=.23, p>.05$; Bachorowski \& Braaten, 1994). The EIS-negative subscale and the EIS - positive subscale demonstrated excellent internal consistency, $\alpha=.91$ and $\alpha=.92$ respectively. 
The Difficulties in Emotion Regulation Scale (DERS; Gratz \& Roemer, 2004) is a 36item measure that assesses difficulties with emotion regulation across six dimensions, including lack of emotional awareness (e.g., "I pay attention to how I feel” reverse scored), lack of clarity in emotional experience (e.g., "I have no idea how I am feeling”), nonacceptance of emotions (e.g., "When I am upset, I become angry with myself for feeling that way"), inability to engage in goal-directed behaviour (e.g., When I am upset, I have difficulty getting work done”), difficulty refraining from impulsive behaviour when experiencing negative emotions (e.g., "When I am upset, I become out of control), and the use of ineffective emotion regulation strategies (e.g., "When I am upset, it takes me a long time to feel better"). Each item is rated on a 5-point scale $(1=$ not at all to $5=$ completely $)$ and higher scores are suggestive of greater dysregulation. All subscales of the DERS have shown high internal consistency $(\alpha>.80)$ and good test-retest reliability $(\rho=.88)$. The DERS demonstrates good convergent validity with the Generalized Expectancy for Negative Mood Regulation Scale $(r=-.69, p<.01$; Catanzaro \& Mearns, 1990; Gratz \& Roemer, 2004). The internal consistency for the DERS total score in the present study was excellent $(\alpha=.95)$.

The Emotion Regulation Questionnaire (ERQ; Gross \& John, 2003) is a 10-item selfreport measure of people's perceived trait-like tendencies to engage in expressive suppression (e.g., "I control my emotions by not expressing them") and cognitive reappraisal (e.g., "I control my emotions by changing the way I think about the situation I'm in"). Items are rated on a 7point likert scale $(0=$ strongly disagree to $7=$ strongly agree $)$. The ERQ has good internal consistency (reappraisal subscale: $\alpha=.79$; suppression subscale $\alpha=.73$ ) and good test-retest reliability at 3 months ( $r=.69$ for both subscales; Gross \& John, 2003). The ERQ has also demonstrated good convergent validity with the Negative Mood Regulation Scale (reappraisal 
subscale: $r=.30, p<.05$; suppression subscale: $r=-.22, p<.05$; Catanzaro \& Mearns, 1990; Gross \& John, 2003). In the present study, the cognitive reappraisal subscale and the emotional suppression subscales demonstrated good internal consistency, $\alpha=.88$ and $\alpha=.81$ respectively.

State measures of emotional intensity. Participants' baseline emotional intensity and level of emotional reactivity (i.e., change in emotional intensity from baseline to peak intensity following an emotional stimulus) were assessed via their subjective and physiological responses using the following measures.

Subjective measures. Participants' subjective emotional responses were assessed at baseline (i.e., baseline emotional intensity) and following each emotion induction (i.e., emotional reactivity). Participants were asked to rate the intensity of their current experience of 12 emotions or states on a 9-point scale from 0 (not at all) to 8 (very much). Negative emotion items included: anxious, angry, sluggish, sad, irritable, and distressed. Positive emotion items included: content, relaxed, enthusiastic, joyful, proud, and interested. The emotion items have been used widely in studies that have assessed emotional experiences in "real-time" (e.g., Farmer \& Kashdan, 2013; Nezlek \& Kuppens, 2008). Mean positive and negative emotion scores were calculated by averaging responses to the positive and negative items, respectively. The internal consistencies for the positive emotion ratings and the negative emotion ratings were excellent, $\alpha$ $=.87$ and $\alpha=.86$, respectively.

Physiological measures. Physiological activity was assessed during the baseline task (i.e., baseline emotional intensity) and during each emotion induction task (i.e., emotional reactivity). Skin conductance was monitored as a measure of the sympathetic nervous system, whereas electrocardiogram (ECG) data were recorded to provide a measurement of the parasympathetic nervous system. 
ECG data were collected via disposable electrodes with the MP150 Data Acquisition System and AcqKnowledge 4.2 software (Biopac systems Inc., Santa Barbara, CA) in the Lead II configuration ( 3 electrodes attached: one under the right clavicle, one placed below the lowest left rib and one on the lowest right rib). Data were digitized at 1,000 samples per second. The interbeat intervals (IBI) observed in the ECG data were then used to calculate respiratory sinus arrhythmia (RSA). IBIs were evaluated for artifacts due to movement or software errors and manually edited in CardioEdit (Brain-Body Centre, 2007b). RSA values were then calculated using the standardized procedure developed by Porges (1985) using the CardioBatch software (Brain-Body Centre, 2007a). CardioBatch applies a high band-pass filter to be consistent with spontaneous respiration. The CardioBatch computes an RSA value in the units of $1 \mathrm{~ns}^{2}$. Each of the $5 \mathrm{~m}$ phases (i.e., baseline, vanilla baseline and imagery) were divided into 10 sequential $30 \mathrm{~s}$ epochs of IBI data; therefore, for each $5 \mathrm{~m}$ segment there were $10 \mathrm{RSA}$ values calculated.

Skin conductance was recorded from electrodes attached to the distal phalanges of the participants' nondominant hand. Physiological activity was recorded using a BIOPAC MP150 system. The skin conductance data were cleaned and analyzed using Acqknowledge software. NS-SCR were identified in the data using a threshold of a minimum increase of .02 $\mu$ s and a rejection rate of 10\% (Braithwaite, Watson, Jones, \& Rowe, 2013; Vossel \& Zimmer, 1990).

Emotion induction stimuli. Personalized imagery scripts were developed using the Imagery Script Development Task (adapted from Kuo \& Linehan, 2009; Pitman, Orr, Forgues, de Jong, \& Claiborn, 1987; Yamasaki, 2006). Participants were asked to provide specific details about a positive future event (positive emotion induction) and a worst-case scenario that is related to one of their current worry domains (negative emotion induction). For each scenario, participants were asked to (a) describe the subject of the scenario, (b) elaborate on the visual, 
auditory, tactile, and olfactory components of the scenario, (c) select 5 adjectives that best describe how they would feel in the scenario, and (d) select 5 physical sensations that would best represent their experiences in the scenario. These idiographic details were inserted into a standard imagery script (see Appendix A), which was then audio recorded by the researcher (EJP) in a neutral voice tone and played to participants during the emotion inductions. Similar script-driven imagery procedures have been used to evoke a variety of emotions, as well as disorder-specific mental imagery, including imagery associated with snake phobias and performance anxiety (e.g., Lang, Levin, Miller, \& Kozak, 1983), as well as traumatic events (Pitman et al., 1987).

Post-Induction Manipulation Check. At the end of the study, participants were given a series of questions to answer regarding their degree of engagement, or participation, with the emotion induction tasks. In particular, participants provided ratings on their ability to engage with the task, to stay focused, and to complete the task as instructed, difficulty with following the instructions and if they did anything to distract themselves. Items were rated from 0 to 10 , with a higher score indicating greater engagement with the task or greater use of distraction. Please see Appendix B for the list of questions. Given that poor engagement may attenuate emotional reaction to the stimulus, several researchers have highlighted the importance of asking participants about their level of engagement and/or use of distraction strategies during emotion inductions (e.g., Kuo \& Linehan, 2009).

\section{Procedure}

Each participant attended a single 2-hour laboratory session. The timeline for the laboratory procedure is outlined in Appendix $\mathrm{C}$ and was developed based on published experimental induction procedures (see Kuo \& Linehan, 2009; Pitman et al., 1987; Yamasaki, 
2006). Prior to attending the laboratory session, participants were instructed to avoid ingesting caffeine and tobacco on the day of the session, and to refrain from taking any over the counter medications $24 \mathrm{hrs}$ prior to the session because these substances alter physiological response.

Upon arrival to the laboratory session, the researcher (EJP) provided participants with an overview of the study procedure. Participants then read and signed a consent agreement (see Appendix D). The first study task was completing the Imagery Script Development Task, where participants identified specific details about a hypothetical positive event and a hypothetical negative (i.e., worrisome) event in their life. Participants were then seated in front of a computer and completed the demographics questionnaire, PSWQ, SPIN, CES-D, AIM, EIS, DERS, and ERQ. While participants were completing questionnaires, the experimenter (EJP) audio recorded the imagery scripts in a neutral tone, which were later played back to the participants during the emotion induction tasks.

Upon completion of the questionnaires, participants were informed of the procedures for attaching the physiological monitoring equipment and the sensors were attached. Physiological responses were monitored throughout the remainder of the study. Participants were then seated in front of a computer screen to assess subjective and physiological emotional response. First, participants completed a baseline task to assess baseline subjective and physiological response. Following the baseline task, participants completed the two emotion induction tasks to assess emotional reactivity, which were counterbalanced. All tasks were delivered via EPrime.

Assessment of baseline emotional intensity. To obtain baseline emotional intensity, participants were monitored during a 5-minute baseline task during which they were asked to "maintain wakefulness and sit quietly and still" (Kuo \& Linehan, 2009). Immediately following the "true baseline" task, participants were prompted to provide a rating of their current: (1) 
intensity of positive and negative emotions (i.e., subjective emotion response); (2) level of worry; and (3) level of muscle tension/bodily arousal (see Appendix E).

Assessment of emotional reactivity. Prior to each emotion induction, participants completed a "vanilla baseline," which was a nonstressful cognitive task that required them to count the number of times a specified colour appeared on the computer screen (see Jennings, 1992; Kuo \& Linehan, 2009). The aim of the vanilla baseline is to control for residual emotion that may have been induced in the prior task.

To assess positive and negative emotional reactivity, each participant completed two emotion induction tasks (i.e., one positive and one negative). Following the vanilla baseline, participants listened to the audio recording of their emotional scripts. Participants were asked to listen carefully and attempt to form a "vivid picture" of the scenario and to "focus on the emotions that the scenario brings to mind." Duration of each script was approximately 1 minute. Upon termination of the audio recording, participants were instructed to continue imagining the scenario from beginning to end until they heard a tone (5 minutes). Following the tone, participants were instructed to stop imagining the script and to relax (5 minutes).

Immediately following the task, participants were asked to provide a rating of how vivid their mental imagery was during the imagery task in a scale from 0 (not at all vivid) to 10 (extremely vivid; see Appendix F) (Yamasaki, 2006). Participants then completed a rating of their current: (1) intensity of positive and negative emotions (i.e., subjective emotion response); (2) level of worry; and (3) level of muscle tension/bodily arousal.

The same procedure was used for both positive and negative emotion inductions. The presentation order of the emotion induction tasks (positive and negative) was counterbalanced across participants. Subjective emotional reactivity was operationalized as the change in 
emotional response from prior to the presentation of the emotional script (i.e., following vanilla baseline) to following the 5-minute imagery period.

At the end of the study, participants completed a postmanipulation check during which they were asked to rate their ability to engage with the emotion induction task and the extent to which they tried to attenuate the emotional arousal experienced during the imagery tasks. The electrodes were then removed and participants were debriefed (Appendix G) and compensated (\$25) for their participation.

\section{Statistical Analyses}

Data were analyzed using SPSS statistics, Version 22.0. To examine for outliers, the data were plotted and z-score values greater than an absolute value of 3.29 were identified as outliers (Tabachnick \& Fidell, 2007). Data points that were classified as outliers were replaced by the next extreme value. For all questions that had an a priori hypothesis, planned pairwise comparisons were conducted even when the omnibus $F$ tests were nonsignificant and no adjustments were used (Hancock \& Klockars, 1996). When no a priori hypotheses were stated, the $F$ tests were followed by Tukey posthoc comparisons to explore the main and interaction effects.

Trait self-report questionnaires. A series of univariate analyses of variance (ANOVAs) were conducted to compare the three groups (GAD, SAD, NCC) on the trait self-report questionnaires assessing intensity of emotion and difficulties with emotion regulation.

Subjective responses. A series of univariate ANOVAs were performed to examine differences in baseline emotional intensity (positive and negative) and baseline level of worry and muscle tension/bodily arousal across the three groups (GAD, SAD, NCC). In addition, a series of repeated-measures ANOVAs were performed to compare the three groups on emotional 
reactivity (positive and negative) and change in level of worry and muscle tension/bodily arousal following the positive and negative emotion induction task.

Physiological response. Mixed model ANOVAs were used for examining group differences in RSA and NS-SCRs. The use of mixed model ANOVAs is recommended for the analysis of physiological data given that it accommodates for missing data, accounts for autocorrelation between repeated measurements, and has increased flexibility for modeling time effects (Bagiella, Sloan, \& Heitjan, 2000; Gueorguieva \& Krystal, 2004). Additionally, an advantage of the mixed model ANOVA that it models separate means per group over time. In the present study, there was a specific focus on the group effect (i.e., differences between groups collapsed across epochs) and the interaction effect (Group x Epoch). 


\section{Results}

\section{Preliminary Analyses}

Clinical characteristics. A series of univariate ANOVAS were performed to compare participants' scores on the PSWQ, SPIN, State and Trait STICSA, and CES-D and to confirm differences in clinical characteristics across the three groups (GAD, SAD, NCC; see Table 2). On the PSWQ, a measure of self-reported worry levels, there was a significant between-groups effect. The GAD group scored significantly higher on the PSWQ than the SAD group and the NCC group. The SAD group also scored higher on the PSWQ compared to the NCC group. In addition, on scores from the SPIN, a measure of social anxiety symptoms, there was a significant between-groups effect. The GAD group and NCC group scored significantly lower on the SPIN compared to the SAD group. The findings provide support that the three groups differ on their self-reported symptoms of worry and social anxiety, and specifically, that the GAD group reported elevated worry relative to the comparison groups, whereas the SAD group reported elevated symptoms of social anxiety relative to the GAD group and the NCC group.

On the STICSA-State, a measure of current somatic and cognitive symptoms of anxiety, significant differences were found across the groups. The GAD group reported significantly greater cognitive symptoms of anxiety compared to the SAD group and the NCC group. On the somatic symptoms subscale, the GAD group reported elevated scores compared to NCC group, but did not differ from the SAD group. On the STICSA-Trait, a measure of general somatic and cognitive symptoms of anxiety, the GAD group reported greater symptoms of both somatic and cognitive anxiety compared the NCC group, but did not differ from the SAD group. Finally, on the CES-D, a measure of current depression symptoms, the GAD group reported significantly greater severity of depression symptoms relative to that of the NCC group, but did not differ 
significantly from the SAD group. The SAD group also reported greater severity of depression symptoms compared to that of the NCC group. Taken together, the findings on the self-report questionnaires of anxiety and mood related symptoms provide additional support of the differences in clinical characteristics across the three groups of participants. 
Table 2

Clinical Characteristics of the GAD, SAD and NCC groups

\begin{tabular}{|c|c|c|c|c|c|c|c|c|c|c|c|c|c|}
\hline & \multicolumn{2}{|c|}{$\begin{array}{c}\mathrm{GAD} \\
n=22\end{array}$} & \multicolumn{2}{|c|}{$\begin{array}{c}\text { SAD } \\
n=23\end{array}$} & \multicolumn{2}{|c|}{$\begin{array}{c}\mathrm{NCC} \\
n=20\end{array}$} & \multirow{2}{*}{$\begin{array}{c}\text { ANOVA } \\
\text { F (df) }\end{array}$} & \multicolumn{2}{|c|}{ GAD vs. SAD } & \multicolumn{2}{|c|}{ GAD vs. NCC } & \multicolumn{2}{|c|}{ SAD vs. NCC } \\
\hline & Mean & $S D$ & Mean & $S D$ & Mean & $S D$ & & $p$ & $d$ & $p$ & $d$ & $p$ & $d$ \\
\hline PSWQ & 67.14 & 6.50 & 57.96 & 11.58 & 31.70 & 9.18 & $100.95^{\mathrm{a}}(2,38.56)^{* * *}$ & $<.01$ & 0.98 & $<.001$ & 4.46 & $<.001$ & 2.51 \\
\hline SPIN & 29.45 & 16.76 & 40.91 & 10.57 & 4.85 & 4.89 & $121.10^{\mathrm{a}}(2,34.84)^{* * *}$ & $<.01$ & 0.82 & $<.001$ & 1.99 & $<.001$ & 4.38 \\
\hline \multicolumn{14}{|l|}{ STICSA } \\
\hline $\begin{array}{l}\text { State } \\
\text { Cognitive }\end{array}$ & 25.45 & 6.39 & 18.83 & 7.60 & 11.20 & 1.94 & $56.03^{\mathrm{a}}(2,32.99)^{* * *}$ & $<.01$ & 0.94 & $<.001$ & 3.02 & $<.001$ & 1.38 \\
\hline $\begin{array}{l}\text { State } \\
\text { Somatic }\end{array}$ & 21.50 & 8.29 & 16.91 & 6.17 & 11.50 & 0.76 & $23.74^{\mathrm{a}}(2,29.40)^{* * *}$ & .10 & 0.63 & $<.001$ & 1.70 & .001 & 1.23 \\
\hline $\begin{array}{l}\text { Trait } \\
\text { Cognitive }\end{array}$ & 26.41 & 6.19 & 24.96 & 6.34 & 13.45 & 2.63 & $60.76(2,36.64)^{* * *}$ & .72 & 0.23 & $<.001$ & 2.73 & $<.001$ & 2.37 \\
\hline $\begin{array}{l}\text { Trait } \\
\text { Somatic }\end{array}$ & 23.05 & 6.05 & 22.96 & 5.45 & 14.10 & 2.57 & $36.74^{\mathrm{a}}(2,37.30)^{* * *}$ & .99 & 0.02 & $<.001$ & 1.93 & $<.001$ & 2.08 \\
\hline CES-D & 23.18 & 12.29 & 19.39 & 9.91 & 5.10 & 5.39 & $30.46^{\mathrm{a}}(2,38.16)^{* * * *}$ & .50 & 0.34 & $<.001$ & 1.91 & $<.001$ & 1.79 \\
\hline
\end{tabular}

Note. GAD = generalized anxiety disorder; $\mathrm{SAD}=$ social anxiety disorder; $\mathrm{NCC}=$ nonclinical control; $\mathrm{PSWQ}=$ Penn State Worry Questionnaire; SPIN = Social Phobia Inventory; STICSA = State-Trait Inventory of Cognitive and Somatic Anxiety; CES-D = Center for Epidemiological Studies - Depression. Bold indicates a significant difference $* * * p<.001$

${ }^{\mathrm{a}}=$ Welch F and Games-Howell Planned Comparisons 


\section{Manipulation Checks}

Intensity of the emotion induction scripts. To determine if the idiographic scripts for the negative and positive emotion induction tasks had equivalent levels of emotional intensity across three groups (GAD, SAD, NCC), two independent raters, who were blind to group identification, rated the scripts using Strauss and Allen's (2008) emotional intensity coding scheme. The interrater reliability was high for the ratings of the negative emotion induction scripts $($ ICC $=.78)$ and the positive emotion induction scripts $(\mathrm{ICC}=.78)$. Separate univariate ANOVAs were performed on the average emotional intensity ratings provided for the negative emotion induction (GAD: $M=6.13, S D=0.60 ; \mathrm{SAD}: M=6.00, S D=0.58$; NCC: $M=5.85, S D=0.81$ ) and for the positive emotion induction (GAD: $M=5.32, S D=0.68$; SAD: $M=5.67, S D=0.75$; NCC: $M=$ $5.55, S D=0.71)$ to determine if the three groups had equivalent scripts. There was no betweengroups effect for the negative emotion induction, $F(2,62)=1.48, p=.24$, or the positive emotion induction, $F(2,62)=0.96, p=.39$. The findings indicate that the three groups produced scripts with equivalent intensity ratings and as a result, emotional intensity rating was not included as a covariate in the subsequent analyses examining reactivity to the emotion induction tasks.

Postinduction check of participant engagement. At the end of the study, participants answered a series of questions regarding their level of participation during emotion induction tasks. A series of univariate ANOVAs were performed to determine if participants' level of engagement with the two emotion induction tasks differed across the three groups (GAD, SAD, NCC; see Table 3). For the negative emotion induction task, there were no differences found across the three groups in their reported ability to engage in the task, to stay focused on the task, to follow the task instructions, or in their reported use of distraction during the task (all $p \mathrm{~s}>.05$ ). It is noted that participants endorsed some degree of distraction during the negative emotion 
induction; when asked to describe what they did as a distraction, a few participants commented that they were, "thinking reassuring thoughts," "not thinking too deeply about the stressful scenario," and "thinking of ways to prevent the scenario from happening."

For the positive emotion induction task, there were also no differences among the three groups in their reported ability to focus on the task, to follow the task instructions, difficulty with following the instructions, or in their reported use of distraction during the task (all $p s>.05$ ). Regarding engagement with the positive emotion induction task, however, there was a significant between-group difference. Posthoc comparisons revealed that the SAD group reported lower engagement with the task compared to the NCC group $(p=.03, d=0.82)$, but did not differ from the GAD group $(p=.72 . d=0.59)$. There was no difference in reported level of engagement between the GAD and NCC groups. The findings suggest that the SAD group reported lower engagement in the mental imagery task relative to the NCC group. Although the GAD group reported lower engagement compared to the NCC group and greater engagement compared to the SAD group, the differences were not statistically significant. 
Table 3

Means and Standard Deviations of Emotion Induction Engagement Questions by Group

\begin{tabular}{|c|c|c|c|c|c|c|c|c|c|}
\hline & \multicolumn{2}{|c|}{$\begin{array}{l}\text { GAD } \\
n=22\end{array}$} & \multicolumn{2}{|c|}{$\begin{array}{c}\text { SAD } \\
n=21^{\mathrm{a}}\end{array}$} & \multicolumn{2}{|c|}{$\begin{array}{l}\text { NCC } \\
n=20\end{array}$} & \multirow[b]{2}{*}{$F(\mathrm{df})$} & \multirow[b]{2}{*}{$p$} & \multirow[b]{2}{*}{$\eta_{p}^{2}$} \\
\hline & M & SD & M & SD & M & SD & & & \\
\hline \multicolumn{10}{|c|}{ Negative Emotion Induction } \\
\hline Engage & 6.80 & 2.12 & 6.19 & 2.34 & 7.05 & 2.01 & $0.86(2,60)$ & .43 & .03 \\
\hline Focus & 6.36 & 2.13 & 5.90 & 2.49 & 6.55 & 2.28 & $0.43(2,60)$ & .65 & .01 \\
\hline Complete task & 7.55 & 2.18 & 7.05 & 2.58 & 8.10 & 1.86 & $1.11(2,59)$ & .34 & .04 \\
\hline $\begin{array}{l}\text { Follow } \\
\text { instructions }\end{array}$ & 7.55 & 2.63 & 6.19 & 3.20 & 7.50 & 2.26 & $1.67(2,60)$ & .20 & .05 \\
\hline Distraction & 2.59 & 2.17 & 3.86 & 2.83 & 2.60 & 1.88 & $2.05(2,60)$ & .14 & .06 \\
\hline
\end{tabular}

Positive Emotion Induction

$\begin{array}{lccccccccc}\text { Engage } & 7.59 & 1.82 & 6.43 & 2.13 & 8.05 & 1.82 & 3.88(2,60) & \mathbf{. 0 3} & .12 \\ \text { Focus } & 7.00 & 2.20 & 6.05 & 2.11 & 7.30 & 2.03 & 1.98(2,60) & .15 & .06 \\ \text { Complete task } & 7.82 & 1.99 & 7.62 & 2.36 & 8.60 & 1.61 & 1.07(2,60) & .35 & .04 \\ \begin{array}{l}\text { Follow } \\ \text { instructions }\end{array} & 8.00 & 2.31 & 7.05 & 2.67 & 8.60 & 1.43 & 2.58(2,60) & .08 & .08 \\ \begin{array}{l}\text { Distraction } \\ \text { C }\end{array} & 1.73 & 0.98 & 2.71 & 2.26 & 1.75 & 1.59 & 2.35(2,60) & .10 & .07\end{array}$

Note. $\mathrm{GAD}=$ generalized anxiety disorder $\mathrm{SAD}=$ social anxiety disorder $; \mathrm{NCC}=$ nonclincal control. Items were rated on a scale from 0 to 10 , with $10=$ greater engagement or greater use of distraction. Bold indicates a significant between-group difference.

a Two participants did not complete the end of study questionnaire. 


\section{Group Differences on Trait Questionnaires of Emotion and Emotion (Dys)regulation}

To examine between-group differences on measures of trait emotional intensity, univariate ANOVAs were performed to compare participants' scores on the AIM and on the negative and positive emotional intensity subscales of the EIS (see Table 4). Significant between-group differences were found on the AIM. Pairwise comparisons showed the GAD group reported greater frequency of intense emotions compared to the NCC group $(d=0.94)$, but there were no differences between the GAD group and the SAD group $(d=0.34)$ on reported frequency of intense emotions. On the negative affect subscale of the EIS, there were significant betweengroup differences; the GAD group reported greater intensity of negative emotions compared to the SAD group $(d=2.14)$ and the NCC group $(d=3.20)$. On the positive emotional intensity subscale of the EIS, however, the three groups reported no significant differences in intensity of positive emotions.

Next, a univariate ANOVA was conducted to examine differences on self-reported difficulty with emotion regulation among the three groups (GAD, SAD, NCC; see Table 5). There was a significant between-groups effect. Posthoc pairwise comparisons revealed that the GAD group had significantly greater scores on the DERS compared to the NCC group $(d=2.14)$, but did not differ significantly from the SAD group $(d=0.28)$. A secondary examination of differences in scores on the DERS subscales between the GAD group and the NCC group revealed that the GAD group reported greater difficulty with nonacceptance of emotions, engaging in goal-directed behaviour, impulse control, emotional clarity and use of effective emotion regulation strategies compared the NCC group, but the two groups did not differ on their reported emotional awareness $(d=0.36)$. Finally, on the ERQ, there was a significant betweengroups effect. Posthoc pairwise comparisons showed that the GAD group reported significantly 
lower use of cognitive reappraisal compared to the NCC group $(d=1.04)$, but did not differ from the SAD group $(d=0.45)$. There were no between-group differences on reported use of emotional suppression. 
Table 4

Means and Standard Deviations on Self-Report Measures of Trait Emotional Intensity

\begin{tabular}{|c|c|c|c|c|c|c|c|c|c|c|c|c|c|}
\hline & \multicolumn{2}{|c|}{$\begin{array}{c}\text { GAD } \\
n=22\end{array}$} & \multicolumn{2}{|c|}{$\begin{array}{c}\text { SAD } \\
n=23\end{array}$} & \multicolumn{2}{|c|}{$\begin{array}{c}\mathrm{NCC} \\
n=20\end{array}$} & \multirow{2}{*}{$\begin{array}{c}\text { ANOVA } \\
F(\mathrm{df})\end{array}$} & \multicolumn{2}{|c|}{$\begin{array}{c}\text { GAD vs. } \\
\text { SAD }\end{array}$} & \multicolumn{2}{|c|}{$\begin{array}{c}\text { GAD vs. } \\
\text { NCC }\end{array}$} & \multicolumn{2}{|c|}{$\begin{array}{l}\text { SAD vs. } \\
\text { NCC }\end{array}$} \\
\hline & Mean & SD & Mean & SD & Mean & $\mathrm{SD}$ & & $p$ & $d$ & $p$ & $d$ & $p$ & $d$ \\
\hline AIM & 3.86 & 0.62 & 3.66 & 0.57 & 3.34 & 0.48 & $4.58(2,62)^{*}$ & .23 & 0.34 & .01 & 0.94 & .07 & 0.61 \\
\hline \multicolumn{14}{|l|}{ EIS } \\
\hline Positive & 50.50 & 10.33 & 46.26 & 8.59 & 49.25 & 6.61 & $1.41(2,62)$ & .11 & 0.45 & .65 & 0.14 & .27 & 0.39 \\
\hline Negative & 62.82 & 4.96 & 58.61 & 7.46 & 42.70 & 7.39 & $52.07^{\mathrm{a}}(2,39.09)^{* * *}$ & .04 & 0.66 & $<.001$ & 3.20 & $<.001$ & 2.14 \\
\hline
\end{tabular}

Note. $\mathrm{GAD}=$ generalized anxiety disorder; $\mathrm{SAD}=$ social anxiety disorder $; \mathrm{NCC}=$ nonclinical control; $\mathrm{AIM}=$ Affect Intensity

Measure; EIS = Emotion Intensity Scale. Bold indicates a significant difference.

${ }^{* * *} p<.001 ; * p<.05$ 
Table 5

Means and Standard Deviations on Self-Report Measures of Emotion Regulation

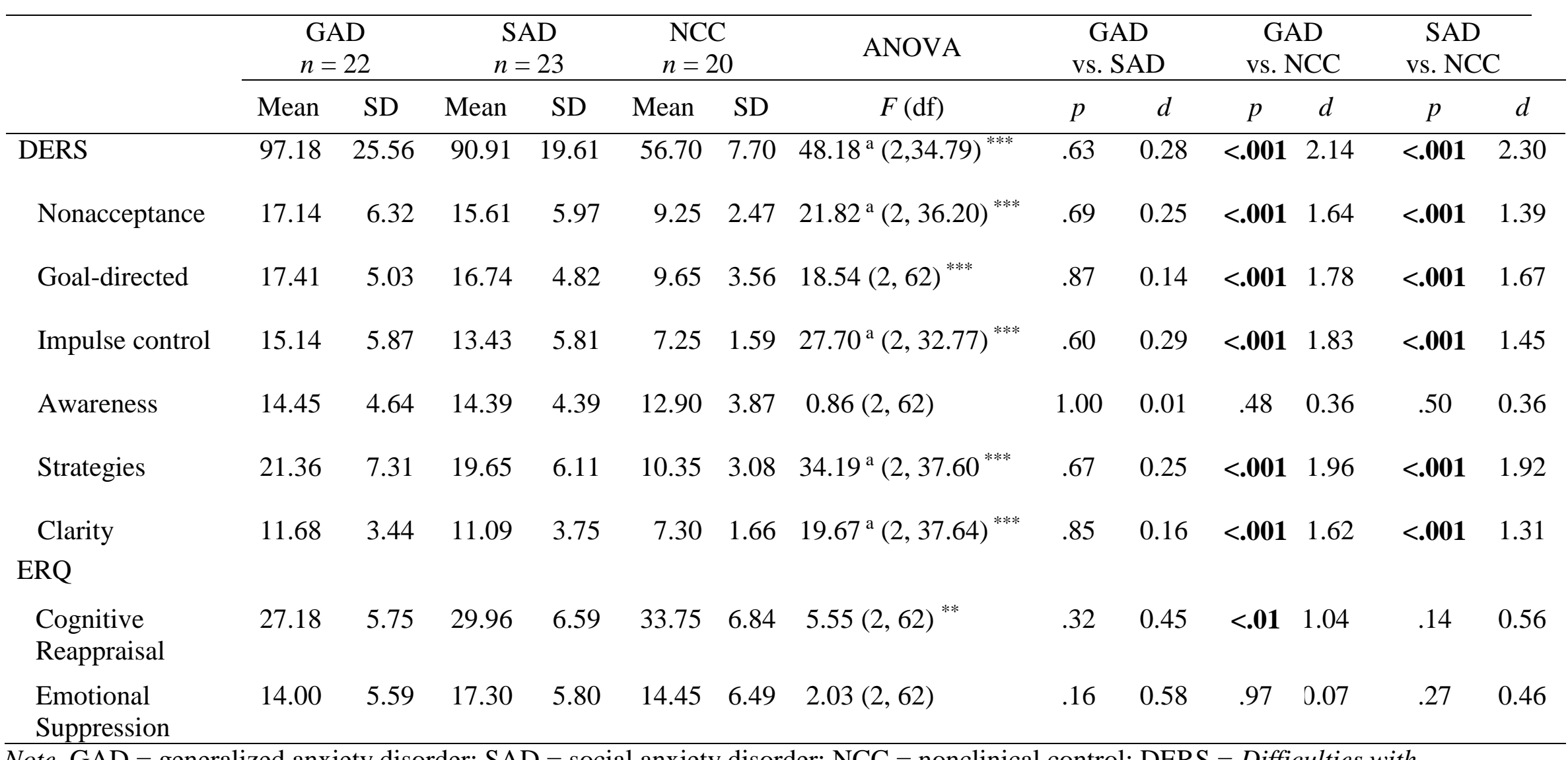

Note. $\mathrm{GAD}=$ generalized anxiety disorder; $\mathrm{SAD}=$ social anxiety disorder; $\mathrm{NCC}=$ nonclinical control; DERS $=$ Difficulties with

Emotion Regulation Scale; ERQ= Emotion Regulation Questionnaire. Bold indicates a significant difference.

${ }^{* * *} p<.001 ;{ }^{* *} p<.01$ 


\section{Objective 1: Examination of Baseline Emotional Intensity}

Baseline emotional intensity was assessed via subjective emotional response and physiological response. Table 6 displays the means and standard deviations for the measures of baseline emotional intensity.

Baseline subjective emotional intensity. Regarding baseline subjective emotional intensity, it was hypothesized that people with GAD would report greater intensity of negative emotions at baseline (i.e., in the absence of provocation) compared to people with SAD and people with no psychopathology. Related to the experience of positive emotions, it was predicted that people with GAD would report significantly lower intensity of positive emotions at baseline compared to the NCC group, but greater intensity compared to the SAD group.

A univariate ANOVA was performed on subjective intensity of negative emotions with group (GAD, SAD, NCC) as the between-groups factor. There was a significant between-groups effect, $F(2,62)=13.16, p<.001, \eta_{p}^{2}=.30$. A priori pairwise comparisons revealed that the GAD group reported greater subjective intensity of negative emotions compared to the SAD group, $t(43)=2.10, p=.04, d=0.63$, and the NCC group, $t(40)=5.1, p<.001, d=1.61$. The SAD group also reported greater subjective intensity of negative emotions relative to the NCC group, $t(41)=3.53, p<.001, d=1.09$.

Next, a univariate ANOVA was performed on intensity of positive emotions with group (GAD, SAD, NCC) as the between-groups factor. There was a significant between-groups effect, $F(2,62)=8.65, p<.001, \eta_{p}^{2}=.22$. A priori pairwise comparisons found that the GAD group reported significantly lower positive emotional intensity compared to the NCC group, $t(40)=$ $2.61, p=.01, d=0.80$, but did not differ significantly from the SAD group, $t(43)=1.60, p=$ $.12, d=0.48$. The $\mathrm{SAD}$ group also reported significantly lower intensity of positive emotions 
compared to the NCC group, $t(41)=3.91, p=.001, d=1.19$. Taken together, at baseline, people with GAD reported greater subjective intensity of negative emotions relative to people with SAD and people with no psychopathology. In addition, people with GAD reported lower intensity of positive emotions relative to that of people with no psychopathology, but equal intensity of positive emotions relative to the emotions reported by people with SAD. 
Table 6

Baseline Emotional Intensity as Assessed via Subjective Emotion Rating, RSA, and SCR

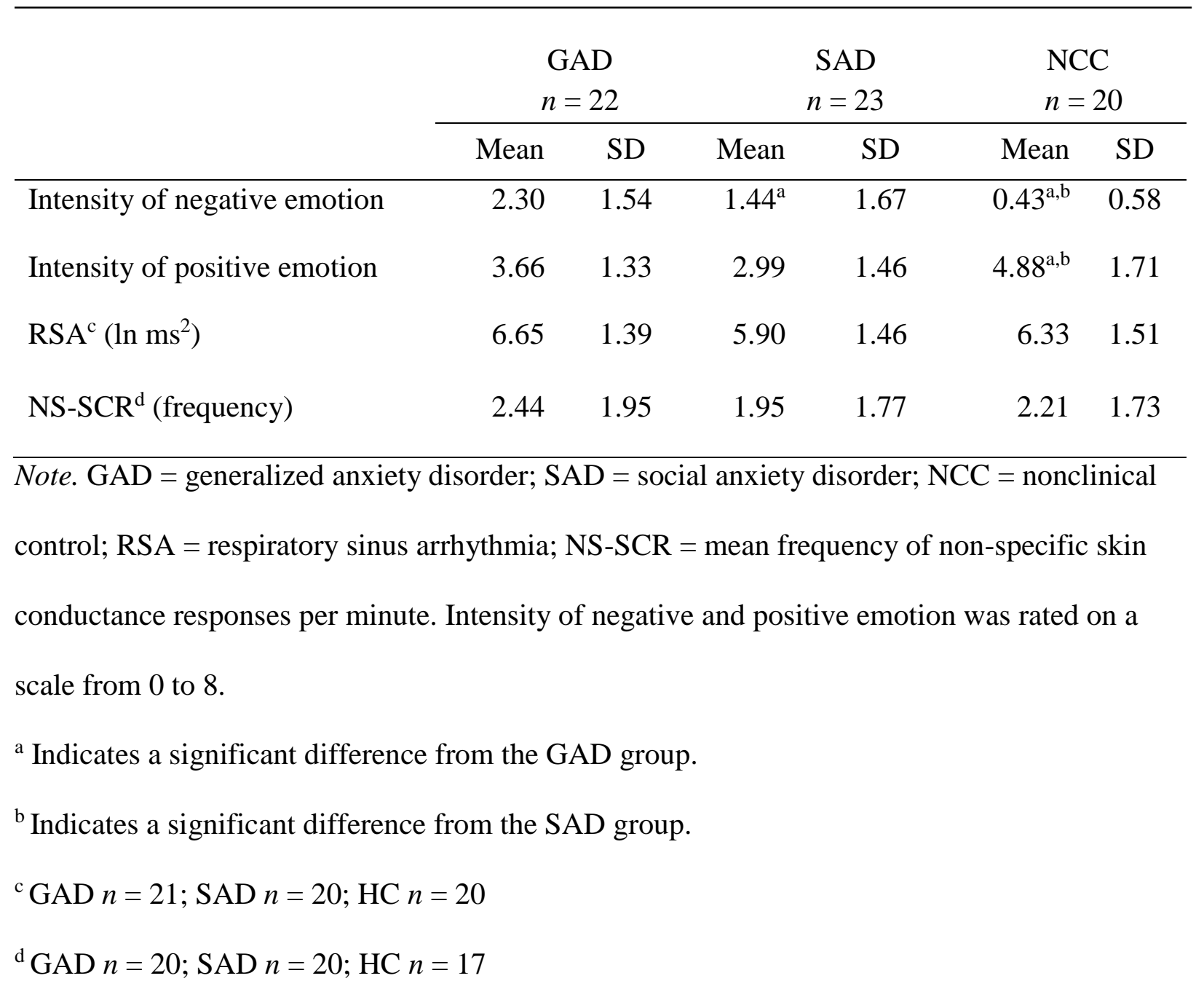


Baseline physiological response. Regarding baseline physiology, it was hypothesized that people with GAD would display reduced RSA compared to people with SAD and people with no psychopathology. A mixed model ANOVA was performed on RSA with Group (GAD, $\mathrm{SAD}, \mathrm{NCC})$ as the between-subjects factor and Epoch $\left(9\right.$ epochs $^{1}, 30 \mathrm{~s}$ each $)$ as the withinsubjects factor. There was no main effect of Group, $F(2,58)=1.55, p=.22$ and no Group $\mathrm{x}$ Time interaction, $F(16,58)=0.70, p=.78$. Planned pairwise comparisons of the betweensubjects effect showed no significant differences in RSA between any of the groups (all $p \mathrm{~s}>$ .05 ), which indicates that the GAD group did not significantly differ from the two control groups on baseline vagal tone.

To test the hypothesis that there would be no significant difference between the groups on their frequency of NS-SCR at baseline, a mixed model ANOVA was performed with Group (GAD, SAD, NCC) as between-subjects factor and Epoch (5 epochs, $60 \mathrm{~s}$ each) as the withinsubjects factor. There was no main effect of Group, $F(2,54.07)=0.72, p=.49$ and no Group $\mathrm{x}$ Epoch interaction, $F(8,214.26)=1.58, p=.13$. Planned pairwise comparisons of the betweensubjects effect indicated that the GAD group did not differ in frequency of NS-SCRs compared to the SAD group ( $p=.56, d=0.26)$ or NCC group $(p=.94, d=0.12)$. Thus, the GAD group did not differ from the two control groups in their baseline frequency of NS-SCRs.

Baseline level of worry and level of muscle tension/bodily arousal. A univariate ANOVA was conducted to examine differences in self-reported baseline level of worry across the three groups (GAD, SAD, NCC). There was a significant between-groups effect, $F(2,62)=$ $13.82, p<.001, \eta_{p}^{2}=.31$. Posthoc comparisons indicated that the GAD group $(M=3.27, S D=$ 2.29) reported greater levels of worry at baseline compared to the NCC group $(M=0.40, S D=$

\footnotetext{
${ }^{1}$ Each $5 \mathrm{~m}$ phase (baseline, vanilla baseline, imagery) was truncated to $4.5 \mathrm{~m}$ to account for missing RSA data in the last $30 \mathrm{~s}$ of the task.
} 
$0.75 ; p<.001, d=1.68)$, but did not differ from the SAD group $(M=2.48, S D=1.95 ; p=.31, d$ $=0.37)$. The $\mathrm{SAD}$ group also reported greater worry compared to the NCC group $(p=.001, d=$ $1.41)$.

Secondary analyses showed that greater baseline worry was associated with greater subjective intensity of negative emotions $(r=.73, p<.001)$, but was not associated with the intensity of baseline positive emotions $(r=-.29, p=.10)$. Regarding physiological response, there was no association between baseline worry and resting RSA $(r=.13, p=.31)$ or frequency of NS-SCRs $(r=-.02, p=.87)$. To account for the significant association between level of worry and intensity of negative emotions, adjusted analyses were performed to examine differences in baseline intensity of negative emotions across the three groups (GAD, SAD, NCC) with level of worry entered as a covariate. With level of worry entered as a covariate, the between-groups effect was no longer significant, $F(2,61)=2.66, p=.08, \eta_{p}^{2}=.08$, but the level of worry covariate was significant, $F(1,61)=36.59, p<.001, \eta_{p}^{2}=.38$. Follow-up pairwise comparisons showed that after controlling for the variance accounted for by level of worry, there was no longer a significant difference between the GAD group $(M$ adjusted $=1.83, S E=.22)$ and SAD group ( $M$ adjusted $=1.30, S E=.20 ; p=.06)$ on baseline negative emotional intensity. However, the difference between the GAD group and NCC group ( $M$ adjusted $=1.10, S E=.24$; $p=.04)$ remained significant. Therefore, level of worry at baseline may account for the significant difference between the GAD group and the SAD group on their reported negative emotional intensity.

Next, a univariate ANOVA was conducted to investigate differences in reported muscle tension/bodily arousal at baseline across the three groups (GAD, SAD, NCC). There was a significant between-groups effect, $F(2,62)=8.25, p=.001, \eta_{p}^{2}=.21$. Posthoc comparisons 
revealed that the GAD group $(M=3.45, S D=2.20)$ reported significantly greater muscle tension/ bodily arousal compared to the NCC group $(M=1.05, S D=1.61 ; p=.19, d=1.25)$, but did not differ from the SAD group $(M=2.37, S D=1.88 ; p=.19, d=0.52)$. The SAD group also reported greater muscle tension and bodily arousal compared to the NCC group $(p=.19, d=$ $0.77)$.

Greater muscle tension/ bodily arousal was associated with greater intensity of negative emotions $(r=.57, p<.001)$, but was not associated with intensity of positive emotions $(r=-.04$, $p=.78)$. There was no association found between baseline self-reported muscle tension/bodily arousal and resting RSA $(r=-.15, p=.24)$ or frequency of NS-SCRs $(r=.06, p=.67)$. To account for the significant association between level of muscle tension/bodily arousal and intensity of negative emotions, differences in baseline intensity of negative emotions across the three groups (GAD, SAD, NCC) was reanalysed including level of muscle tension/bodily arousal as a covariate. The between-groups effect remained significant, $F(2,61)=5.45, p=.01, \eta_{p}^{2}=$ .15. The muscle tension/ bodily arousal covariate was also significant, $F(1,61)=13.92, p<$ $.001, \eta_{p}^{2}=.19$. Posthoc comparisons indicated that after controlling for the variance accounted for by level of muscle tension/bodily arousal, there was no longer a significant difference between the GAD group $(M$ adjusted $=1.99, S E=.24)$ and $\operatorname{SAD}$ group $(M$ adjusted $=1.43, S E=$ $.22 ; p=.09)$ on reported subjective intensity of negative emotions. However, the difference between the GAD group and NCC group ( $M$ adjusted $=0.77, S E=.26 ; p=.01)$ remained significant. Therefore, level of muscle tension/bodily arousal also appears to account for a proportion of the variance in the group difference between people with GAD and those with SAD in their reported intensity of negative emotions. 


\section{Objective 2: Between Group Differences in Emotional Reactivity to Mental Imagery}

Objective 2a: Reactivity to the negative emotion induction. Emotional reactivity was assessed via change in subjective emotional response and change in physiological response. Table 7 displays the means and standard deviations for the measures of emotional reactivity to the negative emotion induction.

Vividness of mental imagery. Following the emotion induction task, participants rated the level of vividness of their mental imagery was on a scale from 0 (not at all vivid) to 9 (extremely vivid). Vividness mean scores separated by group were as follows: GAD: $M=6.38$, $S D=1.83$; SAD: $M=6.00, S D=1.68$; NCC: $M=6.30, S D=1.84$. A univariate ANOVA was performed to examine differences in self-reported vividness of mental imagery during the negative emotion induction across the three groups (GAD, SAD, NCC). There was no betweengroups effect, $F(2,61)=.34, p=.72, \eta_{p}^{2}=.01$, which suggests that the three groups reported equivalent levels of vividness during the negative mental imagery task. Therefore, vividness of mental imagery was not included as a covariate in subsequent analyses. 
Table 7

Emotional Reactivity to the Negative Emotion Induction

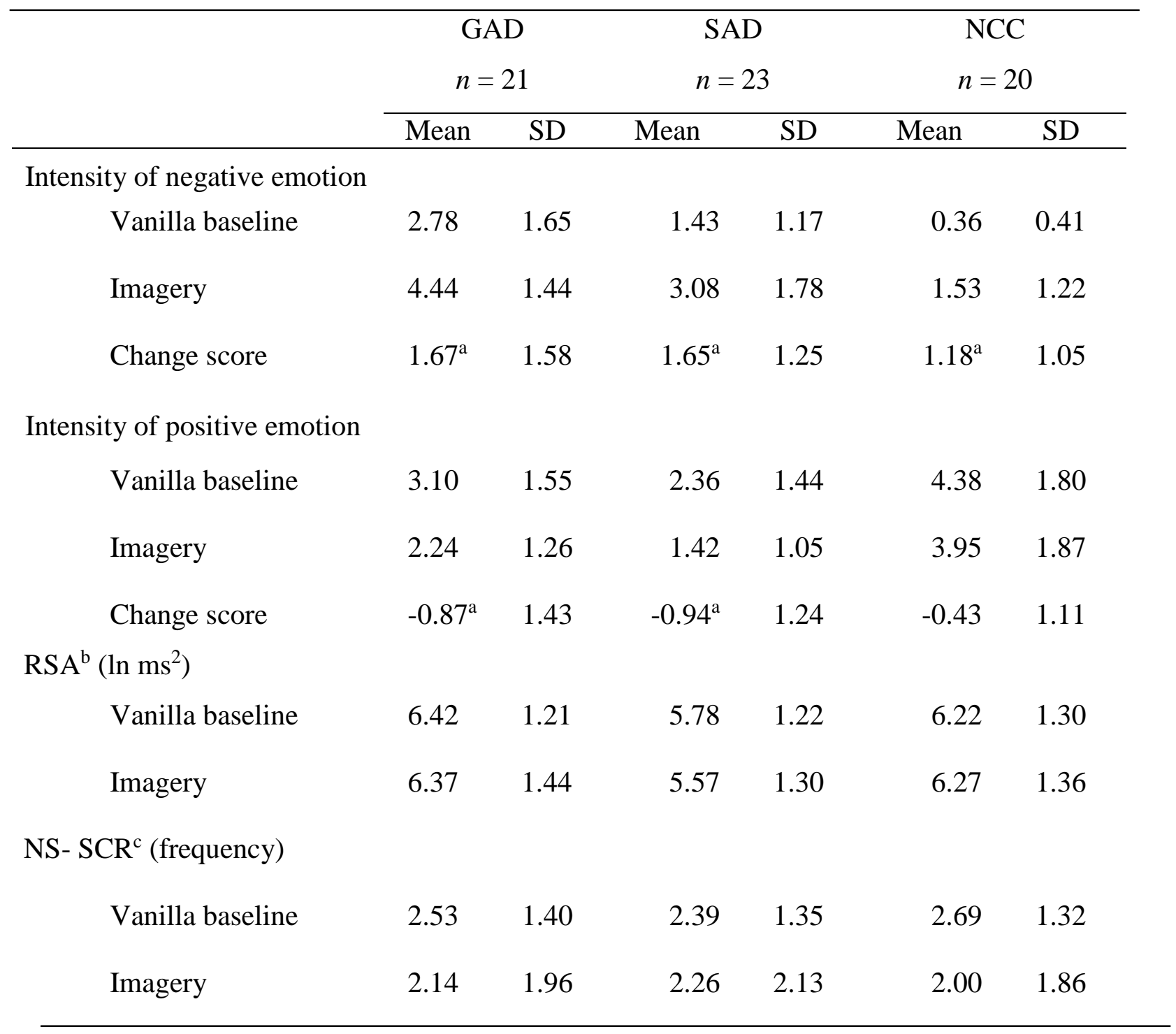

Note GAD = generalized anxiety disorder; $\mathrm{SAD}=$ social anxiety disorder NCC $=$ nonclinical control; RSA = respiratory sinus arrhythmia; NS-SCR = mean frequency of non-specific skin conductance responses per minute.

${ }^{a}$ Indicates a significant within-person effect from baseline to imagery.

${ }^{\mathrm{b}} \operatorname{GAD} n=21 ; \operatorname{SAD} n=21, \operatorname{HC} n=20$

${ }^{\mathrm{c}} \operatorname{GAD} n=19 ; \operatorname{SAD} n=18, \mathrm{HC} n=16$ 
Subjective emotional reactivity. Regarding changes in intensity of negative emotion following a negative emotion induction, it was hypothesized that people with GAD would report a greater increase in the intensity of negative emotion compared to people with SAD and people with no psychopathology. A repeated-measures ANOVA was performed on negative emotional intensity ratings with Time (Vanilla baseline, Imagery) as the within-subjects factor and Group $(\mathrm{GAD}, \mathrm{SAD}, \mathrm{NCC})$ as the between-subjects factor. There was a main effect of Time, $F(1,61)=$ $72.08, p<.001, \eta_{p}^{2}=.54$, and a main effect of Group, $F(2,61)=26.69, p<.001, \eta_{p}^{2}=.47$, but no Time $\mathrm{x}$ Group interaction, $F(2,61)=0.81, p=.45, \eta_{p}^{2}=.03$.

Planned pairwise comparisons of the within-subjects effect revealed that participants reported a significant increase in negative emotional intensity from baseline to following the negative emotion induction (all $p s<.05$ ), which indicates that the emotion induction was effective in inducing a negative emotional response. Next, a priori pairwise comparisons were conducted to determine between-group differences on negative emotional reactivity scores, which were calculated as the difference between the emotion rating during the baseline prior to the emotion induction and the emotion rating immediately following the emotion induction. Contrary to the hypothesis, the GAD group did not exhibit greater subjective negative emotional reactivity compared to the reactivity scores for the SAD group, $t(42)=.25, p=.80, d=0.08$, or the NCC group, $t(38.94)=1.81, p=.25, d=0.37$.

Regarding reactivity of positive emotions, it was hypothesized that participants would report a decrease in the intensity of positive emotions following the negative emotion induction. A repeated-measures ANOVA was performed on subjective emotional intensity of positive emotion with Time (Vanilla baseline, Imagery) as the within-subjects factor and Group (GAD, $\mathrm{SAD}, \mathrm{NCC})$ as the between-subjects factor. There was a main effect of Time, $F(1,61)=18.61$, 
$p=<.001, \eta_{p}^{2}=.23$, and a main effect of Group $F(2,61)=15.23, p=<.001, \eta_{p}^{2}=.33$, but no Time x Group interaction, $F(2,61)=0.84, p=.44, \eta_{p}^{2}=.03$.

Planned pairwise comparisons of the within-subjects effect revealed that the GAD group $(p<.01)$ and SAD group $(p<.01)$ reported a significant decrease in intensity of positive emotions from baseline to following the negative emotion induction, but the NCC group did not report a significant change $(p=.17)$. Next, a priori pairwise comparisons were performed to determine between-subject differences in positive emotional reactivity scores, which were calculated as the difference between the emotion rating during the baseline prior to the emotion induction and the emotion rating immediately following the emotion induction. The GAD group did not exhibit significant differences in their positive emotion change scores compared to the SAD group, $t(42)=0.10, p=.92, d=0.03$, or the NCC group, $t(39)=1.10, p=.28, d=0.34$.

Physiological reactivity. Based on past findings that people with GAD exhibit physiological inflexibility, it was predicted that the GAD group would exhibit a lesser degree of reactivity in RSA compared to the control groups. A mixed model ANOVA on RSA was performed with Group (GAD, SAD, NCC) as the between-subjects factor and Epoch (9 epochs, $30 \mathrm{~s}$ each) as the within-subjects factor. Mean RSA from the vanilla baseline prior to the negative emotion induction task was entered as a covariate. There was no main effect of Group, $F(2,56)=0.14, p=.87$, and no Group x Epoch interaction, $F(16,448)=0.98, p=.48$. Planned pairwise comparisons of the between-subjects effect indicated that the GAD group did not differ from the SAD group $(p=.68)$ or the NCC group $(p=.99)$ in degree of RSA reactivity.

In terms of sympathetic reactivity, it was predicted that the GAD group would report a lower frequency of NS-SCRs during the negative emotion induction compared to the SAD group and NCC group. A mixed model ANOVA was performed on frequency of NS-SCRs during the 
emotion induction task with Group (GAD, SAD, NCC) as the between-subjects factor and Epoch (5 epochs, $60 \mathrm{~s}$ each) as the within-subjects factor. Frequency of NS-SCRs during the vanilla baseline was entered as a covariate. There was no main effect of Group, $F(2,47)=0.09, p=.92$, and no Group x Epoch interaction, $F(8,188)=0.68, p=.71$. Planned pairwise comparisons of the between-groups effect found no difference between the GAD group and the SAD group $(p=$ $.50)$ or the NCC group $(p=.50)$ in frequency of NS-SCRs during the negative emotion induction task.

Worry and muscle tension/bodily arousal reactivity. To examine changes in level of worry following the negative emotion induction, a repeated-measures ANOVA on level of worry was performed with Time (Vanilla baseline, Imagery) as the within-subjects factor and Group $(\mathrm{GAD}, \mathrm{SAD}, \mathrm{NCC})$ as the between-subjects factor. There was a main effect of Time, $F(1,61)=$ $59.70, p<.001, \eta_{p}^{2}=.50$, a main effect of Group, $F(2,61)=17.65, p<.001, \eta_{p}^{2}=.37$, but no Time $\mathrm{x}$ Group interaction, $F(2,61)=0.52, p<.60, \eta_{p}^{2}=.02$. Pairwise comparisons of the within-subjects effect revealed that all groups reported a significant increase in level of worry following the negative emotion induction (all $p \mathrm{~s}<.001$ ). Follow-up pairwise tests of the interaction effect revealed that the GAD group $(\Delta M=2.57, S D=2.46)$ did not report a greater increase in worry following the emotion induction compared to the SAD group ( $\Delta M=2.17, S D$ $=2.01 ; p=.83)$ or NCC group $(\Delta M=1.85, S D=2.13, p=.57)$. Following the negative emotion induction, an increase in level of worry was associated with an increase in intensity of negative emotion $(r=.67, p<.001)$ and greater decrease in intensity of positive emotion $(r=-.26, p=$ $.04)$.

To examine changes in level of muscle tension/bodily arousal following the negative emotion induction, a repeated-measures ANOVA on muscle tension/ bodily arousal was 
performed with Time (Vanilla Baseline, imagery) as the within-subjects effect and Group (GAD, $\mathrm{SAD}, \mathrm{NCC})$ as the between-subjects effect. There was a main effect of Time, $F(1,61)=35.15, p$ $<.001, \eta_{p}^{2}=.37$, a main effect of Group, $F(2,61)=12.05, p<.001, \eta_{p}^{2}=.28$, but no Time $\mathrm{x}$ Group interaction, $F(2,61)=.43, p=.66, \eta_{p}^{2}=.02$. Follow-up comparison of the within-groups effect indicated a significant increase in level of muscle tension/bodily arousal from the vanilla baseline to following the negative emotion induction (all $p s<.01$ ). A posthoc comparison of the interaction effect indicated that the degree of change in muscle tension/bodily arousal was not different across the groups (all $p s>.05$ ). An increase in muscle tension/bodily arousal was associated with an increase in intensity of negative emotion following the negative emotion induction and $(r=.34, p=.01)$. There was no association between a change in muscle tension/bodily arousal and a change in the intensity of positive emotion $(r=-.17, p=.19)$ following the emotion induction. 
Objective 2b: Reactivity to the positive emotion induction. Table 8 displays the means and standard deviations for the measures of emotional reactivity to the positive emotion induction.

Vividness of mental imagery. A univariate ANOVA was conducted to examine group $(\mathrm{GAD}, \mathrm{SAD}, \mathrm{NCC})$ differences in vividness of mental imagery $(0=$ not at all vivid to $9=$ extremely vivid). There was a significant between-groups effect, $F(2,60)=4.73, p=.01, \eta_{p}^{2}=$ .14. Posthoc analyses showed that the SAD group reported significantly lower vividness $(M=$ $5.83, S D=1.70)$ than the GAD group $(M=7.00, S D=1.45 ; p=.04, d=0.74)$ and the NCC group $(M=7.15, S D=1.50 ; p=.02, d=0.82)$. There was no difference between the GAD group and NCC group $(p=.95, d=0.10)$. Correlation analyses were conducted to examine the association between imagery vividness and emotional reactivity. Lower vividness of mental imagery was significantly correlated with lower positive emotion reactivity $(r=-.55, p<.001)$, but was not correlated with negative emotion reactivity $(r=-.09, p=.51)$. Therefore, vividness was included as a covariate in subsequent analyses examining positive emotional reactivity to account for the significant association. 
Table 8

Emotional Reactivity to the Positive Emotion Induction

\begin{tabular}{|c|c|c|c|c|c|c|}
\hline & \multicolumn{2}{|c|}{$\begin{array}{l}\text { GAD } \\
n=20\end{array}$} & \multicolumn{2}{|c|}{$\begin{array}{c}\text { SAD } \\
n=23\end{array}$} & \multicolumn{2}{|c|}{$\begin{array}{c}\mathrm{NCC} \\
n=20\end{array}$} \\
\hline & Mean & SD & Mean & SD & Mean & SD \\
\hline \multicolumn{7}{|c|}{ Intensity of negative emotion } \\
\hline Vanilla baseline & 2.53 & 1.76 & 1.68 & 1.45 & 0.52 & 0.67 \\
\hline Imagery & 2.04 & 1.72 & 1.05 & 0.89 & 0.53 & 0.67 \\
\hline Change score & $-0.49^{\mathrm{a}}$ & 0.71 & $-0.63^{\mathrm{a}}$ & 0.84 & $0.01^{\mathrm{b}}$ & 0.40 \\
\hline \multicolumn{7}{|c|}{ Intensity of positive emotion } \\
\hline Vanilla baseline & 3.03 & 1.55 & 2.17 & 1.35 & 4.50 & 1.44 \\
\hline Imagery & 5.03 & 1.68 & 4.03 & 1.76 & 5.71 & 1.54 \\
\hline Change score & $1.99^{\mathrm{a}}$ & 1.90 & $1.86^{\mathrm{a}}$ & 1.63 & $1.21^{\mathrm{a}}$ & 1.15 \\
\hline \multicolumn{7}{|l|}{$\operatorname{RSA}^{\mathrm{c}}\left(\ln \mathrm{ms}^{2}\right)$} \\
\hline Vanilla baseline & 6.55 & 1.29 & 5.73 & 1.13 & 6.09 & 1.25 \\
\hline Imagery & 6.50 & 1.34 & 5.64 & 1.33 & 6.08 & 1.12 \\
\hline \multicolumn{7}{|l|}{ NS-SCR ${ }^{d}$ (frequency) } \\
\hline Vanilla baseline & 2.47 & 1.26 & 2.40 & 1.41 & 2.35 & 1.49 \\
\hline Imagery & 2.42 & 2.08 & 1.44 & 1.40 & 2.09 & 1.96 \\
\hline
\end{tabular}

Note. $\mathrm{GAD}=$ generalized anxiety disorder; $\mathrm{SAD}=$ social anxiety disorder $\mathrm{NCC}=$ nonclinical control; RSA = respiratory sinus arrhythmia; NS-SCR = mean frequency of nonspecific skin conductance responses per minute.

${ }^{a}$ Indicates a significant within-group effect from baseline to imagery.

${ }^{\mathrm{b}}$ Indicates significant difference from the GAD group

${ }^{\mathrm{c}}=\mathrm{GAD} n=21 ; \mathrm{SAD} n=21, \mathrm{HC} n=19$

${ }^{\mathrm{d}}=\operatorname{GAD} n=18 ; \operatorname{SAD} n=18, \operatorname{HC} n=15$ 
Subjective emotional reactivity. Regarding emotional reactivity to a positive emotion induction, it was hypothesized that the GAD group would report a greater increase in the intensity of positive emotion relative to the SAD group, but it was unknown whether the GAD group would differ from the NCC group on degree of emotional reactivity. In addition, it was hypothesized that participants would report a decrease in the intensity of negative emotion following the positive emotion induction task, however, no a priori hypotheses were made regarding differences in the magnitude of change in negative emotional intensity among the three groups.

To examine differences in positive emotional reactivity to the positive emotion induction, a repeated-measures ANOVA on subjective intensity of positive emotions following the positive emotion induction was performed with Time (Vanilla Baseline, Imagery) as the within-subjects effect and Group (GAD, SAD, NCC) as the between-subjects effect. There was a main effect of Time, $F(1,60)=70.13, p=<.001, \eta_{p}^{2}=.54$, and a main effect of Group, $F(2,60)=11.93, p=$ $<.001, \eta_{p}^{2}=.29$, but no Time x Group interaction, $F(2,60)=1.40, p=.27, \eta_{p}^{2}=.04$. Planned pairwise comparisons of the within-subjects effect revealed that all groups reported a significant increase in intensity of positive emotions following the positive emotion induction (all $p s<.001$ ). A follow-up test of the interaction effect revealed that the GAD group did differ in level of positive emotion reactivity compared to the $\operatorname{SAD}$ group $(p=.78 ; d=0.08)$ or the NCC group ( $p$ $=.13 ; d=0.50)$.

To account for the significant difference in level of vividness reported by the SAD group compared to the GAD and NCC groups, differences in positive emotional reactivity across the three groups were reanalyzed with vividness entered as a covariate. Consistent with the previous analysis, the follow-up test of the interaction effect revealed that the GAD group $\left(M_{\text {adjusted }}=1.80\right.$, 
$\mathrm{SE}=0.32)$ did not differ from the $\mathrm{SAD}$ group $\left(M_{\text {adjusted }}=2.26, \mathrm{SE}=0.31 ; p=.93, d=0.32\right)$ or the NCC group $\left(M_{\text {adjusted }}=0.94, \mathrm{SE}=0.32 ; p=.17, d=0.60\right)$ in level of positive emotional reactivity. However, different from the previous finding, when controlling for level of vividness, the $\mathrm{SAD}$ group was found to report greater positive emotional reactivity to the positive emotion induction relative to the NCC group ( $p=.02, d=0.92$ ).

To investigate differences in negative emotional reactivity to the positive emotion induction, a repeated-measures ANOVA was performed with Time (Vanilla Baseline, Imagery) as the within-subjects effect and Group (GAD, SAD, NCC) as the between-subjects effect. There was a main effect of Time, $F(1,60)=4.32, p<.001, \eta_{p}^{2}=.19$, and a main effect of Group $F(2$, $60)=10.54 p=<.001, \eta_{p}^{2}=.26$, and a significant Time $\mathrm{x}$ Group interaction, $F(2,60)=1.18, p=$ $.03, \eta_{p}^{2}=.12$. Planned pairwise comparisons of the within-subjects effect revealed that the GAD group ( $p=.01 ; d=0.28)$ and SAD group $(p<.001 ; d=.52)$ reported a significant decrease in negative emotion from baseline to following the positive emotion induction, but the NCC group did not report a significant change in negative emotion $(p=.96 ; d=0.01)$. Pairwise tests of the interaction effect showed that the GAD group reported a significantly greater decrease in negative emotion compared to the NCC group ( $p=.05, d=0.83$ ), but the GAD group did not differ from the SAD group $(p=.40, d=0.18)$.

Physiological reactivity. No known studies have examined parasympathetic responses to a positive emotion induction in people with GAD, therefore, it was unknown whether people with GAD would differ from people with SAD and people with no psychopathology on RSA following a positive emotion induction. A mixed model ANOVA on RSA was conducted with Group (GAD, SAD, NCC) as the between-subjects effect and Epoch ( 9 epochs, $30 \mathrm{~s}$ each) as the within-subjects effect. Mean RSA during the vanilla baseline prior to the positive emotion 
induction task was entered as a covariate. There was no main effect of Group, $F(2,55)=1.58, p$ $=.21$, and no Group $\mathrm{x}$ Epoch interaction, $F(16,440)=0.95, p=.52$. A priori pairwise comparisons of between-subjects effect indicated that the GAD group did not differ from the SAD group, $p=.93$, or the NCC group, $p=1.00$, in their RSA during the positive emotion induction.

In terms of sympathetic response, it was hypothesized that people with GAD would report equal levels of sympathetic reactivity compared to the NCC group but greater reactivity compared to the SAD group. A mixed model ANOVA on NS-SCR frequency was conducted with Group (GAD, SAD, NCC) as the between-subjects effect and Epoch (5 epochs, $60 \mathrm{~s}$ each) as the within-subjects effect. There was no main effect of group, $F(2,47.10)=0.77, p=.47$, and no Group x Epoch interaction, $F(8,46.01)=1.79, p=.10$. Planned pairwise comparisons of the between-subjects effect indicated that the GAD group did not differ from NCC group ( $p=1.00)$ or SAD group $(p=.07)$ on frequency of NS-SCRs during the positive mental imagery task.

Worry and muscle tension/bodily arousal reactivity. To examine changes in level of worry following the positive emotion induction, a repeated-measures ANOVA on worry was performed with Time (Vanilla baseline, Imagery) as the within-subjects factor and Group (GAD, $\mathrm{SAD}, \mathrm{NCC})$ as the between-subjects factor. There was a main effect of Group, $F(2,60)=14.05$, $p<.001, \eta_{p}^{2}=.32$, but no main effect of Time, $F(1,60)=0.93, p=.34, \eta_{p}^{2}=.02$, or Time $\mathrm{x}$ Group interaction, $F(2,60)=0.51, p=.61, \eta_{p}^{2}=.02$. Thus, the positive emotion induction did not result in a significant change in level of worry across the three groups. Change in worry was not associated with degree of change in positive or negative emotions during the positive emotion induction, $r=-.04, p=.77$ and $r=-.11, p=.40$, respectively. 
Next, to examine changes in level of muscle tension/bodily arousal, a repeated-measures ANOVA was performed with Time (Vanilla baseline, Imagery) as the within-subjects factor and Group (GAD, SAD, NCC) as the between-subjects factor. There was a main effect of Group, $F$ $(2,60)=7.93, p=.001, \eta_{p}^{2}=.21$, but no main effect of Time, $F(1,60)=1.42, p=.24, \eta_{p}^{2}=.02$ and no Time $\mathrm{x}$ Group interaction, $F(2,60)=1.54, p=.22, \eta_{p}^{2}=.05$. Thus, the positive emotion induction did not result in a significant change in level of muscle tension/bodily arousal across the three groups. Change in muscle tension/bodily arousal was also not associated with the degree of change in positive $(r=.01, p=.76)$ or negative $(r=-.04, p=.92)$ emotions during the positive emotion induction.

Summary of objective 2 findings. The second objective of the study was to examine whether people with GAD differ from people with SAD and people with no psychopathology on their level of emotional reactivity to two idiographic emotion inductions designed to elicit negative and positive emotions, respectively. People with GAD displayed significant subjective reactivity to the emotion inductions; however, the magnitude of their reactivity did not differ from the reactivity observed in the SAD group and the NCC group. People with GAD did not exhibit sympathetic or parasympathetic reactivity to the emotion induction tasks. Taken together, there appeared to be a discrepancy between subjective responses and objective responses to the emotion inductions in the GAD group. In addition, the GAD group was no more or less reactive to the emotion inductions relative to the SAD and NCC groups. 


\section{Discussion}

The emotion dysregulation model suggests people with GAD experience emotions at a heightened intensity relative to people with no psychopathology. Findings from cross-sectional, correlational research and laboratory experiments (e.g., Mennin, 2002, Mennin et al., 2005) provide preliminary support that individuals with elevated symptoms of GAD endorse experiencing emotions at a greater intensity relative to individuals with lower symptoms of GAD. To extend the extant research, the present study examined the subjective emotional and physiological responses of people with GAD while in a baseline, unmanipulated state and after an emotion induction task with the following aims: (1) to compare their level of baseline emotional intensity and level of emotional reactivity to that of people with SAD and people with no psychopathology and (2) to determine the extent to which their heightened intensity of emotional experiences can be attributed to greater intensity of baseline emotions and/or greater emotional reactivity.

\section{Trait Measures of Emotion and Emotion Regulation}

Regarding general intensity of emotional experiences, on the Affect Intensity Measure (AIM: Larsen \& Deiner, 1987), people with GAD endorsed experiencing intense emotions (irrespective of valence) at a greater frequency compared to people with no psychopathology, but with equivalent frequency as people with SAD. As discussed, scores on the AIM do not provide a measurement of the level of emotional intensity; therefore, a second self-report questionnaire was administered, the Emotional Intensity Scale (EIS; Bachorowski \& Braaten, 1994). Scores on the EIS provide a self-report assessment of average intensity of positive and negative emotions. On the EIS, people with GAD reported experiencing negative emotions at a greater intensity compared to both control groups. On the intensity of positive emotions, however, people with 
GAD did not differ from either comparison group. Taken together, the findings suggest that people with GAD report experiencing negative emotions at an elevated intensity, which is consistent with the findings from the preliminary tests of the emotion dysregulation model (e.g., Mennin et al., 2005; Mennin et al., 2009; Turk et al., 2005).

The finding that the three groups did not differ on self-reported intensity of positive emotions was inconsistent with the hypothesis. The experience of positive emotions has been less of a focus in anxiety disorders, as past theories often suggested that anxiety is associated with greater negative emotions, but is unrelated to positive emotions (Watson, Clark, \& Carey, 1988). However, subsequent studies have reported that positive emotion, and particularly the experience of lower positive emotion, may be present in individuals with anxiety disorders. Although the findings regarding the intensity of positive emotion experienced by people with GAD remains equivocal, there is greater evidence to suggest that elevated symptoms of GAD are associated with greater fear of positive emotions and lower expression of positive emotions (Mennin et al., 2005; Roemer et al., 2005; Turk et al., 2005). Alternatively, the findings related to social anxiety are more conclusive; people with social anxiety are consistently found to report lower intensity of positive emotion relative to people with no psychopathology (e.g., Kashdan, 2007; Watson et al., 1988). As a result, it was noteworthy that there was no difference between people with SAD and people with no psychopathology on their reported intensity of positive emotion. It is possible that the self-report questionnaire used in the present study may account for the inconsistency with findings from past research. No known studies have compared people with GAD, or people with SAD, to people with no psychopathology on the EIS, which asks people to report on the intensity of emotion that they would experience in a variety of everyday scenarios (Bachorowski \& Braaten, 1994). 
Findings from the self-report questionnaires of emotion regulation suggest that people with GAD experience significantly greater difficulty with regulating their emotional experiences, defined as lower clarity of emotional experiences, nonacceptance of emotions, inability to engage in goal-directed behavior and refrain from impulsive behavior when distressed, and greater use of ineffective emotion regulation strategies relative to people with no psychopathology. One area of emotion regulation for which people with GAD did not report difficulties was their level of emotional awareness. This finding was consistent with past research in nonclinical samples (Novick-Kline et al., 2003; Salters-Pedneault, Roemer, Tull, Rucker, \& Mennin, 2006), and suggests that people with GAD report equivalent awareness of their emotional experiences relative to people without GAD. Related to the use of different emotion regulation strategies, people with GAD reported lower use of cognitive reappraisal compared to people with no psychopathology, but the two groups did not differ on their use of emotional suppression. Greater use of cognitive reappraisal has been associated with reduced negative affect (Gross, 1998a); therefore, the lower reported use of cognitive reappraisal as an emotion regulation strategy by people with GAD may have a role in maintaining their negative emotions. Finally, there were no differences found for people with GAD relative to those with SAD in their difficulties with emotion regulation or use of emotion regulation strategies, which is consistent with the proposal that difficulties with emotion management may be present across the anxiety disorders (Cisler, Olatunji, Feldner, \& Forsyth, 2010).

In summary, the findings based on participants' responses to trait measures of emotional experiences and emotion dysregulation were in accordance with the arguments put forward by the emotion dysregulation model (Mennin et al., 2005); that is, people with GAD exhibit heightened intensity of negative emotions and greater emotion dysregulation relative to people 
with no psychopathology. Moreover, when compared to people with SAD, the experience of heightened intensity of negative emotions appears to be specific to people with GAD (Mennin et al., 2009), but both groups report difficulty with managing their emotional experiences. Given that the findings on trait self-report measures do not distinguish between baseline emotional intensity and emotional reactivity, the next objectives of the study were to compare the three groups on their baseline emotional intensity and immediate reactivity to emotionally evocative stimuli.

\section{Baseline Emotional Intensity}

Subjective emotional intensity rating. Prior examinations of emotional intensity in people with GAD have relied on trait self-report questionnaires as an indication of their typical intensity of emotions. However, it is unknown whether these findings reflect the intensity of baseline emotions, operationalized as emotional experiences that occur independent of direct exposure to an external stimulus (Jacob et al., 1999). Therefore, to extend prior research, the present study examined participants' subjective intensity ratings of current emotion while in an unmanipulated, baseline state. Differences in the intensity of negatively- and positively-valenced emotions were examined independently.

As hypothesized, people with GAD reported significantly greater subjective intensity of their negative emotions at baseline relative to those of people with SAD and people with no psychopathology. It is of note that people with SAD also reported significantly greater intensity of negative emotions compared to those of people with no psychopathology, but to a lesser degree relative to people with GAD. The finding that people with GAD report elevated intensity of negative emotions is largely consistent with the emotion dysregulation model (e.g., Mennin et al., 2005; Mennin et al., 2007; Turk et al, 2005). Moreover, the baseline subjective intensity 
results are consistent with participants' responses to the trait, self-report measures, as well as previous examinations of state, baseline emotional experiences reported by analogue GAD samples when compared to nonclinical control group (e.g., Llera \& Newman, 2014; Mennin et al., 2005).

The hypothesis that people with GAD would report lower subjective intensity of positive emotions while in a baseline, unmanipulated state compared to those of people with no psychopathology, but greater intensity of positive emotions compared to people with SAD, was partially supported. People with GAD reported lower intensity of positive emotions compared to people with no psychopathology, but there was no difference between the two clinical groups. The finding that people with GAD reported lower subjective intensity of positive emotions relative to people with no psychopathology is consistent with a recent study by Llera and Newman (2014), who found that that people with elevated symptoms of GAD report experiencing lower contentment and happiness when they were in a neutral state compared to nonanxious controls.

It is to be noted that in the present study, differences among the three groups in reported intensity of positive emotions on the trait questionnaires were inconsistent with participants' reported intensity on state measures. On the trait self-report measure, the EIS, there was no difference across the three groups in their reported intensity of positive emotions, whereas on the baseline, state measure of emotional intensity, people with GAD and people with SAD reported lower intensity of positive emotions relative to people with no psychopathology. Similar discrepancies between state and trait measures of emotional intensity are noted elsewhere in the literature; that is, on trait questionnaires, people with GAD and people with no psychopathology report equal levels of intensity of positive emotion (Mennin et al., 2005; Turk et al., 2005), but 
on state measures people with GAD report lower intensity of positive emotion (Llera \& Newman, 2014). It is possible that the discrepancy may be accounted for by the different elements of a construct that are assessed by state and trait measures. For example, when assessing negative emotionality, the trait measure reflects dispositional, or average, negative emotional states, whereas the state measure reflects negative emotion that is present "right now" (Watson et al., 1988). As a result, trait and state measures are not always highly correlated.

Moreover, various internal and external factors may influence people's responses on state versus trait questionnaires. For example, trait measures may be affected by retrospective recall bias (Solhan, Trull, Jahng, \& Wood, 2009) to a greater extent than state measures, whereas, state measures may be biased by situational contexts. Thus, neither state nor trait measures may be a true representation of "average" emotional intensity. To account for these limitations, future studies may benefit from the use of experience sampling method (ESM), as multiple assessments of state emotional intensity can be averaged to provide an alternative measure of trait emotional intensity (Diener \& Emmons, 1984; Stone \& Shiffman, 1994). Trait emotional intensity calculated via this method would likely provide an assessment that is less affected by retrospective bias and would also capture the natural variability in state emotional intensity (Diener \& Emmons, 1984; Solhan et al., 2009). No known studies have compared the responses provided by people with GAD on trait self-report questionnaires to those from an experience sampling study; however, significant discrepancies have been found on emotional instability assessments via ESM and self-report questionnaires in individuals with borderline personality disorder and individuals with major depressive disorder (Solhan et al., 2009).

Subjective rating of worry and muscle tension. In addition to subjective emotional intensity, the present study investigated the extent to which individuals with GAD differ on their 
subjective ratings of level of worry and level of muscle tension/bodily arousal when in a baseline, unmanipulated state compared to people with SAD and people with no psychopathology. As expected, people with GAD reported greater levels of worry and muscle tension/bodily arousal relative to people with no psychopathology, but did not differ from people with SAD. Thus, in addition to greater negative emotional intensity, people with GAD also reported greater worry and associated subjective muscle tension/bodily arousal when in a baseline state.

The relationship between baseline subjective emotional intensity and reported level of worry and level of muscle tension/bodily arousal was examined further. Greater worry and greater muscle tension/bodily arousal were associated with significantly greater intensity of negative emotions and lower intensity of positive emotions. It is of note that when controlling for the variance accounted for by worry and muscle tension/bodily arousal, the difference in baseline intensity of negative emotions between people with GAD and people with SAD was no longer significant. Therefore, severity of worry and muscle tension/bodily arousal may have a role in exacerbating the intensity of negative emotions experienced by people with GAD. Indeed, past research has noted that worry is associated with an increase in negative emotion (e.g., Llera \& Newman, 2010); however, it was not possible to examine the causal associations in the present study.

Physiological response. In addition to subjective features of emotion, physiological response may also be informative for understanding emotion (e.g., Lang et al., 1998). Therefore, the present study also incorporated assessments of the sympathetic (assessed via NS-SCRs) and parasympathetic (assessed via RSA) nervous systems, as both branches of the autonomic nervous system have a role in emotional arousal (Bradley \& Lang, 2007). Regarding baseline 
sympathetic arousal, the hypothesis that people with GAD would exhibit similar levels of sympathetic arousal at baseline relative to people with SAD and people with no psychopathology was supported. There was no difference in the frequency of NS-SCRs at baseline across the three groups, which was consistent with previous studies examining baseline sympathetic arousal in people with GAD (e.g., Fisher et al., 2009).

In contrast, the prediction that people with GAD would exhibit lower resting RSA relative to people with no psychopathology was not supported. That is, there were no differences among the three groups in resting vagal tone. The extant findings on parasympathetic reactivity in people with GAD relative to people with no psychopathology is mixed. For example, during a laboratory experiment, resting RSA values in people with GAD were found to be lower than those of nonclinical controls (e.g., Thayer et al., 1996). Alternatively, results from an ambulatory study found no differences between people with GAD and nonclinical controls on resting heart rate (Hoehn-Saric et al., 2004). Therefore, additional research is needed to clarify the role of parasympathetic arousal in people with GAD. Furthermore, it may be more informative to examine a different measure of autonomic arousal, such as muscle tension, in people with GAD.

Taken together, findings highlight a discrepancy between people's baseline subjective emotional arousal and physiological response. Although people with GAD reported greater subjective emotional intensity, greater levels of worry, and greater muscle tension/bodily arousal relative to the two comparison groups, their autonomic response was not reflective of these differences. The discrepancy between subjective and physiological emotional arousal has been noted previously in the GAD literature and one explanation put forward to account for the discrepancy is that people with GAD exhibit greater physiological inflexibility (e.g., Borkovec \& Hu, 1990; Hoehn-Saric \& McLeod, 2000). That is, people with GAD are reported to maintain 
steady, but elevated, physiological arousal relative to people with no psychopathology and therefore, exhibit lower physiological reactivity to laboratory stressors relative to nonanxious controls (Hoehn-Saric \& McLeod, 2000). The discrepancy between subjective and physiological states is examined further later in the discussion.

\section{Emotional Reactivity}

The present study also sought to investigate the degree to which people with GAD exhibit greater emotional reactivity to negative and positive emotion inductions in comparison to people with SAD and people with no psychopathology. Emotional reactivity was defined as the difference between emotional intensity prior to and following each emotion induction as assessed by participants' subjective and physiological response (Kuo \& Linehan, 2009).

\section{Negative emotion induction.}

Subjective emotional reactivity. The hypothesis that people with GAD would exhibit greater subjective emotional reactivity to a negative emotion induction compared to people with SAD and people with no psychopathology was not supported. Although individuals with GAD reported an increase in negative emotion and a decrease in positive emotions after the negative emotion induction (i.e., mental imagery of a self-identified worst case scenario), the degree of emotional reactivity was equivalent to that of people with SAD and people with no psychopathology. It is of note that people with SAD also did not differ from people with no psychopathology on their degree of subjective emotional reactivity to the negative emotion induction.

The lack of heightened subjective emotional reactivity in people with GAD is inconsistent with findings from a previous study which showed that individuals with elevated symptoms of GAD exhibited greater emotional reactivity to a negative emotion induction 
relative to people with no psychopathology (Mennin et al., 2005). It is noteworthy that the present study used a different type of emotion induction task and different measure to assess subjective emotional response. That is, Mennin and colleagues (2005) used a music clip to induce negative emotion, whereas an idiographic script of a worst case scenario was used in the present study. It is surprising that the use of a nonpersonalized, standard emotion music induction resulted in greater emotional reactivity than the idiographic emotion induction, as Ellard, Farchione, and Barlow (2012) reported that emotion inductions (i.e., film clips, images and music clips) that incorporate greater personal relevance are associated with greater emotional reactivity. However, in their review of different emotion inductions, Ellard and colleagues did not compare the effect of a mental imagery script to that of a music clip.

Although mental imagery, or guided imagery, is a useful method to evoke emotion (Lang et al., 1983; Pitman et al., 1987), it is possible that people with GAD exhibit different degrees of emotional reactivity based on the type of emotion induction used. In addition, there may be other factors related to the emotion induction task that may have attenuated the degree of emotional reactivity, which are explored later in the discussion. Finally, although heightened emotional reactivity is inferred by the emotion dysregulation model (e.g., Mennin et al., 2005), it is possible that the people with GAD do not exhibit greater emotional reactivity relative to people with SAD and people with no psychopathology, as the results suggest.

Physiological reactivity. In terms of physiological reactivity to the negative emotion induction, it was hypothesized that people with GAD would exhibit greater physiological rigidity, as evidenced by lower RSA reactivity and fewer NS-SCRs, in comparison to people with SAD and people with no psychopathology. Following the emotion induction, people with GAD displayed a degree of physiological rigidity as evidenced by an absence of parasympathetic 
and sympathetic reactivity. Inconsistent with the study hypothesis, however, none of the groups displayed sympathetic or parasympathetic reactivity to the negative emotion induction.

The lack of physiological reactivity for people with GAD is consistent with past research indicating that people with GAD have rigid autonomic responses (e.g., Kreibig, 2010; Lyonfields et al., 1995). Moreover, the discrepancy between people's self-reported change in physical tension and arousal relative to their sympathetic response is also in line with previous findings. That is, people with GAD are found to subjectively report changes in bodily arousal (i.e., increased heart rate and sweatiness) that are not consistent with concurrent physiological measures of heart rate and electrodermal activity (e.g., Hoehen-Saric \& MacLeod, 2000). Moreover, outside of GAD research, there is ample research supporting significant discrepancies in subjective versus physiological reactivity in response to fear inductions (e.g., Cook et al., 1988; Lang, 1968; Marks \& Huson, 1973; McTeague \& Lang, 2012). However, the lack of sympathetic and parasympathetic response reported by people with no psychopathology was noteworthy and inconsistent with past research (Larsen, Berntson, Poehlmann, Ito, \& Cacioppo, 2008), which suggests that perhaps the results should be interpreted with caution.

The emotion system is made up of multiple response systems, including cognitive, physiological and motor responses (Lang, 1971). Despite the fact that all of these responses are controlled and influenced by the brain, the degree of reactivity across the response systems to the same stimulus may vary and to a certain degree, may be independent of the other systems (Lang, 1968). Therefore, although participants reported an increase in subjective emotional intensity, the same degree of emotional reactivity as assessed via their physiological responses may not be present. In a seminal paper, Hodgson and Rachman (1974) hypothesized that when emotional arousal is stronger (versus milder) and a task is less (versus more) demanding, the likelihood that 
there will be concordance between emotional response systems is greater. With this in mind, it is possible that if the idiographic emotion induction task was not stressful enough or perhaps was too cognitively demanding, it may have resulted in the discordance between subjective and physiological responses. Furthermore, people who report having greater ability to form vivid mental imagery are also found to exhibit greater emotional reactivity when asked to imagine a scenario (Miller et al., 1987). Although the three groups did not differ in their reported vividness of their mental imagery during the emotion induction task, it is possible that across the groups, participants may have had difficulty imagining the scenarios, which may have attenuated their physiological reactivity.

Finally, it is possible that discordance between people's subjective and physiological arousal may be reflective of a misperception of their bodily states. For example, following the negative emotion induction, participants reported a significant increase in muscle tension/body arousal, however, this was not reflected in their autonomic responses. Moreover, people's perception of their bodily state may be strongly influenced by their expectations (McLeod \& Hoehn-Saric, 1993; McLeod, Hoehn-Saric \& Stefan, 1986). Therefore, participants may have expected to experience greater physiological response following imagining a stressful event and therefore, reported greater subjective emotional and bodily arousal.

\section{Positive emotion induction.}

Subjective reactivity. A unique component of the present study was the examination of how people with GAD react to a positive emotion induction, which required people to think about an upcoming pleasant situation. Understanding reactivity, or lack of reactivity, to positive events is informative, as the experience of positive emotion has important mental and physical health implications and affects people's resilience to stress (e.g., Fredrickson, 1998; Tugade \& 
Fredrickson, 2004). Based on prior, albeit limited, findings it was hypothesized that people with GAD would report equivalent levels of reactivity compared to people with no psychopathology, but greater positive emotional reactivity compared to people with SAD. The hypothesis was partially supported. People with GAD showed a significant increase in their positive emotions and a decrease in their negative emotions following the positive emotion induction, however, the degree of reactivity did not differ from people with no psychopathology or from people with SAD.

In addition, people with SAD also reported a significant increase in their subjective intensity of positive emotions and decrease in negative emotions following the positive emotion induction. This finding is inconsistent with previous research suggesting that people with SAD have blunted or adverse reactions to positive experiences (Kashdan \& Steger, 2006; Turk et al., 2005), which would suggest a lack of positive emotional reactivity. As discussed in the negative emotion induction, participants across the three groups reported equivalent levels of vividness and engagement during the emotion induction task. Regarding the positive emotion induction task, however, people with SAD reported significantly lower vividness of their mental imagery as well as lower engagement in positive mental imagery. When controlling for the variance accounted by the vividness of mental imagery in a person's level of reactivity to the positive emotion induction, people with SAD were found to have greater reactivity to the positive emotion induction relative to people with no psychopathology. Therefore, it is possible that people with SAD avoided forming vivid mental imagery, possibly consciously or unconsciously, which may have impacted their emotional reactivity to the task.

Physiological reactivity. A limited number of studies have examined the physiological reactivity exhibited by people with GAD following a positive emotional experience; therefore, 
an aim of the present study was to add to the existing literature by examining how people respond physiologically to a positive emotion induction. The results showed that people with GAD did not differ from people with SAD or people with no psychopathology in terms of their physiological reactivity to the positive emotion induction. A lack of reactivity was also noted in their subjective responses of muscle tension and bodily arousal; that is, none of the groups reported a significant change in their level of muscle tension/bodily arousal following the positive emotion induction.

Overall, the lack of physiological arousal following a positive emotion induction in people with GAD, as well as those with no psychopathology, was inconsistent with previous findings. For example, Llera and Newman (2014) reported that from a baseline worried, neutral or relaxed state, both people with analogue GAD and nonanxious controls reported an increase in sympathetic arousal (i.e., greater amplitude of their NS-SCRs) while watching a humourous video clip. As discussed, given the association between mental imagery ability and physiological reactivity, it is possible that the mental imagery evoked by the tasks was not sufficiently vivid (Miller et al., 1987) or evocative of salient emotions (Hodgson \& Rachman, 1974) to lead to a physiological response.

\section{Emotion Dysregulation Model}

The primary aim of the study was to add to the literature on emotion dysregulation in people with GAD by clarifying whether the construct of heightened emotional intensity is related to a heightened baseline emotional intensity and/or emotional reactivity. Taken together, the findings suggest that for people with GAD, the subjective experience of elevated baseline emotional intensity, not degree of emotional reactivity, accounts for the differences in heightened emotional intensity relative to people with no psychopathology and people with SAD. Moreover, 
the findings show that elevated negative emotional intensity is not solely the result of having an anxiety disorder, but may be specific to GAD.

It should be noted that the subjective mean intensity rating of baseline negative emotion reported by people with GAD was only 2.30 on the scale from 0 (not at all) to 8 (extremely; range $=0.50$ to 4.67$)$. As this rating is on the lower end of the emotional intensity continuum, it may be argued that their mean negative emotional intensity rating does not provide compelling evidence that they experience heightened emotional intensity. However, although not at an extreme level, the relative elevation in intensity of negative emotion found in people with GAD compared to people with no psychopathology may be associated with lower psychological wellbeing and greater difficulty modulating emotional experiences (Mennin et al., 2005).

To examine the possible negative impact of elevated intensity of negative emotion, posthoc analyses were performed to examine the relationship between participants' baseline emotional intensity and self-reported difficulty with emotion regulation. Indeed, it was found that greater intensity of negative emotions was associated with greater difficulty with emotion regulation $(r=.58, p<.001)$, lower use of adaptive emotion regulation strategies (e.g., cognitive reappraisal; $r=-.26, p=.04)$, greater levels of worry $(r=.72, p<.001)$, greater muscle tension/bodily arousal $(r=.57, p<.001)$, higher state anxiety $(r=.65, p<.001)$ and stronger symptoms of depression $(r=.52, p<.001)$. Moreover, greater negative emotions were also associated with lower intensity of positive emotions $(r=-.25, p=.05)$. In sum, irrespective of whether there is evidence that people with GAD experience heightened intensity of negative emotions, even moderately intense negative emotions may be detrimental to people's overall mood and ability to manage emotional experiences effectively, which is proposed by the emotion dysregulation model (Mennin, 2002; Mennin et al., 2005). A longitudinal assessment of 
emotional experiences would assist in delineating the causal relationship between greater intensity of negative emotions and the factors listed above, and would also clarify whether people with GAD consistently report elevated intensity of negative emotions relative to people no psychopathology.

In terms of emotional reactivity, the findings suggest that people with GAD report the same degree of emotional reactivity to stimuli (positive and negative), as people with social anxiety and people with no psychopathology. As noted, this finding was inconsistent with other experiments examining differences in emotional reactivity in an analogue sample of people with GAD relative to people with no elevated symptoms of GAD (e.g., Mennin et al., 2005). To better understand emotional reactivity in people with GAD, research studies outside of the laboratory, for example daily diary or experience sampling studies, may provide a better understanding of how people with GAD react to negative events or stimuli in their environment. Moreover, although people with GAD did not exhibit heightened emotional reactivity relative to the control groups, given that they report heightened baseline emotional intensity, any increase in negative emotions following a negative event may have detrimental consequences and lead to greater difficulty with effective emotion regulation.

Taken together, the findings show that baseline emotional intensity, and not level of emotional reactivity, may account for the differences in emotional intensity reported by people with GAD relative to people with SAD or people with no psychopathology. Similar results have been found for people with borderline personality disorder (BPD), as people with BPD are found to exhibit greater baseline emotional intensity, but not greater levels of emotional reactivity when compared to people with SAD and people with no psychopathology (Kuo \& Linehan, 2009). Future research would benefit from examining the factors that cause people with GAD to 
experience elevated negative emotions and the extent to which this emotional pattern is a risk factor for the development of GAD or a consequence of the symptoms associated with GAD.

\section{Strengths, Limitations and Future Directions}

The present study addressed several limitations of the previous tests of the emotion dysregulation model by including the use of a clinical GAD sample, a clinical comparison group, and idiographic emotion inductions to better understand the emotion generative process in people with GAD, however, there are also limitations to consider.

The present study adds to the extant literature by comparing a clinical sample of people with GAD to a clinical sample of people with SAD. Prior tests of the emotion dysregulation model have included people with SAD as a comparison group, however, these tests were primarily conducted in analogue samples and with the use of trait self-report measures. As hypothesized, and consistent with past findings (e.g., Mennin et al., 2007; Turk et al., 2005), people with GAD reported greater intensity of their negative emotions compared to people with SAD. There was no difference in their reported subjective intensity of positive emotion, however, the findings were in the predicted direction; people with SAD reported lower intensity of positive emotion relative to people with GAD. Taken together, the findings suggest that there may be some distinctions between people with GAD and people with SAD in their experiences of positive and negative emotions, but similarities in their overall difficulty with engaging in adaptive emotion regulation (Cisler et al., 2010; Turk et al., 2005). As highlighted, emotions are dynamic and as a result, people engage in various emotion regulation strategies to meet the demands of their environment, which is not always captured via single assessments of state or trait emotional intensity (Stone \& Shiffman, 1994). Thus, future comparisons of emotional experiences and emotion regulation in people with GAD compared to people with SAD may 
benefit from the use of longitudinal assessment methods, including experience sampling.

In addition, a strength of the study was the use of an idiographic emotion induction task. A semistructured emotion induction script was chosen to maximize both internal and external validity. Participants chose the content of the script, including which personally-relevant positive and negative event they were to imagine (e.g., presentation that was not going well; hearing that a loved one's cancer had returned). To standardize and ensure a level of consistency across the scripts, participants were required to provide the same descriptive features (i.e., what they would hear, smell, feel, and see) and to select from a list of adjectives to describe their emotional and physiological responses to the event (adapted from Kuo \& Linehan, 2009; Pitman et al., 1987; Yamasaki, 2006). Although this method increased internal and external validity of the task, it is possible that by having participants develop their own emotion induction script, it may have contributed to the inconsistent findings compared to Mennin et al. (2005).

Moreover, a limitation of the emotion induction task, is that unlike previous studies where participants were unaware of the content of the emotion induction prior to engaging in the task, participants in the present study had exposure to the content of the emotion induction task during the generation of their scripts. Therefore, the scenario may have been less activating or emotionally evocative during the emotion induction task because participants were already primed to the material. Literature on exposure to mental imagery scripts of feared scenarios suggests that repeated exposure to the same scenario is associated with reduced emotional arousal (Fracalanza, Koerner, \& Antony, 2014). As a result, listening to the audio recorded script during the emotion induction task may not have had the same emotional effect compared to discussing the scenario for the first time. Participants were not monitored for subjective or physiological arousal during the script development; however, this may be beneficial in future 
studies. Moreover, to overcome the limitations related to the script development, future studies may ask participants to select from generic scripts that match their worry themes. The use of generic scripts may still be activating enough because the topic would still target the individuals' fear structure (Huppert \& Foa, 2004), and a benefit is that participants would not be exposed to the content of the emotion induction prior to engaging in the task itself.

As noted, participants were not monitored during the script development procedure, so the level of emotional arousal experienced while developing the script is unknown. If the discussion of the worrisome scenario was aversive to participants during the development of their emotion induction scripts, they may not have fully participated in the actual emotion induction task in an attempt to avoid emotional, and possibly physiological, reactivation. Thus, being aware of the content of what they were required to imagine ahead of time may have negatively affected participants' engagement with the emotion induction task and their subsequent level of reactivity. Although there were no significant differences across the three groups on their reported level of engagement in mental imagery, a closer inspection of the mean score suggests that participants in all three groups may have experienced some difficulty with fully engaging in and focusing on the required mental imagery, as well as engaged in some use of distraction. Therefore, it is possible that low engagement with the task may have attenuated participants' subjective and physiological reactions to the emotion induction tasks.

Furthermore, it is possible that demand effects may have a role in the discrepancy between participants' subjective self-report and physiological response. Westermann, Spies, Stahl, and Hesse (1996) argue that mood inductions are at risk of being biased because participants' responses reflect their expected experience rather than their actual experience. Therefore, additional research is needed to clarify why there was a discrepancy between 
subjective and physiological reactivity and the possible role of perceptual biases.

Finally, it is unknown if the findings from the present study would correspond with people's subjective and physiological reactions to positive or negative events in daily life. Perhaps outside of the laboratory context, greater subjective and physiological reactivity would be observed when people with GAD encounter positive or negative life events. Future studies would benefit from investigating reactivity to positive or negative situations in people's daily lives with the use of the experience sampling method (Hektner, Schmidt, \& Csikszentmihalyi, 2007).

\section{Summary and Conclusion}

The present study examined the subjective emotional and physiological responses of people with GAD while in a baseline, unmanipulated state and immediately following emotionally evocative stimuli, to examine the extent to which greater intensity of emotional experiences can be attributed to heightened intensity of baseline emotions and/or greater emotional reactivity. A second aim was to investigate whether the emotional experiences of people with GAD differ from people with SAD and people with no psychopathology. Overall, people with GAD were found to have greater subjective emotional intensity at a baseline, unmanipulated state relative to people with SAD and to people with no psychopathology, but did not exhibit greater emotional reactivity to emotionally-evocative events. Given that the absolute level of emotional intensity exhibited by people with GAD was low, additional research should further explore the extent to which people with GAD exhibit heightened intensity of negative emotions as described by the emotion dysregulation model of GAD. 


\section{Chapter 3: An Experience Sampling Study of Emotional Experiences in the Daily Life of People with Generalized Anxiety Disorder}

A growing body of research suggests that difficulties with emotion and emotion regulation have a significant role in the development and maintenance of generalized anxiety disorder (GAD). To better understand this relationship, Mennin and colleagues (2005) proposed the emotion dysregulation model of GAD. Empirical tests of the model suggest that there are two main factors that contribute to emotion dysfunction in people with GAD: experiencing emotions at a heightened intensity and engaging in maladaptive emotion regulation strategies when experiencing distress. Moreover, Mennin argues that worry is one of the maladaptive coping strategies that people with GAD use in an attempt to attenuate or control their emotional experiences (Mennin et al., 2005).

To date, most of our knowledge on the generation and regulation of emotion in people with GAD is based on cross-sectional correlational studies and laboratory experiments; therefore, the extent to which these findings generalize to their everyday emotional experiences is unknown. Moreover, given that emotional experiences fluctuate over time, there are limitations to how accurately cross-sectional designs and once-daily diary assessments can describe dynamic experiences. Momentary examinations of people's emotions via experience sampling methodology (ESM), however, can provide more nuanced details about people's everyday emotional experiences and their patterns of reactivity to events in daily life (Ebner-Priemer \& Trull, 2009). In ESM, participants provide multiple, real-time assessments of their experiences (Csikszentmihalyi \& Larson, 1987) and as a result, ESM has greater accuracy and reliability and is less susceptible to memory biases than are retrospective self-report measures and once-daily diaries (e.g., recording experiences from the day each evening). Computerized ESM (e.g., 
entering responses on a device) also has an added advantage over paper diaries because response times are recorded automatically, which provides a check of participants' level of compliance with the protocol and discourages "backfilling" (Feldman Barratt \& Barratt, 2001). Taken together, the use of ESM may enhance the study of emotional experiences in people with GAD.

Therefore, to better understand the emotional experiences of people with GAD, the present study had three primary aims: first, to examine the extent to which people with GAD differ from people with no psychopathology on their experience of negative and positive emotions in daily life. Specifically, to what extent do people with GAD exhibit differences in the intensity, instability, and inertia of emotions compared to people with no psychopathology. Second, to determine the extent to which engaging in worry directly affects the emotional experiences of people with GAD compared to people with no psychopathology. Third, to investigate the extent to which people with GAD exhibit greater emotional reactivity following self-appraised negative and positive events in daily life.

\section{Intensity, Instability, and Inertia of Emotions}

Although people with GAD are reported to have disturbances in their emotional experiences (Brown, Chorpita, \& Barlow, 1998), little is known about the patterns of emotional experiences in their daily lives. Moreover, it is unknown whether the emotional patterns of people with GAD differ from those of people with no psychopathology. Much of the research studying the emotional experiences of people with GAD has relied on retrospective self-reports in cross-sectional studies, however, as noted, this study design cannot adequately capture the "ebb and flow" of emotional experiences in daily life (Ebner-Priemer \& Trull, 2009). In addition, several researchers highlight discrepancies in findings from studies using retrospective selfreport compared to real-time assessments (e.g., Solhan et al., 2009; Stone et al., 1998; Trull \& 
Ebner-Priemer, 2009). For example, when comparing retrospective and daily reports of negative affect by people with depression and people without depression, Bylsma, Taylor-Clift, and Rottenberg (2011) found that both groups reported less negative affect retrospectively compared to real-time assessments. The lack of concordance between the retrospective and momentary assessments described by people with depression provides further support to examine the realtime emotional experiences in people with GAD.

Only one known diary study has compared the emotional experiences of undergraduate students with elevated or low symptoms of GAD using a once-a-day diary (Decker et al., 2008). At the end of each day, for 8 days, participants described their most intense emotional experience of the day. On days when negative emotions were the dominant emotion, people with GAD reported experiencing negative emotions at a greater intensity compared to people without GAD. There were no differences between the two groups on their reported intensity of positive emotions, when positive emotions were the dominant emotion of the day.

The findings by Decker et al. (2008) highlight how people with GAD differ in their emotional experiences, and emotional intensity, compared to people without GAD; however, there are several limitations to this study. First, participants provided emotion ratings only at the end of each day. As a result, the diary entries were limited by the potential for retrospective memory bias (Stone et al., 1998). Second, participants were asked to report on only one emotional experience each day, regardless of how many emotions were experienced over the course of the day. Alternative methods, such as ESM, would minimize memory biases and provide more nuanced details by asking people to rate the intensity of several emotions in the moment (Trull \& Ebner-Priemer, 2009). Therefore, to contribute to the GAD literature, the present study examined the extent to which people with GAD differ from people with no 
psychopathology in the dynamic nature of their emotions, including the instability and inertia of their emotional responses.

Instability of emotional experiences is defined as the degree of change in the intensity of emotion from one moment to the next and is measured by averaging the differences between successive squared emotion intensity ratings (i.e., mean squared successive differences, MSSD; Jahng, Wood, \& Trull, 2008). Unlike the assessment of emotional variability, which reflects how much people's emotional experiences deviate from their average emotional state (i.e., withinperson standard deviation; Eid \& Diener, 1999; Kuppens, Allen, \& Sheeber, 2010), instability accounts for both the variability and the temporal dependency between two successive emotion intensity ratings (Ebner-Priemer, Eid, Kleindienst, Stabenow, \& Trull, 2009; Jahng et al., 2008). Greater instability has been associated with neuroticism (e.g., Murray, Allen, \& Trinder, 2002), depression (e.g., Hall, Sing, \& Romanowski, 1991; McConville \& Cooper, 1996), and borderline personality disorder (e.g., Stein, 1996). Thus, the present study aims to examine whether greater instability is also present in people with GAD.

Inertia of emotional experiences is operationalized as the autocorrelation of people's current level of emotional intensity with their emotional intensity at the previous time point (Kuppens et al., 2010; Suls, Green, \& Hillis, 1998). Greater emotional inertia is analogous to being "stuck" in an emotional experience (Koval et al., 2012) and the persistence of an emotion for an extended duration may be related to difficulties with emotion regulation (Koval \& Kuppens, 2012). Taken together, determining the instability and level of inertia provides a clearer picture of how the emotions that people with GAD experience fluctuate in daily life. For example, a profile of high instability and high inertia would be suggestive of large, but slow shifts in emotional experiences, whereas a profile of high instability, but low inertia would be 
suggestive of large and fast shifts (Houben, Van Den Noortgate, \& Kuppens, 2015; Koval, Pe, Meers, \& Kuppens, 2013). To date, no known studies have examined the extent to which the daily emotional experiences of people with GAD show greater instability or inertia compared to those of people with no psychopathology. Therefore, the present study makes an important contribution to the literature on emotion dynamics in people with GAD.

\section{Emotional Reactivity to Worry}

Worry is a part of everyday life regardless of whether a person has a diagnosis of GAD (Dupuy, Beaudoin, Rhéaume, Ladouceur, \& Dugas, 2001). Thus, it is important to understand how worry affects people's emotional experiences and the extent to which this differs for people with GAD compared to people without GAD. Several theoretical models of GAD posit that worry affects people's emotional experiences; however, how worry affects people's emotional experiences differs across the models.

The avoidance theory of worry (Borkovec et al., 2004), for example, suggests that people engage in worry (defined as a primarily verbal thought process) to inhibit the formation of negative mental imagery and the subsequent experience of negative emotions. Alternatively, the emotion dysregulation model (Mennin et al., 2005) argues that people with GAD experience negative emotions as aversive and therefore engage in worry as a maladaptive strategy to cope with their emotional experience. More recently, Newman and Llera (2011) proposed the contrast avoidance model, which suggests that people engage in worry not to attenuate negative emotions, but rather as a way to maintain their negative emotional experiences. Based on this model, people with GAD do not necessarily find the experience of a negative emotion distressing; but rather, a shift in their emotional state, from one that is positive or neutral to one that is negative, is experienced as aversive. 
Although the specifics may differ, there are a number of overlapping features across each theory of GAD. In particular, what appears to be common to each theory is that the experience of negative emotions is associated with distress and that worry may be one factor that contributes to the maintenance of distress (Aldao \& Mennin, 2012). Indeed, findings from several laboratory experiments indicate that when people are instructed to worry, they report increased negative affect and decreased positive affect (e.g., Llera \& Newman, 2014; McLaughlin, Borkovec, \& Sibrava, 2007; Stapinski, Abbott, \& Rapee, 2010), which suggests that the very act of worrying may cause distress.

Thus, an aim of the present study is to extend previous laboratory-based examinations of worry by studying the impact of worry on emotional experiences in daily life. By asking people to monitor their current level of worry and intensity of emotions over the course of a day, it is possible to examine the extent to which worry is followed by an immediate change in emotion. This will also shed light on the extent to which worry leads to an increase in negative emotion and decrease in positive emotion, and whether this is more pronounced in people with GAD relative to people with no psychopathology.

\section{Emotional Reactivity to Negative and Positive Events in Daily Life}

Studies of daily life create an opportunity to investigate the impact of daily life events on people's immediate emotional experiences, which cannot be gleaned via cross-sectional or oncea-day diaries. Moreover, examining how self-appraised positive or negative events influence emotional experiences may elucidate patterns of emotional reactivity that are common to, or that differentiate, people with GAD and people with no psychopathology.

A number of ESM studies using nonclinical samples show that daily positive and negative events induce momentary changes in people's emotional experiences. Specifically, 
when people experience a negative event in their daily life, they report a subsequent increase in their negative affect (Gable, Reis, \& Elliot, 2000; Marco, Neale, Schwartz, Shiffman, \& Stone, 1999; van Eck, Nicolson, \& Berkhof, 1998), whereas positive events are related to elevations in positive affect (Langston, 1994). However, positive and negative events may have different effects for people with symptoms of psychopathology. In an ESM study, Peeters, Nicolson, Berkhof, Delespaul and DeVries (2003) reported that people with major depressive disorder displayed blunted negative affect following a negative event in daily life compared to people without depression. In comparison, Suls and Martin (2005) reported that individuals with elevated neuroticism had larger negative affective responses to negative daily events compared to people with low levels of neuroticism.

Although the emotion dysregulation model suggests that people with GAD have heightened reactivity to emotional events, this has yet to be tested in real-life (Fresco et al., 2013; Mennin et al., 2005). Thus, ESM provides an ecologically valid method to clarify the extent to which people with GAD have heightened emotional reactivity to negative or positive life events, relative to people with no psychopathology. In the present study, emotional reactivity is defined as change in the intensity of negative and positive emotion following an external event experienced in daily life (Thompson et al., 2012).

\section{Present Study}

The present study aimed to investigate patterns of emotional experience in people with a diagnosis of GAD compared to people with no psychopathology and to elucidate the dynamic process by which worry and day-to-day negative and positive life events influence momentary changes in people's emotional experiences. As there have been no examinations of everyday emotional experiences with the use of ESM in people with GAD, studies of emotion dynamics of 
major depressive disorder (e.g., Koval, Kuppens, Allen, \& Sheeber, 2012; Moberly \& Watkins, 2008; Thompson et al., 2012), borderline personality disorder (e.g., Trull et al., 2008), and normative samples (e.g., Brans, Koval, Verdyn, Lim, \& Kuppens, 2013) provided a foundation for the study design.

\section{Research Questions and Hypotheses}

\section{Question 1. Do people with GAD exhibit differences in the mean intensity,} instability, and inertia of their daily emotional experiences compared to people with no psychopathology? Based on theory and research, it was expected that within the context of their daily life, people with GAD would report experiencing greater mean intensity of negative emotions relative to people with no psychopathology. The emotion dysregulation model posits that people with GAD have heightened emotional intensity and difficulty with engaging in adaptive emotion regulation strategies to manage their emotional experiences (Fresco et al., 2013; Mennin et al., 2005), it was therefore hypothesized that people with GAD would exhibit greater instability and greater inertia of negative emotions relative to people with no psychopathology. Limited research has examined the intensity of positive emotions in people with GAD relative to people with no psychopathology, however, based on the extant literature on emotion dysregulation in GAD and the literature on emotion dynamics, it was hypothesized that people with GAD would exhibit greater instability, but lower intensity and lower inertia of positive emotions relative to people with no psychopathology.

\section{Question 2. How does worry affect subsequent emotion in people with GAD relative} to people with no psychopathology? By asking people to monitor their current level of worry and intensity of emotions over the course of a day, it is possible to examine the extent to which worry is followed by an immediate change in emotion. Based on the findings from experiments 
that worry is generally associated with increased negative emotion, it was hypothesized that worry would be causally related to an increase in negative emotion, and that this would be significantly more pronounced in people with GAD compared to people with no psychopathology. It was also hypothesized that worry would lead to a significant decrease in positive emotion, however, it was unknown whether this relationship would differ for people with GAD from those with no psychopathology.

\section{Question 3. Do people with GAD experience heightened reactivity to negative and} positive events in their daily life compared to people with no psychopathology? Based on the emotion dysregulation model (Fresco et al., 2013; Mennin et al., 2009), it was hypothesized that people with GAD would report heightened reactivity (i.e., greater increase) in intensity of negative emotions and a greater decrease in intensity of positive emotions following a negative event relative to people with no psychopathology. Given that this was the first known study to examine emotional reactivity to positive events in daily life in people with GAD no a priori hypotheses were proposed. 


\section{Method}

\section{Participants}

The samples consisted of a GAD group and a nonclinical control group (NCC). Participants were recruited from the community via posters and online advertisements, and from the pool of volunteers who had participated in study 1 of this dissertation. All interested individuals were invited to complete a telephone interview to determine eligibility. During the telephone screen, individuals who were 18 years or older were administered the Mini International Neuropsychiatric Interview-7 (MINI 7.0; Sheehan, 2014). Individuals were invited to participate in the study if they endorsed the diagnostic criteria for DSM-5-defined GAD (GAD group), or did not endorse the diagnostic criteria for a current DSM-5-defined psychological disorder (NCC group). In the GAD group, participants were eligible if they reported symptoms that met diagnostic criteria for additional diagnoses, if the symptoms were reported to be less severe than the symptoms associated with GAD.

In addition, people were excluded if they endorsed: current psychotherapy; current or past mania, hypomania, or psychosis; diagnostic criteria for alcohol use disorder or substance use disorder in the past 3 months; and/or clinically significant suicidal ideation, intent, or plan. People taking psychotropic medication were eligible to participate if the dosage was stable for at least 6 weeks. Two individuals who were taking medication met eligibility criteria, however, these individuals did not complete the full study and therefore, their data were not included. A total of 152 participants were screened for the study. Of those screened, 108 individuals met eligibility criteria; however, 19 participants did not start the study and 9 participants were excluded from the data analysis ( $n=2$ equipment error; $n=2$ did not respond to any prompts; $n$ $=5$ restricted range of responses). The final sample comprised of 80 participants; $39 \mathrm{GAD}$ 
participants and 41 NCC participants. Four participants from the GAD group and 5 participants from the NCC group participated in the study 1 of the dissertation.

Participant demographics. Of the 80 participants, $71.3 \%$ were female and $28.7 \%$ were male. Age of participants ranged between 18 and 67 years, with a mean age of 28.26 years $(S D=$ 10.88). The sample was ethnically diverse, with individuals self-identifying as: White (42.5\%), South Asian (20.0\%), East Asian (15.0\%), Black (7.5\%), Mixed (5.0\%), South East Asian (3.7\%), Arab/West Asian (2.5\%), and "Other" (i.e., option not listed) (2.5\%) and Latin American $(1.3 \%)$

The demographic information for the GAD and NCC group is presented in Table 9. The NCC group was significantly older than the GAD group. There were no other significant differences in demographic characteristics between the groups. In the GAD group, individuals with comorbid disorders were included. Comorbid disorders as assessed via the MINI 7.0 included social anxiety disorder (17.5\%), major depressive disorder (13.8\%), panic disorder (7.5\%), obsessive-compulsive disorder (2.5\%) and agoraphobia (1.3\%). Of individuals with comorbid disorders, $18.9 \%$ had one comorbid disorder, $10.0 \%$ had two comorbid disorders and $1.1 \%$ had three comorbid disorders. 
Table 9

Demographic Characteristics Separated by Group

\begin{tabular}{|c|c|c|c|}
\hline & $\begin{array}{c}\text { GAD } \\
n=39\end{array}$ & $\begin{array}{c}\mathrm{NCC} \\
n=41\end{array}$ & $t$ or $\chi^{2}$ \\
\hline Age in years $-\mathrm{M}(\mathrm{SD})^{\mathrm{a}}$ & $25.26(1.06)$ & $31.12(2.07)$ & $2.49 *$ \\
\hline Sex - Frequency $(\%)$ & & & 2.52 \\
\hline Female & $31(79.5)$ & $26(63.4)$ & \\
\hline Male & $8(20.5)$ & $15(36.6)$ & \\
\hline Race/Ethnicity - Frequency (\%) & & & 10.01 \\
\hline Arab/ West Asian & $2(5.1)$ & $0(0.0)$ & \\
\hline Black & $2(5.1)$ & $4(9.8)$ & \\
\hline East Asian & $7(17.9)$ & $5(12.2)$ & \\
\hline Latin American & $1(2.6)$ & $0(0.0)$ & \\
\hline South Asian & $5(12.8)$ & $11(26.8)$ & \\
\hline South East Asian & $2(5.1)$ & $1(2.4)$ & \\
\hline White & $19(48.8)$ & $15(36.6)$ & \\
\hline Mixed & $1(2.6)$ & $3(7.3)$ & \\
\hline Other & $0(0.0)$ & $2(4.9)$ & \\
\hline Marital status- Frequency (\%) & & & 1.25 \\
\hline Single & $33(84.6)$ & $32(78.0)$ & \\
\hline Married/ common-law & $6(15.4)$ & $8(19.5)$ & \\
\hline Divorced/widowed & $0(0.0)$ & $1(2.5)$ & \\
\hline Employment status - Frequency (\%) & & & 1.33 \\
\hline Not working & $18(46.2)$ & $14(34.1)$ & \\
\hline (including student) & & & \\
\hline Working part time & $12(30.8)$ & $14(34.1)$ & \\
\hline Working full time & $9(23.0)$ & $13(31.8)$ & \\
\hline
\end{tabular}

Note. $\mathrm{GAD}=$ generalized anxiety disorder NCC $=$ nonclinical control .

$* p<.05$ 


\section{Materials and Procedure}

Telephone screen. The telephone screen included the administration of the MINI 7.0 (Sheehan, 2014). The MINI is a semistructured diagnostic interview that assesses for DSM-5defined disorders (American Psychiatric Association, 2013). The MINI was administered to assess whether people reported symptoms that met diagnostic criteria for GAD or did not report symptoms that met diagnostic criteria for a DSM 5-defined psychological disorder.

Day 1: Initial laboratory session. Participants attended a 1.5-hour introductory session at the Psychology Research and Training Centre. After providing written informed consent (see Appendix H), participants completed the following self-report measures:

\section{Measures of anxiety and mood symptoms.}

The Penn State Worry Questionnaire (PSWQ; Meyer et al., 1990) is a 16-item self-report measure of worry. Individuals rate each item on a 5-point Likert scale to indicate how characteristic the item is of them $(1=$ not at all typical of me to $5=$ very typical of $m e)$. The PSWQ has demonstrated excellent psychometric properties, including high internal consistency $(\alpha=.86$ to .91 ; Dear et al., 2011) and good test-retest reliability $(r=.74$ to $r=.92$; Startup \& Erickson, 2006). The PSWQ demonstrated excellent internal consistency in the present study ( $\alpha$ $=.96)$.

The State-Trait Inventory for Cognitive and Somatic Anxiety (STICSA; Ree, French, MacLeod, \& Locke, 2008) is made up of two 21-item measures that assess cognitive and somatic anxiety. On the STICSA-Trait people rate how they feel generally, while the STICSA-State people rate how they feel in the moment. Sample items include: "My heart beats fast," and "I think the worst will happen." Items are rated on a 4-point scale $(1=$ Not at all to $4=$ Very much so.). The STICSA-Trait cognitive and somatic subscales have excellent internal consistency (cognitive $\alpha=.95$; somatic $\alpha=.94$ ). The STICSA-State cognitive and somatic subscales also 
have excellent internal consistency (cognitive $\alpha=.94$; somatic $\alpha=.92$; Ree et al, 2008). In the present study, the internal consistency for the trait cognitive and somatic subscales were $\alpha=.92$ and $\alpha=.90$, respectively. The internal consistency for the state cognitive and somatic subscales were $\alpha=.94$ and $\alpha=.90$, respectively.

The Center for Epidemiological Studies-Depression Scale (CES-D; Radloff, 1977) is a 20-item measure of depressive severity. Participants are asked to rate the frequency that they experienced depression symptoms in the past 7 days. Examples of items include "I was bothered by things that don't usually bother me" and "I thought my life has been a failure." Items are rated on a 4 -point scale $(0=$ rarely to $3=$ most or all of the time $)$. The CES-D has good psychometric properties, including good reliability and validity for clinical patients and nonclinical individuals, $\alpha=90$ and $\alpha=.85$, respectively. In addition, the CES-D is found to have good convergent validity with the Beck Depression Inventory (Eaton et al., 2004; Zich et al., 1990). The CES-D demonstrated excellent internal consistency in the present study $(\alpha=.95)$.

\section{Trait measures of emotion and emotion regulation.}

The Affect Intensity Measure (AIM; Larsen \& Deiner, 1987) is a 40-item self-report measure that assesses the frequency with which the individual responds with intense positive and negative reactions to emotional situations. Items are rated on a 6-point scale ranging from 1 (never) to 6 (always). The AIM has demonstrated good internal consistency $(\alpha=.84$; Flett \& Hewitt, 1995) and good test-retest reliability ( $r=0.80$; Larsen \& Deiner, 1987). Scores on the AIM are found to be correlated with people's reported intensity of emotion reactions to negative $(r=.32, \mathrm{p}<.01)$ and positive $(r=.43, \mathrm{p}<.001)$ events in daily life (Larsen et al., 1986). The internal consistency for the AIM was high in the present study $(\alpha=.89)$. 
The Positive and Negative Affect Schedule (PANAS; Watson et al., 1988) is a self-report measure that consists of a 10 -item negative affect scale and a 10-item positive affect scale. The Negative Affect scale assesses a range of aversive affects (e.g., distressed, irritable), whereas the Positive Affect scale assesses a range of pleasant affects (e.g., interested, excited). Respondents rate items on a 5-point scale to assess the extent to which they have experienced that emotion within the past week. The 5-point scale is: 1 (very slightly or not at all), 2 (a little), 3 (moderately), 4 (quite a bit), and 5 (very much so). There is strong support for the internal consistency of the negative and positive subscales, $\alpha=.85$ and .89 , respectively. The PANAS subscales also show strong convergent validity with the Depression Anxiety Stress Scales (Crawford \& Henry, 2004). In the present study, the negative affect scale and the positive affect scale demonstrated strong internal consistency, $\alpha=.93$ and $\alpha=.91$ respectively.

The Emotional Intensity Scale (EIS; Bachorowski \& Braaten, 1994) contains 30 items that assess the intensity of positive and negative emotions without being confounded with the frequency of emotions. Items are rated on a 5-point scale, with lower ratings denoting lesser emotional intensity. The EIS has good internal consistency $(\alpha=.90)$ and good test-retest reliability ( $r=.71$ to .87 ; Bachorowski \& Braaten, 1994). The negative and positive subscales demonstrate good convergence with scores on the AIM (EIS - negative subscale $r=.45, p<.01$; EIS - positive subscale, $r=.48, p<.01)$. The negative subscale shows good convergence with neuroticism subscale of the Eysenck Personality Questionnaire $(r=.64, \mathrm{p}<.01)$, whereas the positive subscale was not associated with neuroticism $(r=.23, \mathrm{p}>.05$; Bachorowski \& Braaten, 1994). The EIS-negative subscale and the EIS - positive subscale demonstrated good internal consistency, $\alpha=.78$ and $\alpha=.91$ respectively. 
The Difficulties in Emotion Regulation Scale (DERS; Gratz \& Roemer, 2004) is a 36item self-report measure of difficulties with emotion regulation, which include difficulties with understanding, accepting, and modifying emotion, a lack of emotional awareness and difficulties engaging in adaptive behaviours during emotional states. Items are rated on a 5-point scale ranging from 1 (not at all) to 5 (completely). Elevated scores on each subscale are indicative of greater dysregulation. Each of the 6 subscales has good psychometric properties, including high internal consistency $(\alpha>.80)$ and good test-retest reliability $(\rho=.88$; Gratz \& Roemer, 2004). The DERS demonstrates good convergent validity with the Generalized Expectancy for Negative Mood Regulation Scale ( $r=-.69, p<.01$; Gratz \& Roemer, 2004). The internal consistency for the DERS total score in the present study was excellent $(\alpha=.96)$.

The Emotion Regulation Questionnaire (ERQ; Gross \& John, 2003) is a 10-item measure of the use of two emotion regulation strategies, expressive suppression (e.g., "I control my emotions by not expressing them") and cognitive reappraisal (e.g., "I control my emotions by changing the way I think about the situation I'm in"). Participants rate their level of agreement from 0 (strongly disagree) to 7 (strongly agree). Gross and John (2003) report that the ERQ has adequate internal consistency ( $\alpha=.79$ for reappraisal and .73 for suppression) and good testretest reliability across 3 months $(r=.69)$. The ERQ has also demonstrated good convergent validity with the negative mood regulation scale (reappraisal subscale: $r=.30, \mathrm{p}<.05$; suppression subscale: $r=-.22, \mathrm{p}<.05)$. In the present study, the cognitive reappraisal subscale and the emotional suppression subscales demonstrated good internal consistency, $\alpha=.86$ and $\alpha=$ .82 respectively.

Experience sampling training. Following completion of the questionnaires, participants were provided with instructions for the use of a smartphone programmed with the movisensXS 
e-diary program (movisens $\mathrm{GmbH}$, Version 0.4 .2437 ), which they used to record their responses. Participants were provided with a smartphone for the duration of the study, or if they had an Android-based smartphone, participants had the option to download the movisensXS application on their own phone ( $40 \%$ used their own phone). Following this training, participants completed a practice trial and their questions were answered. At the end of the initial session, participants were provided with an information sheet and contact information for the experimenter, EJP. All participants were encouraged to contact EJP if any questions arose over the course of the week.

Days 2 to 8: Daily experience sampling. On day 2, all participants started the ESM protocol, which continued for the next 6 consecutive days. Each day, participants were prompted eight times during a 12-hour time period for a total of 56 experience-sampling events (Reis \& Gable, 2000). Participants selected their 12-hour sampling period on Day 1 (e.g., 8am to 8pm; 10 am to $10 \mathrm{pm}$ ). The time required to complete the items at each prompt was brief (approximately 2 minutes) to maximize participant engagement (Nezlek, 2012). Participants were given 15 minutes to respond to each prompt or the opportunity to delay their response if necessary. Participants were contacted during their participation to check if they had any questions about the use of the device or about any of the items.

At each prompt, participants recorded the following in the same order (see Appendix I): Intensity of emotion. Participants rated the intensity of their current experience of 12 distinct emotions on a 9-point scale from 0 (not at all) to 8 (extremely). Negative emotion items included: anxious, angry, sluggish, sad, irritable, and distressed. Positive emotion items included: content, relaxed, enthusiastic, joyful, proud, and interested. For statistical analyses, positive and negative emotions were calculated by averaging the positive and negative items, respectively. These emotion items have been used in previous ESM studies (e.g., Farmer \& Kashdan, 2013; 
Nezlek \& Kuppens, 2008).

Worry. Participants rated the degree to which they engaged in worry since the last prompt from 0 (not at all) to 8 (very much so). Worry was defined as "a type of thought which is concerned with negative future events and is accompanied by anxiety" (Dupuy et al., 2001).

Emotional event. At each prompt, participants were asked if an important emotional event occurred between the current prompt and the previous prompt. If an event occurred, participants were asked to complete the following items (adapted from Bylsma et al., 2011):

1. Identify the event context and if the event included an interaction. Participants selected options from a list provided at each prompt (see Appendix I for the list of options). Participants were also asked to type a brief written statement of what occurred in concrete terms (e.g., argument with friend);

2. Rate how pleasant and unpleasant the event was on a 9 -point scale $(0=$ not at all; $8=$ very). A negative event was coded if the unpleasant rating was equal to or greater than 5 and the pleasant rating was less than 3. A positive event was coded if the pleasant rating was equal to or greater than 5 and the unpleasant rating was less than 3;

3. Rate how stressful the event was on a 9-point scale $(0=$ not at all; $8=$ very $)$; and

4. Rate how important the event was on a 9-point scale $(0=$ not at all; $8=$ very $)$.

Return to laboratory. Following the seventh day of the ESM procedure, participants returned to the laboratory with their smartphone. At this time, the data were downloaded and participants were debriefed about the study hypotheses and implications (see Appendix J). Participants received $\$ 50$ compensation for their participation. 


\section{Statistical Analyses}

Hierarchical linear modeling (HLM), a variant of multiple regression, was used in the present study to analyze the effect of past emotion (from the previous time point), worry, and events on intensity of current negative and positive emotions. HLM is appropriate for use with data sets that have a multilevel structure, such as experience sampling data (see Nezlek, 2012). In the present study, there were two levels of nesting, information provided at each prompt (Level 1), which was nested within each person (Level 2). In addition, HLM is able to account for the high number of missing observations often found in experience sampling studies by using a restricted maximum likelihood approach to estimate the variance-covariance components (Raudenbush \& Bryk, 2002).

In the present study, multilevel models were constructed with separate Level 1 and Level 2 equations using HLM 7.01 software (Raudenbush, Bryk, \& Congdon, 2011). Level 1 equations modeled fluctuations in intensity of emotion. Predictors at Level 1 were person-mean centred (i.e., centred around each person's mean over the 1 week) and the person-level means for the Level 1 predictors were reintroduced at Level 2 (Curran \& Bauer, 2011). Level 2 equations modeled differences in Level 1 predictors as a function of Group, or diagnostic status, which was dummy coded $(\mathrm{NCC}=0, \mathrm{GAD}=1)$. Separate estimates are provided for fixed (significance tests of coefficients) and random (error variances associated with individual effects) effects for each level of the model. 


\section{Results}

\section{Preliminary Analyses}

All study variables were examined for outliers, which were identified as z-score values greater than an absolute value of 3.29 (Tabachnick \& Fidell, 2007). Data points classified as outliers were replaced by the next extreme value. Normality of the data was also assessed by visual inspection and examination of skewness and kurtosis values; it was found that the main study variables approximated a normal distribution.

Clinical characteristics. To determine differences in clinical characteristics between the two groups, a series of independent samples $t$-tests were conducted on scores from the PSWQ, the State and Trait versions of the STICSA, and the CES-D (see Table 10). The GAD group reported significantly greater worry $(d=3.11)$, greater cognitive and somatic symptoms of anxiety at the state $(d=1.43$ and 1.33 , respectively) and trait level ( $d=2.29$ and 1.74 , respectively), and greater depression symptoms $(d=1.69)$ compared to the NCC group.

In the GAD group, $100 \%$ of the sample scored above the cut score of 45 on the PSWQ, which can be used to identify GAD (Behar, Alcaine, Zuellig, \& Borkovec, 2003), and 66.7\% scored above the cut score of 65 on the PSWQ, which can be used to distinguish GAD from another anxiety disorder (Fresco, Mennin, Heimberg, \& Turk, 2003). In the nonclinical control group, only $14.6 \%$ of the sample scored above 45 on the PSWQ and $2.4 \%$ scored above 65 on the PSWQ. The scores in the GAD group are comparable to those with GAD seen in a clinical setting and thus, support the representativeness of the GAD sample. In addition, the scores on the PSWQ in the nonclinical control group are comparable to those observed in other research studies (e.g., Behar et a., 2003; Mennin et al., 2005). 
Table 10

Clinical Characteristics of the GAD and NCC groups

\begin{tabular}{lccccc}
\hline & $\begin{array}{c}\text { GAD } \\
n=39\end{array}$ & $\begin{array}{c}\text { NCC } \\
n=41\end{array}$ & $t$ & $p$ & $d$ \\
\hline PSWQ & $65.67(7.19)$ & $35.12(11.87)$ & $14.00(66.39)$ & $<.001$ & 3.11 \\
STICSA & & & & & \\
State - Cognitive & $22.87(8.20)$ & $13.34(4.64)$ & $6.36(59.44)$ & $<.001$ & 1.43 \\
State - Somatic & $19.31(6.61)$ & $12.54(2.79)$ & $5.92(50.55)$ & $<.001$ & 1.33 \\
Trait - Cognitive & $27.38(6.99)$ & $15.80(4.31)$ & $8.87(62.72)$ & $<.001$ & 2.29 \\
Trait - Somatic & $23.38(6.30)$ & $14.59(3.38)$ & $7.73(57.58)$ & $<.001$ & 1.74 \\
CES-D & $25.87(11.20)$ & $8.62(9.05)$ & $7.60(78)$ & $<.001$ & 1.69 \\
\hline
\end{tabular}

Note. $\mathrm{GAD}=$ generalized anxiety disorder; $\mathrm{NCC}=$ nonclinical control; $\mathrm{PSWQ}=$ Penn State

Worry Questionnaire; STICSA = State-Trait Inventory of Cognitive and Somatic Anxiety; CES-

$\mathrm{D}=$ Center for Epidemiological Studies - Depression. 
Compliance with ESM protocol. The overall compliance rate with the ESM protocol was $78.6 \%$, which indicates that participants responded to, on average, 44 out of the 56 prompts (8 prompts per day for 7 days). This level of compliance was found to be comparable to or better than similar ESM studies (e.g., Bylsma et al., 2011; Thompson et al., 2012). The GAD group ( $M$ $=75.5 \% ; S D=18.7 \%)$ did not differ from the NCC group $(M=80.6 \% ; S D=14.0 \%)$ on their compliance, $t(78)=1.39, p=.17, d=0.31$. In addition, compliance with the protocol was not significantly correlated with age $(r=.18, p=.11)$ or gender $(r=.06, p=.60)$. In terms of clinical characteristics, compliance rates were also not significantly correlated with worry $(r=-$ $.18, p=.11)$, depression severity $(r=-.15, p=.18)$, positive affectivity $(r=.09, p=.42)$ or negative affectivity $(r=-.06, p=.59)$.

\section{Group Differences on Trait Measures of Emotion and Emotion (Dys)regulation}

To examine between group differences on measures of trait emotional intensity, a series of independent samples $t$-tests were performed to compare scores on the AIM, the PANAS, and the negative and positive emotional intensity subscales of the EIS (see Table 11 for means and standard deviations). There were significant differences on the AIM, such that the GAD group reported greater frequency of intense emotions compared to the NCC group $(d=1.02)$. In terms of negative affectivity, the GAD group reported elevated scores on the negative affect subscale of the PANAS $(d=2.17)$ and the negative intensity subscale of the EIS $(d=2.13)$ relative to the NCC group. On the positive emotional intensity measures, compared to the NCC group, the GAD group reported lower positive affect on the PANAS $(d=1.19)$, but did not differ on the positive emotional intensity scale of the EIS $(d=0.19)$.

Next, a series of independent samples $t$-tests were conducted on measures of emotion regulation, including the DERS and the ERQ. On the DERS, the GAD group reported 
significantly greater difficulties with emotion regulation relative to the NCC group $(d=1.83)$. On the ERQ, compared to the NCC group, the GAD group reported significantly lower use of cognitive reappraisal $(d=1.05)$, but did not differ in their use of emotional suppression $(d=$ 0.15). Overall, the differences found between people with GAD and people with no psychopathology on their reported intensity of emotions and difficulties with emotion regulation were consistent with the findings from study 1 in terms of direction and magnitude of the effect. 
Table 11

Means and Standard Deviations of Scores on Emotion-related Questionnaires Separated by Group

\begin{tabular}{|c|c|c|c|c|c|}
\hline & $\begin{array}{c}\text { GAD } \\
n=39\end{array}$ & $\begin{array}{c}\text { NCC } \\
n=41 \\
\end{array}$ & $t$ & $p$ & $d$ \\
\hline \multicolumn{6}{|l|}{ Emotion measures } \\
\hline AIM & $4.01(0.57)$ & $3.49(0.44)$ & $4.52(78)$ & $<.001$ & 1.02 \\
\hline PANAS NA & $30.26(8.07)$ & $15.46(5.27)$ & $9.66(64.91)$ & $<.001$ & 2.17 \\
\hline PANAS PA & $27.56(7.67)$ & $36.44(7.29)$ & $5.31(78)$ & $<.001$ & 1.19 \\
\hline EIS - Negative & $60.36(7.61)$ & $44.07(7.70)$ & $9.51(78)$ & $<.001$ & 2.13 \\
\hline EIS - Positive & $50.38(6.82)$ & $49.22(5.66)$ & $0.83(78)$ & .41 & 0.19 \\
\hline
\end{tabular}

Emotion regulation

$\begin{array}{llllll}\text { DERS } & 101.46(22.07) & 64.95(17.69) & 8.19(78) & <.001 & 1.83\end{array}$

ERQ

$\begin{array}{lllll}\text { Cognitive Reappraisal } \quad 26.77(7.20) & 33.61(5.71) & 4.72(78) & <.001 & 1.05\end{array}$

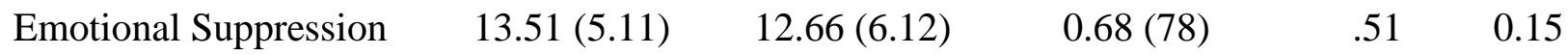

Note. GAD = Generalized Anxiety Disorder; NCC = Nonclinical Control; AIM = Affect Intensity

Measure; PANAS = Positive and Negative Affect Schedule , EIS = Emotion Intensity Scale;

DERS = Difficulties with Emotion Regulation Scale; ERQ = Emotion Regulation Questionnaire. 


\section{Objective 1: Intensity, Instability, and Inertia of Daily Negative and Positive Emotions}

Mean emotion intensity. Two independent samples $t$-tests were performed to determine differences in mean intensity of emotion (negative and positive) between the two groups. For negative emotion, the GAD group reported significantly greater mean negative emotion $(M=$ 2.56, $S D=0.73)$ compared to the NCC group $(M=1.33, S D=1.10), t(78)=5.83, p<.001, d=$ 1.32. For positive emotion, the GAD group reported significantly lower mean positive emotion $(M=3.39, S D=0.98)$ compared to the NCC group $(M=4.58, S D=1.30), t(78)=4.60, p<$ $.001, d=1.03$. The findings indicate that relative to the NCC group, the GAD group reported elevated intensity of negative emotions and lower intensity of positive emotions.

Instability of emotional intensity. Instability is defined as the magnitude of emotional changes from one moment to the next. Mean squared successive differences (MSSD) were calculated as a measure of instability, as MSSD accounts for both the variability and the temporal dependency between two successive emotion intensity ratings. MSSD is a nonmultilevel modeling analysis and is calculated by taking the mean of the squared difference between successive observations across a sampling period divided by the number of observations minus 1 .

$$
M S S D=\frac{1}{N_{\text {observations }}-1} \sum_{\mathrm{i}=1}\left(\text { Emotion }_{t}-\text { Emotion }_{t-1}\right)^{2}
$$

Separate independent samples $t$-tests were then performed to determine differences in MSSD values between the two groups for negative and positive emotions. For negative emotion, the GAD group reported significantly greater instability $(M=2.13, S D=1.78)$ compared to the NCC group $(M=1.04, S D=0.86), t(54.08)=3.48, p=.001, d=0.78$. For positive emotion, the GAD group also reported significantly greater instability $(M=2.19, S D=2.07)$ compared to the 
NCC group $(M=1.36, S D=1.01), t(54.57)=2.26, p=.03, d=0.38$. These findings suggest that the magnitude of the shift in intensity of negative and positive emotional experiences is significantly greater for people in the GAD relative to people in the NCC group.

Emotional inertia. HLM was used to examine emotional inertia (or autocorrelation), which is defined as the extent to which emotion at the previous prompt (i.e., Emotion Em-1 $_{t}$ predicts the emotion at the subsequent prompt $\left(\right.$ Emotion $\left._{t}\right)$. The following analyses for calculating emotional inertia were established by Kuppen and colleagues (2010). To compute the Emotion t- $_{1}$ variable, the first observation of negative emotion for each day was removed and replaced with a missing value, which removes previous day effects (Koval et al., 2012).

The following were of primary interest: (a) the association between Emotion $_{t}($ dependent

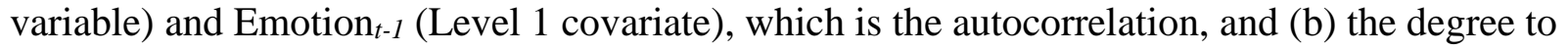
which Group (Level 2 predictor variable) moderated the autocorrelation between Emotion $_{t}$ and Emotion $_{t-1}$. Model testing was conducted in three steps: First, an unconditional model was estimated to determine if there was sufficient between- and within-person variance to meet the conditions of using HLM. Second, a random intercepts model, which includes the Level 1 predictor only, was estimated to determine the degree of autocorrelation in emotion across all participants. Finally, an intercepts-and-slopes-as-outcome model, which includes both Level 1 and Level 2 predictors, was estimated. This final, full model examines the degree to which emotional inertia is moderated by Group status (i.e., GAD or NCC) and indicates the proportion of variance in the intercept and slope that is explained by Group.

An unconditional model was constructed with emotion (negative or positive emotion) as the dependent variable and no Level 1 or Level 2 predictors were included. Separate models were estimated for negative and positive emotion. 
Level 1 Model (within-person: level of prompts):

$$
\text { Emotion }=\beta_{0 j}+r_{i j}
$$

Level 2 Model (between-person: level of participant):

$$
\beta_{0 j}=\gamma_{00}+\mu_{0 j}
$$

In the equation, Emotion ${ }_{\mathrm{ij}}$ represents emotion (negative or positive) for participant $j$ at prompt $i$, and $\mathrm{r}_{i j}$ represents the Level 1 random effect, or the within-person error in predicting Emotion $_{t}$. $\beta_{0 j}$ represents the within-person mean emotion (i.e., the intercept). $\gamma_{00}$ is the grand mean of all person's mean levels of emotion and $\mu_{0 j}$ is the Level 2 (between-persons) random effect.

For negative emotion, the unconditional model revealed significant Level 2 (between person) variance, $\operatorname{var}\left(\mu_{0 j}\right)=1.22, \chi^{2}(79)=4006.54, p<.001$. To determine the proportion of variance in negative emotion that is between-persons, an intraclass correlation coefficient (ICC) was calculated.

$$
I C C=\frac{\tau_{00}}{\tau_{00}+\sigma^{2}}
$$

In this equation, $\tau_{00}$ is the between-person variance and $\sigma^{2}$ is the within-person variance. The ICC was 0.514 , which suggests that $51.4 \%$ of the variance is at the between-persons level and $49.6 \%$ of the variance is at the within-person level. For positive emotion, there was also significant Level 2 variance, $\operatorname{var}\left(\mu_{o j}\right)=1.66, \chi^{2}(79)=5006.10, p<.001$. The intraclass correlation coefficient was 0.567 , indicating that $56.7 \%$ of the variance is at the between-persons level and $43.3 \%$ is at the within-person level. Overall, these findings suggest the need for HLM to account for the clustering of emotion measurements within-person because there is systematic within- and between-persons variance in the dependent variable, Emotion ${ }_{t}$

Next, a random coefficients regression model was examined to determine if there is significant variance in the Level 1 intercept and slope. In this model, Emotion ${ }_{t-1}$ was included as 


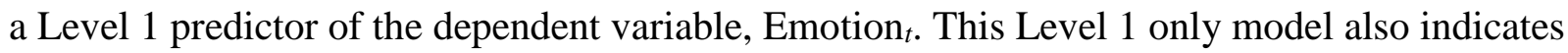
the degree of emotional inertia (or autocorrelation) that was exhibited across all participants.

Level 1 predictors were person-centred (for rationale see, Enders \& Tofighi, 2007; Nezlek, 2012) and the person-level mean emotion $\mathrm{t}_{\mathrm{t}-1}$, which is the aggregate of emotion $\mathrm{w}_{t-1}$ at the prompt level for each participant, was reintroduced at Level 2. No other Level 2 predictors were included in this model. Separate multilevel models were estimated for negative emotion and positive emotion. The equations were as follows:

The Level 1 Model:

$$
\text { Emotion }_{(t) j}=\beta_{0 j}+\beta_{1 j} \text { Person centred Emotion }_{(t-1 j)}+r_{t j}
$$

The Level 2 Model:

$$
\begin{aligned}
& \beta_{0 j}=\gamma_{00}+\mu_{0 j}, \text { and } \\
& \beta_{1 j}=\gamma_{10}+\gamma_{11}\left(\text { Person Level Mean } \text { Emotion }_{(t-1 j)}\right)+\mu_{1 j}
\end{aligned}
$$

In this model, Emotion $(t) j$ represents emotion for person $j$ at prompt $t$ and $\mathrm{r}_{i j}$ represents the Level 1 (within-person) random effect, which is the deviation of each prompt from the predicted value at the prompt and is assumed to be homoscedastic across people. $\beta_{0 j}$ is the within-person mean emotion (negative or positive) and $\beta_{1}$ is the random slope, which represents the degree to which a person's level of emotion at the previous time point $\left(\right.$ Emotion $\left._{t-1}\right)$ predicts their current level of emotion $\left(\right.$ Emotion $_{\mathrm{t}}$ ). The $\gamma_{00}$ and $\gamma_{10}$ represent the intercepts of mean level and autocorrelation of emotion across all participants. The Level 2 (between-persons) random effects, $\mu_{0 j}$ and $\mu_{1 j}$, represent the random effect for the intercept and the random effect of Emotion $_{\mathrm{t}-1}$ after accounting for the effect of the person's mean emotion, respectively.

For negative emotion, $20.3 \%$ of the within-person variance in negative Emotion E $_{t}$ (dependent variable) was accounted for by negative emotion at the prior prompt, Emotion ${ }_{t-1}$ 
(Level 1 predictor). In addition, there was a significant, positive autocorrelation between Emotion $_{t-1}$ and Emotion $t, b=0.77, t(78)=10.34, p<.001$. This finding suggests that across all participants, negative emotions exhibit a degree of inertia, which suggests that intensity of negative emotion at a prior prompt was associated with greater intensity of negative emotion at a subsequent prompt.

For positive emotion, $16.1 \%$ of the within-person variance in positive Emotion $_{t}$ (dependent variable) was accounted for by positive emotion at the prior prompt, Emotion ${ }_{t-1}$ (Level 1 predictor). In addition, there was significant positive emotional inertia across all participants, $b=0.99, t(78)=10.04, p<.001$, which indicates that intensity of positive emotion at a prior prompt is associated with greater intensity of positive emotion at a subsequent prompt.

Finally, to determine the degree to which Group moderated emotional inertia (autocorrelation) of Emotion and $_{t}$ Emotion $_{t-1}$ an intercepts-and-slopes-as-outcomes model was examined. This model builds on the random coefficients model by including Group at Level 2 . Group was dummy-coded $(\mathrm{NCC}=0, \mathrm{GAD}=1)$. Separate models were estimated for negative and positive emotion as the dependent variable.

The Level 1 Model is as follows:

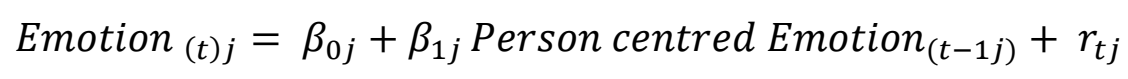

The Level 2 Model consists of the following:

$$
\begin{aligned}
& \beta_{0 j}=\gamma_{00}+\gamma_{01}(\text { Group })+\mu_{0 j}, \text { and } \\
& \beta_{1 j}=\gamma_{10}+\gamma_{11}(\text { Group })+\gamma_{12}(\text { Person Level Mean Emotion }(t-1 j))+\mu_{1 j}
\end{aligned}
$$

In this model, the outcome, Emotion $(t) i j$, represents emotion rating (negative or positive) for participant $j$ at prompt $t$. The outcome is modelled as a function of a random intercept $\beta_{0}$ (i.e., person's mean level of emotion) and a random slope, $\beta_{1}$ (i.e., representing the degree to which a 
person's level of emotion at the previous time point $(t-1)$ predicts their current level of emotion. Due to the dummy coding of Group at Level 2, the intercepts $\gamma_{00}$ and $\gamma_{10}$ represent the mean level and autocorrelation of emotion for people in the NCC group, respectively. The autocorrelation slope is indicative of emotional inertia, as it represents the extent to which a person's current emotion is predicted by their emotion at the prior time point, while controlling for their mean level of emotion (i.e., modeled by the intercept; Koval et al., 2012; Kuppens et al., 2010). At Level 2, the Level 1 intercept and slope were modelled as a function of whether the person was in the NCC group or GAD group. Therefore, slope of $\gamma_{01}$ represents the difference in mean emotion (negative or positive) between the two groups when their lagged Emotion ${ }_{t-1}$ is at the grand mean. In addition, slope $\gamma_{11}$ is the difference in autocorrelation of Emotion ${ }_{t}$ (i.e., effect of lagged Emotion $\mathrm{t}_{\mathrm{t}-1}$, or inertia) between the two groups (Koval et al., 2012). In terms of random effects, $r_{i j}$ is the Level 1 (within-person) random effect, $\mu_{0 j}$ is the Level 2 random effect for the intercept after accounting for the effect of group status, and $\mu_{1 j}$ is the Level 2 random effect of lagged Emotion $_{\mathrm{t}-1}$ after accounting for the effect of group status and the person's mean emotion.

The results are presented in Table 12. For negative emotion, the Level 1 model revealed a significant Emotion $_{t-1}$ slope, which suggests a significant autocorrelation between emotion at time $t$ was predicted by negative emotion at time $t-1$ across all participants. At Level 2, the addition of Group accounted for $30.0 \%$ of the variance in the intercept and $39.0 \%$ of the variance in slope. GAD status was predictive of greater mean negative emotion. In addition, the cross-

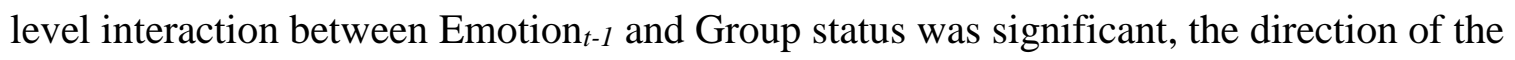
interaction effect indicates that being in the GAD group had a significant, positive effect on the strength of emotional inertia. Thus, people in the GAD group show greater emotional inertia of 
their negative emotion, meaning that they may be more likely to get "stuck" in negative emotions.

For positive emotion, Group accounted for $20.7 \%$ of the variance in the intercept and $3.0 \%$ of the variance in slope, which suggests that group status resulted in a significant difference in mean positive emotion, but did not have a significant role in the degree of autocorrelation of positive emotion. As noted in the results of the previous model, at Level 1, positive emotions displayed significant emotional inertia; people's positive emotion at time $t$ were predicted by their positive emotion at time $t-1$. At Level 2, GAD status was predictive of lower mean positive emotion. The cross-level interaction was significant; the direction of the interaction suggests that the GAD group exhibited lower inertia of positive emotion relative to the control group, indicating that their past emotion is less predictive of the current emotion. 
Table 12

Inertia of Negative and Positive Emotion as a Function of GAD Status

\section{Negative Emotion}

Positive Emotion

\begin{tabular}{|c|c|c|c|c|c|c|c|c|}
\hline Fixed Effect & Coefficient (SE) & $t$ & $d f$ & $p$ & Coefficient (SE) & $t$ & $d f$ & $p$ \\
\hline \multicolumn{9}{|l|}{ For intercept, $\beta_{0}$} \\
\hline Intercept, $\gamma_{00}$ & $1.29(0.15)$ & 8.84 & 78 & $<.001$ & $4.62(0.18)$ & 25.64 & 78 & $<.001$ \\
\hline Group, $\gamma_{01}$ & $1.22(0.21)$ & 5.82 & 78 & $<.001$ & $-1.19(0.26)$ & -4.61 & 78 & $<.001$ \\
\hline \multicolumn{9}{|l|}{ For Emotion $_{t-1}$ slope, $\beta_{1}$} \\
\hline Intercept, $\gamma_{10}$ & $0.52(0.08)$ & 6.87 & 77 & $<.001$ & $1.15(0.12)$ & 9.33 & 77 & $<.001$ \\
\hline Group, $\gamma_{11}$ & $0.38(0.08)$ & 4.67 & 77 & $<.001$ & $-0.18(0.08)$ & -2.20 & 77 & .03 \\
\hline Person-level mean emotion, $\gamma_{12}$ & $-0.22(0.03)$ & -6.50 & 77 & $<.001$ & $-0.19(0.02)$ & -7.79 & 77 & $<.001$ \\
\hline Random effects & $\mathrm{VC}$ & SD & & $p$ & $\mathrm{VC}$ & SD & $p$ & \\
\hline Intercept, $\mu_{0}$ & 0.84 & 0.92 & & $<.001$ & 1.30 & 1.14 & $<.001$ & \\
\hline Slope, $\mu_{1}$ & 0.09 & 0.30 & & $<.001$ & 0.09 & 0.30 & $<.001$ & \\
\hline Level $1, e$ & 0.92 & 0.96 & & & 1.06 & 1.03 & & \\
\hline
\end{tabular}

Note. Level 1 predictors were group-mean centred. Level 2 Group was dummy coded: NCC $=0$, GAD $=1$. Group represents the

contrast between the nonclinical control (NCC) group and the generalized anxiety disorder (GAD) group. VC = Variance component. 
Objective 2: Effect of worry on emotion. An examination of group differences on the effect of worry on intensity of emotion was performed. Level 1 predictors included past worry and emotion t- $_{\text {. Level }} 1$ predictors were person-centered and the person-level means for both predictors were reintroduced at Level 2. At Level 2, group was entered (dummy-coded; NCC = $0, \mathrm{GAD}=1$ ). Separate models were estimated for negative and positive emotion.

Level 1 Model:

$$
\begin{aligned}
& \text { Emotion }_{(t) i j}=\beta_{0 j}+\beta_{1 j} \text { Emotion }_{(t-1)}+ \\
& \beta_{2 j} \text { Person centred Past Worry }+r_{i j}
\end{aligned}
$$

Level 2 Model:

$$
\begin{aligned}
& \beta_{0 j}=\gamma_{00}+\gamma_{01}(\text { Group })+\mu_{0 j}, \text { and } \\
& \beta_{1 j}=\gamma_{10}+\gamma_{11}(\text { Group })+\gamma_{12}(\text { Person level Mean Emotion })+\mu_{1 j}, \text { and } \\
& \beta_{2 j}=\gamma_{20}+\gamma_{21}(\text { Group })+\gamma_{22}(\text { Person level Mean Past Worry })+\mu_{2 j}
\end{aligned}
$$

In this equation, the outcome Emotion $(t) i j$ is the emotion for participant $j$ at prompt $i$ and $r_{i j}$ is the Level 1 (within-person) random effect. $\gamma_{00}$ is the mean emotion for the NCC group, $\gamma_{01}$ is the difference in mean emotion between the two groups and $\mu_{0 j}$ is the Level 2 (between-persons) random effect for the effect of group on Emotion E $\gamma_{11}$ is the difference in slopes between Emotion $_{t}$ and Emotion $t-1$ between the two groups and $\mu_{1 j}$ is the Level 2 random effect of Emotion $_{t-1}$ on Emotion $t$ after accounting for the effect of group and mean level of emotion. Finally, $\gamma_{21}$ is the difference in slopes between Emotion $t$ and Person-level Mean Past Worry between the two groups and $\mu_{2 j}$ is the Level 2 random effect negative emotion after accounting for Group and Person-level Mean Past Worry.

The results are presented in Table 13. At Level 1, there was a positive association between elevated past worry and an increase in negative emotion, as well as, a positive 
autocorrelation of emotion between adjacent prompts. The inclusion of past worry explained an additional $23.3 \%$ of the variance in negative emotion over and above the variance accounted for by the autocorrelation of negative emotion. At Level 2, GAD status predicted a 1.22 increase in mean intensity of negative emotion relative to the control group. There was also a significant cross-level interaction; GAD status led to a stronger association of greater past worry predicting greater negative emotion.

For positive emotion, elevated past worry was associated with a significant decrease in positive emotion, as a well as a positive autocorrelation of emotion between adjacent prompts. The inclusion of past worry explained an additional $9.3 \%$ of the variance in positive emotion over and above the variance accounted for by the autocorrelation of positive emotion from the previous prompt. The inclusion of group status at level 2 was not significant; therefore, no difference was found between the two groups on the impact of worry on subsequent positive emotional intensity. 
Table 13

Emotional Response Following Worry as a Function of GAD Status

\begin{tabular}{|c|c|c|c|c|c|c|c|c|}
\hline \multirow[b]{2}{*}{ Fixed Effect } & \multicolumn{4}{|c|}{ Negative Emotion } & \multicolumn{4}{|c|}{ Positive Emotion } \\
\hline & Coefficient (SE) & $t$ & $d f$ & $p$ & Coefficient (SE) & $t$ & $d f$ & $p$ \\
\hline \multicolumn{9}{|l|}{ For intercept, $\beta_{0}$} \\
\hline Group, $\gamma_{01}$ & $1.22(0.20)$ & 5.96 & 78 & $<.001$ & $-1.20(0.25)$ & -4.72 & 78 & $<.001$ \\
\hline Group, $\gamma_{11}$ & $0.35(0.08)$ & 4.34 & 77 & $<.001$ & $-0.15(0.06)$ & -2.47 & 77 & .02 \\
\hline Person-level mean emotion, $\gamma_{12}$ & $-0.27(0.03)$ & -9.71 & 77 & $<.001$ & $-0.13(0.02)$ & -7.70 & 77 & $<.001$ \\
\hline \multicolumn{9}{|l|}{ For Past Worry slope, $\beta_{2}$} \\
\hline Random Effects & $\mathrm{VC}$ & SD & $p$ & & $\mathrm{VC}$ & SD & $p$ & \\
\hline Intercept, $\mu_{0}$ & 0.85 & 0.92 & $<.001$ & & 1.31 & 1.14 & $<.001$ & \\
\hline Emotion $_{\mathrm{t}-1}$ slope, $\mu_{1}$ & 0.10 & 0.32 & $<.001$ & & 0.05 & 0.22 & $<.001$ & \\
\hline Past worry slope, $\mu_{2}$ & 0.02 & 0.14 & $<.001$ & & 0.01 & 0.11 & $<.001$ & \\
\hline Level $1, e$ & 0.70 & 0.84 & & & 0.98 & 0.96 & & \\
\hline
\end{tabular}

Note. Level 1 predictors were group-mean centred. Level 2 Group was dummy coded: NCC $=0$, GAD $=1$. Group represents the contrast between the nonclinical control (NCC) group and the generalized anxiety disorder (GAD) group. VC = Variance component. 
Effect of emotion on worry. Next, a reverse model was estimated to determine whether changes in negative and positive emotion are causally associated with a subsequent increase in worry. Level 1 predictors included negative emotion ${ }_{t-1}$, positive emotion ${ }_{t-1}$, and worry t $-1 . l^{\text {. Level }} 1$ predictors were person-centered and the person-level means for both predictors were reintroduced at Level 2. At Level 2, Group was entered (dummy-coded; NCC = 0, GAD =1).

Level 1 Model:

$$
\begin{aligned}
& \text { Worry }_{(t) i j}=\beta_{0 j}+\beta_{1 j} \text { Negative Emotion } \\
& (t-1)
\end{aligned}
$$

Level 2 Model:

$$
\begin{aligned}
& \beta_{0 j}=\gamma_{00}+\gamma_{01}(\text { Group })+\mu_{0 j}, \text { and } \\
& \left.\beta_{1 j}=\gamma_{10}+\gamma_{11}(\text { Group })+\gamma_{12} \text { (Person level Mean Negative Emotion }\right) \\
& \quad+\mu_{1 j} \text {, and } \\
& \beta_{2 j}=\gamma_{20}+\gamma_{21}(\text { Group })+\gamma_{22}(\text { Person level Mean Positive Emotion }) \\
& \quad+\mu_{2 j}, \text { and } \\
& \beta_{3 j}=\gamma_{30}+\gamma_{31}(\text { Group })+\gamma_{32}(\text { Person level Mean Worry })+\mu_{3 j}
\end{aligned}
$$

In this equation, the outcome Worry $_{(t) i j}$ is the level of worry for participant $j$ at prompt $i$ and $r_{i j}$ is the Level 1 (within-person) random effect. $\gamma_{00}$ is the mean level of worry for the NCC group, $\gamma_{01}$ is the difference in mean worry between the two groups and $\mu_{0 j}$ is the Level 2 (between-persons) random effect for the effect of group on Worry $t . \gamma_{11}$ is the difference in slopes between Worry $t_{t}$ and Negative Emotion $t_{t-1}$ between the two groups and $\mu_{1 j}$ is the Level 2 random

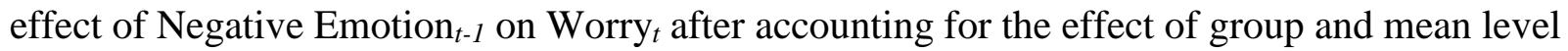
of Emotion. $\gamma_{21}$ is the difference in slopes between Worry and Positive Emotion $_{t-1}$ between the two groups and $\mu_{2 j}$ is the Level 2 random effect of Positive Emotion ${ }_{t-1}$ on Worry $t$ after 
accounting for the effect of group and mean level of Emotion. Finally, $\gamma_{31}$ is the difference in slopes between Worry $t$ and Person-level Mean Worry ${ }_{t-1}$ between the two groups and $\mu_{3 j}$ is the Level 2 random effect negative emotion after accounting for Group and Person-level Mean Worry $_{t-1}$.

The results are presented in Table 14. Neither negative emotion nor positive emotion at the previous time point was associated with greater worry at the subsequent time point. In addition, there was no autocorrelation between current level of worry and level of worry reported at the prior time point for the NCC group, however, there was a significant autocorrelation for the GAD group. The significant autocorrelation indicates that in people with GAD, elevated worry predicted subsequent high worry levels, but this did not appear to be the case for people with no psychopathology.

Taken together, the results of model estimation indicated that increases in worry caused an increase in negative emotion, but that negative emotion did not cause an increase in worry, suggesting a unidirectional relation. Further, worry caused a decrease in positive emotion, but positive emotion did not have a direct effect on subsequent worry. 
Table 14

Relations of Negative and Positive Emotion to Worry as a Function of GAD Status

\begin{tabular}{lcccc}
\hline Fixed Effect & Coefficient (SE) & $t$ & $d f$ & $p$ \\
\hline For intercept, $\beta_{0}$ & & & & \\
$\quad$ Intercept, $\gamma_{00}$ & $1.08(0.20)$ & 5.49 & 78 & $<.001$ \\
$\quad$ Group, $\gamma_{01}$ & $1.68(0.28)$ & 5.96 & 78 & $<.001$ \\
For Negative emotion ${ }_{t-1}$ slope, $\beta_{1}$ & & & & \\
$\quad$ Intercept, $\gamma_{10}$ & $-0.01(0.08)$ & -0.14 & 77 & .89 \\
$\quad$ Group, $\gamma_{11}$ & $-0.001(0.09)$ & 0.01 & 77 & .99 \\
$\quad$ Person-level mean emotion, $\gamma_{12}$ & $0.05(0.04)$ & 1.05 & 77 & .30 \\
For Positive emotion ${ }_{t-1}$ slope, $\beta_{2}$ & & & & \\
$\quad$ Intercept, $\gamma_{20}$ & $0.04(0.14)$ & 0.30 & 77 & .76 \\
$\quad$ Group, $\gamma_{21}$ & $-0.06(0.07)$ & -0.82 & 77 & .42 \\
$\quad$ Person-level mean emotion, $\gamma_{22}$ & $-0.01(0.03)$ & -0.41 & 77 & .68 \\
For Worry $t-1$ slope, $\beta_{3}$ & & & & \\
$\quad$ Intercept, $\gamma_{30}$ & & & & .07 \\
$\quad$ Group, $\gamma_{31}$ & $0.08(0.04)$ & 1.84 & 77 & .02 \\
$\quad$ Person-level mean worry, $\gamma_{32}$ & $0.01(0.02)$ & 0.41 & 77 & .68
\end{tabular}

\begin{tabular}{lccc}
\hline Random Effects & VC & SD & $p$ \\
\hline Intercept, $\mu_{0}$ & 1.54 & 1.24 & $<.001$ \\
Negative emotion ${ }_{t-1}$ slope, $\mu_{1}$ & 0.03 & 0.18 & .37 \\
Positive emotion ${ }_{t-1}$ slope, $\mu_{2}$ & 0.04 & 0.21 & .01 \\
Worry $t-1$ slope, $\mu_{3}$ & 0.02 & 0.16 & $<.001$ \\
Level 1, $e$ & 1.88 & 1.37 & \\
Note. Level 1 predictors were group-mean centred. Level 2 Group was dummy coded: NCC = 0, \\
GAD = 1. Group represents the contrast between the nonclinical control (NCC) group and the \\
generalized anxiety disorder (GAD) group. VC = Variance component.
\end{tabular}


Objective 3: Emotional Reactivity to Negative and Positive Events. Negative and positive events were identified for each participant. The characteristics of the negative and positive events are described in Tables 15, 16, and 17. In terms of differences in types of events between the two groups, the GAD group reported experiencing a greater number of personal failures, "thoughts, ideas or realizations," and fewer goal accomplishments compared to the NCC group. On average, the GAD group reported negative events at a greater frequency, and rated these negative events as more stressful relative to the NCC group. In contrast, the GAD group did not differ in the frequency of positive events compared to the NCC group, but rated positive events as being more stressful. 
Table 15

Percentage of All Reported Event Types Separated by Group

\begin{tabular}{|c|c|c|c|c|c|}
\hline Type of Event & $\begin{array}{c}\text { GAD } \\
n=39 \\
\%(\mathrm{SD})\end{array}$ & $\begin{array}{c}\text { NCC } \\
n=41 \\
\%(\mathrm{SD})\end{array}$ & $t(78)$ & $p$ & $d$ \\
\hline Personal success & $7.49(14.64)$ & $13.07(16.12)$ & 1.62 & .11 & 0.36 \\
\hline Personal Failure & $7.31(8.32)$ & $3.51(5.52)$ & $2.39^{\mathrm{a}}$ & .02 & 0.54 \\
\hline Positive social interaction & $19.41(13.86)$ & $17.05(14.76)$ & 0.74 & .46 & 0.16 \\
\hline Negative social interaction & $15.74(15.59)$ & $10.85(17.68)$ & 1.31 & .19 & 0.29 \\
\hline Neutral social interaction & $5.90(7.42)$ & $4.98(7.08)$ & 0.57 & .57 & 0.13 \\
\hline Thought, idea or realization & $12.56(11.74)$ & $6.17(7.76)$ & 2.89 & .01 & 0.64 \\
\hline Goal accomplished & $5.28(6.97)$ & $9.76(10.17)$ & 2.29 & .03 & 0.51 \\
\hline Goal blocked & $3.44(5.17)$ & $4.78(15.83)$ & 0.51 & .62 & 0.11 \\
\hline $\begin{array}{l}\text { Free from thought/ caught } \\
\text { up in the moment }\end{array}$ & $4.13(6.37)$ & $5.59(8.04)$ & 0.90 & .37 & 0.20 \\
\hline $\begin{array}{l}\text { Reaction to something } \\
\text { heard or seen }\end{array}$ & $6.67(12.47)$ & $8.90(13.07)$ & 0.82 & .44 & 0.18 \\
\hline Other & $12.41(17.56)$ & $12.80(20.58)$ & 0.09 & .93 & 0.02 \\
\hline
\end{tabular}

Note. Frequencies were calculated within individuals, then averaged across individuals. GAD = generalized anxiety disorder; NCC = nonclinical control. Bold indicates a significant difference.

${ }^{\mathrm{a}} \mathrm{df}=65.54$ 
Table 16

Percentage of All Reported Interactions During Events by Group

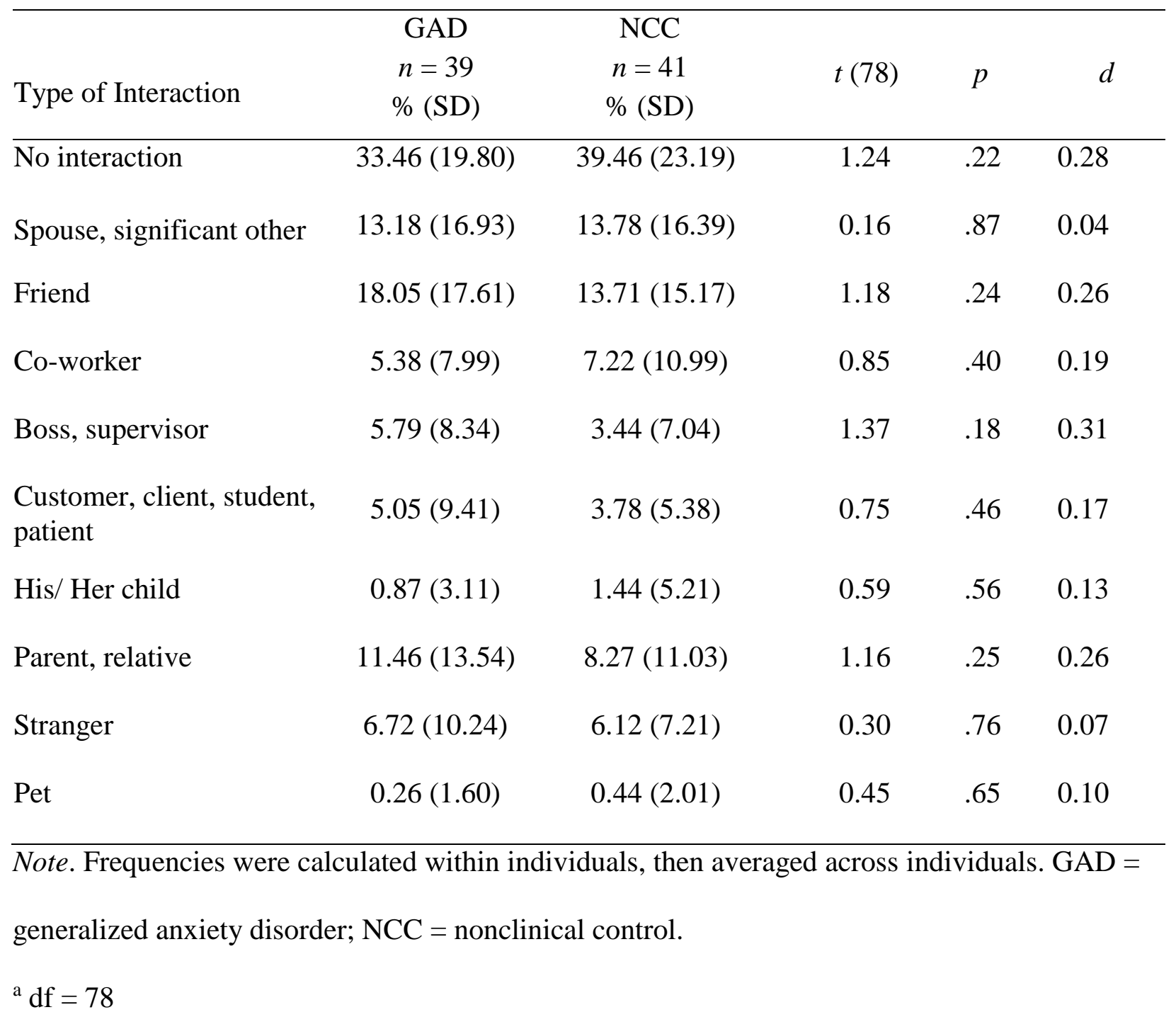


Table 17

Aggregated Mean Ratings for Events Separated by Group

\begin{tabular}{|c|c|c|c|c|c|}
\hline & $\begin{array}{c}\text { GAD } \\
n=39 \\
\mathrm{M}(\mathrm{SD})\end{array}$ & $\begin{array}{c}\mathrm{NCC} \\
n=41 \\
\mathrm{M}(\mathrm{SD})\end{array}$ & $t$ & $p$ & $\bar{d}$ \\
\hline \multicolumn{6}{|l|}{ Negative event } \\
\hline Event frequency & $6.59(4.15)$ & $3.12(2.93)$ & 4.30 & $<.001$ & 0.97 \\
\hline Stressful $^{\mathrm{a}}$ & $5.85(1.54)$ & 4.75 (1.89) & 2.96 & .01 & 0.70 \\
\hline Important $^{\mathrm{a}}$ & $5.04(1.38)$ & $4.24(2.05)$ & 1.92 & .06 & 0.45 \\
\hline \multicolumn{6}{|l|}{ Positive event } \\
\hline Event frequency & $6.67(6.53)$ & $9.10(8.03)$ & 1.48 & .14 & 0.33 \\
\hline Stressful $^{\mathrm{b}}$ & $2.10(1.54)$ & $1.27(1.21)$ & 2.55 & .01 & 0.60 \\
\hline Important $^{\mathrm{b}}$ & $5.38(1.43)$ & $5.37(1.89)$ & 0.03 & .98 & 0.01 \\
\hline
\end{tabular}

Note. Data were calculated within individuals, then averaged across individuals. GAD = generalized anxiety disorder; NCC $=$ nonclinical control. Stressful $=$ Rating of how stressful the event was from 0 (not at all) to 8 (very). Importance $=$ Rating of how important the event was from 0 (not at all) to 8 (very). Bold values indicate significant difference.
${ }^{\mathrm{a}} \mathrm{df}=68$
${ }^{\mathrm{b}} \mathrm{df}=70$ 
To examine differences in emotional reactivity to a negative or positive event in people with GAD compared to healthy controls, four separate models were conducted for reactions to a negative event or a positive event with negative or positive emotion as the outcome variable. At Level 1, Event (positive or negative) was entered as a predictor and was dummy coded $(0=$ no event; 1 = event). At Level 1, person-centered Emotion $\mathrm{t}_{\mathrm{t}-1}$ was entered and the person-level mean emotion was reintroduced at Level 2, to account for the autocorrelation of emotion. At Level 2, Group was entered (dummy-coded; $\mathrm{NCC}=0, \mathrm{GAD}=1$ ).

The Level 1 Model is as follows:

$$
\text { Emotion }_{(t) i j}=\beta_{0 j}+\beta_{1 j} \text { Person centred Emotion }_{(t-1)}+\beta_{2 j} \text { Event }+r_{i j}
$$

The Level 2 Model consists of the following:

$$
\begin{aligned}
& \beta_{0 j}=\gamma_{00}+\gamma_{01}(\text { Group })+\mu_{0 j}, \text { and } \\
& \beta_{1 j}=\gamma_{10}+\gamma_{11}(\text { Group })+\gamma_{12}(\text { Person Level Mean Emotion }(t-1))+\mu_{1 j}, \text { and } \\
& \beta_{2 j}=\gamma_{20}+\gamma_{21}(\text { Group })+\mu_{2 j}
\end{aligned}
$$

In this equation, the outcome Emotion ${ }_{(t) i j}$ is the negative emotion for participant $j$ at prompt $i$ and $r_{i j}$ is the Level 1 (within-person) random effect. $\gamma_{00}$ is the mean negative emotion for the NCC group when no negative event occurred, $\gamma_{01}$ is the difference in mean negative emotion between the two groups when no negative event occurred and $\mu_{0 j}$ is the Level 2 (betweenpersons) random effect for the effect of the intercept after accounting for the effect of Group. $\gamma_{11}$ is the difference in slopes between Emotion $_{t}$ and Emotion Em-l $_{t}$ between the two groups when no event occurred and $\mu_{1 j}$ is the Level 2 random effect of lagged negative emotion $\mathrm{t}_{-1}$ on negative emotion after accounting for the effect of Group and person's Mean Emotion. Finally, $\gamma_{21}$ is the difference between the two groups in the mean difference between an effect occurring and not 
occurring controlling for the effect of Emotion ${ }_{t-1}$ and $\mu_{2 j}$ is the Level 2 random effect of an emotional event on Emotion $_{t}$

Results for negative and positive emotional reactivity to a negative (i.e., unpleasant) event are presented in Table 18. Regarding negative emotional reactivity, at Level 1, both groups reported significant increases in intensity of negative emotion following a negative event. The occurrence of a negative event accounted for $22.6 \%$ of the within-person variance in negative emotion over and above the variance accounted for by the autocorrelation. At Level 2, a significant difference in negative emotional reactivity between the two groups was not found. In terms of the effect of a negative event on positive emotion, at Level 1, both groups reported a significant decrease in positive emotion following a negative event. A negative event occurring accounted for $11.7 \%$ of the within-person variance in positive emotion over and above the variance accounted for by the autocorrelation. Similar to negative emotional reactivity, the two groups were not found to differ in their degree of change in positive emotion following a negative event.

Next, negative and positive emotional reactivity to a positive (i.e., pleasant) event were examined and the findings are presented in Table 19. For negative emotional reactivity, at Level 1 , a positive event resulted in a significant reduction in intensity of negative emotion across the groups. A positive event occurring accounted for $6.1 \%$ of the within-person variance in negative emotion over and above the variance accounted for by the autocorrelation. At Level 2, it was found that the GAD group reported a greater reduction in negative emotion relative to the NCC group following the positive event. For positive emotional reactivity, at Level 1 there was significant increase in positive emotion across groups. A positive event occurring accounted for $12.4 \%$ of the within person variance in positive emotion over and above the variance accounted 
for by the autocorrelation. At Level 2, it was noted that the GAD group reported a significantly greater increase in positive emotion relative to the NCC group following a positive event. 
Table 18

Examining Emotional Reactivity to a Negative Event as a Function of GAD Status

\begin{tabular}{|c|c|c|c|c|c|c|c|c|}
\hline \multirow[b]{2}{*}{ Fixed Effect } & \multicolumn{4}{|c|}{ Negative Emotion } & \multicolumn{4}{|c|}{ Positive Emotion } \\
\hline & Coefficient (SE) & $t$ & $d f$ & $p$ & Coefficient (SE) & $t$ & $d f$ & $p$ \\
\hline \multicolumn{9}{|l|}{ For Intercept, $\beta_{0}$} \\
\hline Intercept, $\gamma_{00}$ & $1.21(0.15)$ & 8.20 & 78 & $<.001$ & $4.69(0.20)$ & 23.44 & 78 & $<.001$ \\
\hline Group, $\gamma_{01}$ & $1.09(0.21)$ & 5.13 & 78 & $<.001$ & $-1.10(0.25)$ & -4.39 & 78 & $<.001$ \\
\hline \multicolumn{9}{|l|}{ For Emotion $_{\mathrm{t}-1}$ slope, $\beta_{1}$} \\
\hline Intercept, $\gamma_{10}$ & $0.72(0.08)$ & 8.48 & 77 & $<.001$ & $0.41(0.09)$ & 4.65 & 77 & $<.001$ \\
\hline Group, $\gamma_{11}$ & $0.57(0.10)$ & 5.42 & 77 & $<.001$ & $0.002(0.05)$ & 0.04 & 77 & .97 \\
\hline Person-level mean emotion, $\gamma_{12}$ & $-0.37(0.03)$ & -13.00 & 77 & $<.001$ & $-0.03(0.02)$ & -1.71 & 77 & .09 \\
\hline \multicolumn{9}{|l|}{ For Negative event slope, $\beta_{2}$} \\
\hline Intercept, $\gamma_{20}$ & $1.09(0.18)$ & 5.85 & 78 & $<.001$ & $-1.15(0.16)$ & -7.26 & 78 & $<.001$ \\
\hline Group, $\gamma_{21}$ & $0.26(0.25)$ & 1.05 & 78 & 0.30 & $0.05(0.19)$ & 0.25 & 78 & 0.81 \\
\hline Random effects & $\mathrm{VC}$ & $\mathrm{SD}$ & 1 & & $\mathrm{VC}$ & $\mathrm{SD}$ & & $p$ \\
\hline Intercept, $\mu_{0}$ & 0.87 & 0.94 & $<.0$ & & 1.28 & 1.13 & & $<.001$ \\
\hline Emotion $_{\mathrm{t}-1}$ slope, $\mu_{1}$ & 0.17 & 0.42 & $<.0$ & & 0.03 & 0.18 & & $<.001$ \\
\hline Negative event slope, $\mu_{2}$ & 0.73 & 0.85 & $<.0$ & & 0.20 & 0.45 & & $<.001$ \\
\hline Level $1, e$ & 0.71 & 0.84 & & & 0.94 & 0.97 & & \\
\hline
\end{tabular}

Note. Group represents the contrast between the nonclinical control (NCC) group and the generalized anxiety disorder (GAD) group.

Dummy codes for group: $\mathrm{NCC}=0, \mathrm{GAD}=1$. $\mathrm{VC}=$ Variance component. 
Table 19

Examining Emotional Reactivity to a Positive Event as a Function of GAD Status

Negative Emotion

Positive Emotion

\begin{tabular}{|c|c|c|c|c|c|c|c|c|}
\hline Fixed Effect & Coefficient (SE) & $t$ & $d f$ & $p$ & Coefficient (SE) & $t$ & $d f$ & $p$ \\
\hline \multicolumn{9}{|l|}{ For Intercept, $\beta_{0}$} \\
\hline Intercept, $\gamma_{00}$ & $1.37(0.18)$ & 7.80 & 78 & $<.001$ & $4.47(0.20)$ & 21.88 & 78 & $<.001$ \\
\hline Group, $\gamma_{01}$ & $1.28(0.21)$ & 6.05 & 78 & $<.001$ & $-1.23(0.25)$ & -4.85 & 78 & $<.001$ \\
\hline \multicolumn{9}{|l|}{ For Emotion $_{\mathrm{t}-1}$ slope, $\beta_{1}$} \\
\hline Intercept, $\gamma_{10}$ & $0.27(0.05)$ & 4.91 & 77 & $<.001$ & $1.21(0.10)$ & 12.48 & 77 & $<.001$ \\
\hline Group, $\gamma_{11}$ & $0.15(0.07)$ & 2.24 & 77 & 0.03 & $-0.21(0.08)$ & -2.66 & 77 & 0.01 \\
\hline Person-level mean emotion, $\gamma_{12}$ & $-0.03(0.03)$ & -1.00 & 77 & 0.32 & $-0.21(0.02)$ & -11.65 & 77 & 0.32 \\
\hline \multicolumn{9}{|l|}{ For Positive event slope, $\beta_{2}$} \\
\hline Intercept, $\gamma_{20}$ & $-0.42(0.08)$ & -5.40 & 78 & $<.001$ & $0.77(0.09)$ & 8.44 & 78 & $<.001$ \\
\hline Group, $\gamma_{21}$ & $-0.50(0.13)$ & -3.85 & 78 & $<.001$ & $0.43(0.15)$ & 2.98 & 78 & .004 \\
\hline Random effects & $\mathrm{VC}$ & SD & $p$ & & $\mathrm{VC}$ & $\mathrm{SD}$ & & $p$ \\
\hline Intercept, $\mu_{0}$ & 0.91 & 0.95 & $<.00$ & & 1.31 & 1.15 & & $<.001$ \\
\hline Emotion $_{\mathrm{t}-1}$ slope, $\mu_{1}$ & 0.03 & 0.19 & $<.00$ & & 0.09 & 0.31 & & $<.001$ \\
\hline Positive event slope, $\mu_{2}$ & 0.12 & 0.35 & $<.00$ & & 0.17 & 0.41 & & $<.001$ \\
\hline Level $1, e$ & 0.85 & 0.92 & & & 0.93 & 0.96 & & \\
\hline
\end{tabular}

Note. Group represents the contrast between the nonclinical control (NCC) group and the generalized anxiety disorder (GAD) group.

Dummy codes for group: $\mathrm{NCC}=0, \mathrm{GAD}=1 . \mathrm{VC}=$ Variance component. 


\section{Discussion}

The present study sought to test tenets of the emotion dysregulation model of GAD, which posits that people with GAD experience emotions at a heightened intensity and engage in maladaptive emotion regulation strategies, including worry, in an attempt to attenuate or control their emotional experiences (Mennin et al., 2005). Specifically, the objectives of the study were to identify the emotional profile of people with GAD and to determine the direct effect that worry and significant events have on their emotional experiences in daily life relative to people with no psychopathology.

\section{Objective 1: Emotional Profile of People with GAD}

The first objective of the study was to clarify the emotional profile exhibited by people with GAD by identifying the characteristics of their negative and positive emotions and to determine how their profile differs from that of people with no psychopathology. The main characteristics of interest were mean intensity, instability, and inertia, as these constructs are argued to be fundamental to understanding the dynamic quality of emotional experiences (Trull et al., 2015).

The hypothesis that people with GAD would experience greater mean intensity, instability, and inertia of negative emotions relative to people with no psychopathology was supported. The findings suggest that in addition to an elevated mean intensity, the negative emotions of people with GAD exhibited greater instability (i.e., change in intensity from one moment to the next) and greater inertia (i.e., autocorrelation of current emotion with prior emotion) relative to those of people with no psychopathology. A graphic representation of this dynamic profile would show large, but slow shifts in negative emotion, which may be likened to being "stuck" in negative emotional states (Koval et al., 2012). 
Considerably less research has examined the intensity, instability, or inertia of positive emotions in daily life. Based on the extant findings, it was hypothesized that people with GAD would exhibit greater instability, but lower mean intensity and lower inertia of positive emotions relative to those of people with no psychopathology. Consistent with the hypothesis, people with GAD reported lower mean intensity of positive emotions, and their emotions exhibited a dynamic pattern reflective of greater instability and lower inertia relative to people with no psychopathology. A graphic representation of this dynamic profile would show large, fast shifts, which would reflect quick fluctuations in positive emotional states.

Taken together, the findings are in agreement with the emotion dysregulation model, which suggests that people with GAD experience negative emotions at a heightened intensity when compared to people with no psychopathology. Alternatively, people with GAD exhibited positive emotions at a lower intensity compared to people with no psychopathology. Beyond identifying general affective experiences of people with GAD, establishing the degree of instability and inertia of their emotions is fundamental to fully understanding their emotional profiles, as both constructs provide a unique perspective of how emotions change over time. The innovative experience sampling design and use of hierarchical linear modeling (HLM) made it possible to study these dynamic constructs.

In the present study, people with GAD exhibited greater instability of negative and positive emotions relative to people with no psychopathology. Greater emotional instability has detrimental effects on emotional well-being, including lower life-satisfaction, elevated neuroticism, and greater symptoms of depression and anxiety; whereas greater stability of emotions is associated with greater psychological well-being (Gruber, Kogan, Quoidbach, \& Mauss, 2013; Houben et al., 2015). One reason that may account for the association between 
instability of emotions and lower psychological well-being is that when people experience instability in their emotions they are more likely to engage in maladaptive emotion regulations strategies (Gross, 2007), which in turn, can be associated with the development of additional stressors (Gratz \& Tull, 2010).

Although emotional instability is often associated with borderline personality disorder (BPD), over the past several years there has been an increase in the amount of research examining emotional instability in mood and anxiety disorders. For example, Trull and colleagues (2008) compared people with BPD to people with depression, and found that although people with BPD showed greater instability on measures of fear, hostility and sadness, they did not differ from people with depression in their instability of positive affect or general negative affect. Moreover, relative to people with no psychopathology, Thompson and colleagues (2012) reported that people with depression reported greater instability of their negative emotions. In terms of anxiety disorders, people with SAD are also found to exhibit greater instability in their negative emotions relative to those of people with no psychopathology (Farmer \& Kashdan, 2013). The presence of unstable positive emotions is less common, however; for example, Farmer and Kashdan (2013) reported that the positive emotions exhibited by people with SAD are found to have greater stability, but lower mean intensity, compared to that of people without SAD. Alternatively, when people with depression are compared to people with no psychopathology, the findings regarding the instability of their positive emotions have been mixed. That is, one study showed that people with depression exhibited greater instability (Koval et al., 2013), whereas another study indicated that the two groups did not differ in their degree of instability (Thompson, 2012). Given that there was no clinical comparison group included in the present study, it is unknown whether the degree of instability exhibited by people with GAD 
would be larger or smaller compared to other clinical groups and therefore, could be a future area of research.

In addition to instability, emotional inertia is argued to be one of the "hallmark [features] of the emotion dynamics that are tied to psychological maladjustment" (Kuppens et al., 2010, p. 985). Greater inertia is indicative of emotions that are "self-predictive" and "self-perpetuating" and therefore, individuals who exhibit greater inertia may be slower to recover from their negative emotional states (Houben et al., 2015). Past findings have shown significant associations between inertia of negative emotions and lower psychological well-being (Houben et al., 2015), greater neuroticism (Kuppens et al., 2010; Suls et al., 1998), elevated depression symptoms (Koval et al., 2012; Koval et al., 2013; Koval et al., 2014; Kuppens et al., 2010; Thompson et al., 2012) and greater rumination (Koval et al., 2012). In addition, greater inertia of negative emotions may be predictive of the development of depression (Kuppens et al., 2012; van de Leemput et al., 2014).

Furthermore, greater inertia may reflect emotions that are resistant to change or an inability to effectively complete regulatory goals (Houben et al., 2015). In particular, Gross (2015) suggests that people may get stuck in emotional experiences for two reasons. They have difficulty taking action towards engaging in an emotion regulation strategy, and therefore, stay on the same emotional course. Alternatively, people may not have the necessary skills to effectively regulate their emotions, even when they are motivated to do so. Indeed, greater emotional inertia has been associated with greater use of ineffective emotion regulation strategies, including emotional suppression (Koval, Butler, Hollenstein, Lanteigne, \& Kuppens, 2015). Therefore, although additional research is needed to understand the factors that are 
associated with greater inertia of negative emotions in people with GAD, one factor may be difficulties with emotion regulation.

Relative to the consequences of elevated inertia of negative emotions, lower inertia of positive emotions may also be detrimental and may reflect ineffective emotion regulation. For example, lower inertia may suggest that people with GAD do not engage in effective strategies to maintain their positive emotions or perhaps that they frequently engage in behaviours, such as worry, which lower their positive emotion. Furthermore, it is possible that people with GAD may actively engage in strategies to diminish their positive emotions. For example, Carl, Fairholme, Gallagher, Thompson-Hollands, and Barlow (2014) conducted an experience sampling study to examine the association between baseline anxiety and depression symptoms and reactions to positive emotions in daily life. The authors reported that greater baseline anxiety and depression symptoms were associated with a greater self-reported effectiveness in ability to minimize positive emotions (i.e., greater agreement with the following statement: After you responded to the event with the behaviors you listed above, to what extent did your positive feelings decrease?). Furthermore, greater baseline anxiety (not depression), was predictive of a greater tendency to endorse the downregulation of positive emotion (i.e., "To what extent did you want to, or were you trying to, decrease your positive feelings?"). The authors interpreted the findings as support that anxiety is associated with deliberate motives to downregulate positive emotions. Taken together, the experience of lower inertia of positive emotions may be due to deliberate efforts to attenuate positive emotions or may be the consequence of specific cognitive and behavioural tendencies. Therefore, the study of cognitive or behavioural antecedents prior to changes in positive emotion would advance this line of research.

Moreover, a future examination of the reasons for why people with GAD exhibit greater 
inertia of negative emotions and lower inertia of positive emotions would be informative and add to the existing theories of GAD. For example, understanding the causes of emotional inertia may provide a test of the contrast avoidance model of worry, which posits that people with GAD may be motivated to maintain a negative emotional state in an effort to avoid an unexpected negative emotional shift (Newman \& Llera, 2011). Thus, a replication and extension of the study by Carl et al. (2014) in people with GAD, as well as other anxiety disorders, may help to clarify whether people with GAD engage in active attempts to maintain their negative emotion and to attenuate their positive emotion.

In summary, the study provides new insights into the dynamic pattern of negative and positive emotions in people with GAD and those with no psychopathology. Prior research has often relied on people's retrospective self-reports of their average emotional intensity or an analysis of the change in emotion between two time points (e.g., difference between pretest and posttest). The experience sampling design provided a novel understanding of the natural ebb and flow of emotions in daily life, including the instability and inertia of emotion. The next step in this line of research is to study the role of external and internal factors, such as thoughts, situational contexts and events, as there is a limited understanding of how these factors influence emotion dynamics. The present study began to address this by providing insight into how worry and events in daily life influence the emotional experiences of those with and without GAD; these findings are discussed in detail in the following sections.

\section{Objective 2: Effect of Worry on Subsequent Emotions}

The second objective of the study was to examine the effect of worry on subsequent emotional experiences. The hypothesis that worry would lead to an increase in negative emotion was supported. Worry resulted in a direct increase in negative emotion, and the association was 
significantly more pronounced in people with GAD relative to people with no psychopathology. Elevated worry also led to a significant decrease in positive emotions, but the degree of association did not differ between the two groups. It is of note that these significant associations were found over and above the variance accounted for by emotional inertia (i.e., the autocorrelation of current emotion with prior emotion), which suggests that the increase in negative emotion following worry was not just driven by prior negative emotion.

This is the first known study to examine the immediate impact of worry on subsequent emotional experiences in daily life. The findings are inconsistent with theoretical models of GAD that suggest that worry may function to inhibit a negative emotional response (Borkovec, 1994; Borkovec et al., 2004; Mennin et al., 2002). Rather, the results are consistent with more recent findings that worry is associated with greater negative emotion (e.g., Llera \& Newman, 2010, 2014; Stapenski, Abbot, \& Rapee, 2010) and are in line with cognitive theories of anxiety, which suggest that worry increases anxiety because it amplifies negative automatic thoughts about the anticipation of threat (e.g., Beck \& Clark, 1997). Furthermore, a recent model of worry, the contrast avoidance model (Newman \& Llera, 2011), posits that worry functions to sustain negative emotion and in turn, to prevent a negative emotional contrast (i.e., a shift from a positive state to an intensely negative state); however, this has not been empirically tested. Thus, research is needed to clarify whether worry is a consciously motivated process, as suggested by the contrast avoidance model, or perhaps better viewed as an unconsciously motivated form of negative automatic thinking, as this understanding would inform interventions to target worry in the treatment of GAD.

In addition, the findings highlight that worry appears to be associated with increased negative emotion for people with and without GAD, but that worry had a significantly worse 
effect on the emotions of those with GAD. Although worry is a universal process and the topics that people with and without GAD report worrying about are similar (Diefenbach, Stanley, \& Beck, 2001; Roemer, Molina, \& Borkovec, 1997), there are several characteristics that distinguish the pathological worry associated with GAD from normative worry. For example, pathological worry is described as chronic, excessive, and uncontrollable (Borkovec, Ray, \& Stöber, 1998; Davey, Tallis, Capuzzo, 1994) and the content is rated as being more abstract, which has detrimental effects on problem solving confidence (Stöber, Tepperwien, \& Staak, 2000). Additionally, pathological worry is related to lower cognitive functioning due to greater utilization of working memory, which may ultimately impair attentional control and lead to the perseveration of worry (Hayes, Hirsch, \& Mathews, 2008). Thus, the pathological quality of worry associated with GAD may account for the greater impact of worry on negative emotion in people with GAD relative to people with no psychopathology.

Overall, a better understanding of the longitudinal relationship between worry and negative affect is needed to understand the maintenance of GAD, and research in other psychological disorders may inform appropriate next steps for the study of this relationship. For example, in the depression literature, Pasyugina, Koval, De Leersnyder, Mesquita, and Kuppens (2015) reported that rumination was associated with a decrease in mood, but did not lead to a direct increase in the severity of depression symptoms. The authors hypothesized rather that rumination may have an indirect role in the maintenance of depression by impairing problem solving abilities and/or tarnishing social supports. Therefore, it is possible that worry maintains negative emotion and the associated symptoms of GAD via indirect pathways, which should be explored in future research. 
Given that worry had a direct effect on subsequent emotion, a second analysis was conducted to examine if the reverse association was also present; that is, whether negative or positive emotion lead to direct changes in their level of worry. Neither negative emotion nor positive emotion was significantly associated with subsequent worry levels. This finding was inconsistent with those of previous studies supporting mood-congruent theories of worry, which suggest that negative mood triggers worry (e.g., Johnston \& Davey, 1997). Alternatively, Davey and Meeten (2016) posit that increased negative mood maintains worry via indirect pathways. Specifically, that a negative mood triggers attentional and interpretive biases that result in the greater detection of threat, and in turn, trigger worry. In addition, they theorized that a negative mood may lead to increased performance standards (i.e., perfectionistic beliefs), which in turn may cause people to perseverate with worry until their worry-related goal (e.g., solving a problem) is perceived to have been met. Thus, a future area of research may be to clarify the extent to which negative mood may function to maintain worry once a person has already started to worry and what, if any, factors may mediate this association.

In addition, the present finding that worry in people with GAD is characterized by greater inertia supports Clark and Beck's (2010) definition of worry as a self-perpetuating cognitive strategy and is consistent with the literature on the perseverative quality of worry in people with this disorder (Davey \& Levy, 1998). To build on the present findings and to better understand the inertia of worry in people with GAD, additional work is needed in the identification of factors that predict the onset and perseveration of worry. For example, based on the extant literature, the activation of worry in GAD may be triggered by perceived threat or loss (Mennin \& Fresco, 2015) or by the goal to avoid experiencing of a negative emotional contrast (Newman \& Llera, 2011). Regarding the perseverative quality of worry, Davey and Meeten (2016) argue that 
additional research is needed to understand the role of negative emotion and maladaptive cognitive processing strategies (including attention and interpretive biases) in maintaining worry. For example, the tendency for people with GAD to catastrophize about their worries (i.e., identify a greater sequence of "what if..." statements; Vasey \& Borkovec, 1992) may have a direct role in the inertia of worry. Taken together, additional research is needed to understand the factors that are associated with the onset and maintenance of worry.

In summary, this is the first known study to examine the direct impact of worry on subsequent emotional experiences in daily life and adds to the existing literature on how worry affects people with GAD differently from those with no psychopathology. Worry and similar perseverative thinking styles are associated with several psychological conditions, including depression (Nolen-Hoeksema, 2000). Studies examining the effect of rumination on emotional experiences report similar findings; such that rumination is associated with a subsequent increase in negative affect and a decrease in positive affect (Pasyugina et al., 2015). Moreover, rumination has also been shown to be associated with a longer duration of negative emotions (Brans \& Verduyn, 2014), which highlights that perseverative negative thinking may cause negative emotions to "stick around" for long periods of time. This is a critical point as individuals who engage in perseverative negative thinking tend to perceive that this form of thinking is helping them in some way (e.g., to gain a sense of certainty, to problem solve, to prepare for a negative outcome; e.g., Borkovec et al., 1999; Dugas, Gagnon, Ladouceur, \& Freeston, 1998), when in actuality, it is just making them feel worse.

\section{Objective 3: Effect of Daily Life Events on Subsequent Emotion}

The third objective was to explore how pleasant or unpleasant events in daily life affect people's emotions. Of the self-identified events in daily life, people with GAD reported a 
significantly greater frequency of unpleasant events, and rated their unpleasant events as being associated with greater levels of stress and greater importance compared to people with no psychopathology. People with GAD also reported a lower frequency of pleasant events compared to people without GAD. Notably, people with GAD rated their pleasant events as being associated with greater stress than people with no psychopathology, but the two groups did not differ in their ratings of how important their positive events were to them. It is possible that positive events were associated with greater stress given the tendency for people with GAD to catastrophize and anticipate that something bad might happen (Hazlett-Stevens \& Craske, 2003).

Of the types of events reported, people with GAD identified experiencing a greater number of personal failures, fewer goals accomplished, and more occasions of being lost in their thoughts, ideas or realizations compared to people with no psychopathology. People with GAD also reported fewer personal successes $(d=0.36)$, however, this was not statistically significant. On the one hand, it is possible that the differences in frequency of events is due to people with GAD experiencing, in general, more unpleasant events in the daily life. On the other hand, because people with GAD have greater negative interpretation biases (e.g., Mathews \& MacLeod, 2005) and high performance standards (Stöber \& Joorman, 2001), they may perceive neutral events as negative events, leading to a greater report of negative events in their daily life. However, given that the responses were subjective, it is not possible to know whether an event that was identified as significantly stressful for one individual would have been appraised in the same manner by a different person, or whether the event really occurred or not. Although this may not be necessary to know, it would be interesting to understand what factors may affect people's perception of events in daily life. 
Regarding the emotional consequences of the events reported, it was hypothesized that people with GAD would report greater emotional reactivity following unpleasant and pleasant events in daily life when compared to individuals with no psychopathology. The hypotheses were partially supported. Following an unpleasant event, both people with GAD and people with no psychopathology, reported experiencing an increase in negative emotion and a decrease in positive emotion, which was over and above the variance accounted for by emotional inertia. Contrary to expectations, however, people with GAD did not react more strongly to unpleasant events in daily life compared to people with no psychopathology. These findings are consistent with the results from the first study, which also showed that people with GAD did not exhibit greater reactivity to a negative emotion induction compared to people with SAD or to people with no psychopathology.

One factor that might account for the lack of differences in emotional reactivity is a person's level of worry prior to the negative event. When people with GAD engage in a fear induction from a worried baseline state, their degree of emotional reactivity is lower than if they started from a neutral or relaxed baseline state (Llera \& Newman, 2014). Therefore, a similar attenuation of emotional reactivity to negative events may occur in daily life if the individual was in a worried state prior to the unpleasant event. The present findings suggest that in daily life, people with GAD are generally in a state of heightened negative emotion and frequently engaging in worry, which may have attenuated their degree of emotional reactivity to events. Thus, a next step in this line of research would be to examine whether level of worry prior to a reported event in daily life is associated with lower emotional reactivity.

The hypotheses regarding emotional reactivity to a pleasant event were supported. Following a pleasant event, both people with GAD and people with no psychopathology reported 
a significant increase in positive emotion and a significant decrease in negative emotion.

Moreover, people with GAD were found to have a greater degree of emotional reactivity, which was accounted for by a significantly greater decrease in negative emotion and greater increase in positive emotion following a pleasant event compared to people with no psychopathology. Again, these findings were over and above the variance accounted for by the autocorrelation of emotion. The finding that people with GAD exhibit heightened reactivity to positive emotions was novel, and inconsistent with the first study, which showed that people with GAD did not report greater reactivity to the positive emotion induction.

Clinical writings highlight the important impact of positive events on emotional wellbeing (Gruber et al., 2013); thus, it is of note that people with GAD responded to pleasant events in daily life by experiencing an increase in their positive emotions and a decrease in their negative emotions. In past research, positive reactivity to pleasant events was found to differentiate people with GAD from those with depression, as depression is associated with reduced emotional reactivity to positive events (Bylsma, Morris, \& Rottenberg, 2008). Moreover, the present findings are consistent with those from a previous laboratory experiment which showed that from a worried, neutral or relaxed state, people with elevated symptoms of GAD respond to a humourous video clip with an increase in happiness and contentment (Llera \& Newman, 2014). Given the benefits of positive emotions to people's psychological well-being, it is a strength that people with GAD respond with increased positive emotion to pleasant events in their daily life. However, additional information is needed on the duration of their positive emotional states. Based on the emotional profile identified for people with GAD, their positive emotions exhibit lower inertia, and therefore, the increase in positive emotion following a positive event may be short-lived. Thus, an empirical examination of the duration of emotional 
experience following an event would provide clarifying information and may suggest that people with GAD require emotion regulation strategies that function to maintain their positive emotions.

\section{Theoretical Implications}

The emotion dysregulation model suggests that people with GAD may be activated and motivated by their steady state of heightened negative emotional arousal to engage in maladaptive emotion regulation strategies to reduce their immediate distress, even when it might lead to long term consequences (Mennin et al., 2002; Mennin et al., 2005). Taken together, the findings provide support that people with GAD experience pervasive negative emotions, which may be affected by a greater tendency to worry and the greater frequency of negative events experienced in their daily life. Moreover, the present findings provide support that worry increases negative affect, and may play a role in keeping people "stuck" in their negative emotions.

Moreover, although the results of the present study are congruent with the contrast avoidance theory, in that people with GAD appear to be in a negative emotional state, in general, and that worry does indeed evoke greater negative emotion in their daily life, it is unknown whether this is due to a deliberate or unconscious desire to avoid an emotional contrast. In addition, the finding that positive emotions were experienced with lower inertia may also reflect a desire to not remain in a positive emotional state for a lengthy period of time. Perhaps this is due to the belief that being in a positive emotional state, one will be at a greater risk or more vulnerable to experiencing a negative emotional contrast, which would be experienced as highly aversive as argued by Newman and Llera (2011); however, this has yet to be tested empirically. Thus, the findings add to the existing literature on emotion in people with GAD and in particular, 
provide greater clarification of their emotional profile, and suggest several new avenues of research.

\section{Strengths, Limitations and Future Directions}

A significant strength of the present study was the use of an innovative research design to test novel research questions about the dynamic characteristics of emotions in the daily life of people with GAD. A benefit of the experience sampling method is the ability to capture emotion, out of the laboratory, as it occurs in daily life (Bolger, Davis, \& Rafaeli, 2003). In addition, with intensive longitudinal data it is possible to examine within-person change, not just betweenperson change (Curran \& Bauer, 2011). The present study also went beyond looking at the general profile of emotions of people with GAD and began to investigate the influence of internal (i.e., worry) and external events on emotion fluctuations, which is the next important step in this line of research (Koval et al., 2013).

In addition to the several strengths of the study, there were also limitations to this study design. First, participants' level of compliance with the ESM protocol varied. However, this did not appear to bias the findings, as the two groups did not differ in their level of compliance with the protocol and none of the study variables were significantly associated with the level of compliance. Second, immediate reactivity was not captured in the present study given that prompts occurred approximately every 1.5 hours; therefore, the emotional response measured in the study was not a "pure" measure of emotional reactivity and may have been compounded by a person's ability to recover following an emotionally evocative event (Koval et al., 2015). Future studies may benefit from using an event-contingent ESM design where participants make a record at the time that a specific event occurs (Reis \& Gable, 2000). Third, a limited number of questions were asked at each prompt to not burden participants. Future studies may examine 
alternative questions, for example, the characteristics of worry, including whether the individual perceived their worry to be uncontrollable, what the person perceived as a trigger of their worry, and use of emotion regulation strategies.

Overall, the findings add to a growing line of research on emotion dynamics in both the fields of affective sciences and clinical psychology. In particular, the study of emotional inertia would benefit from exploring how situational contexts impact emotion dynamics. Kuppens and colleagues (2010) reported that adolescents with depression exhibited higher autocorrelation of negative emotion during highly emotional interpersonal interactions relative to neutral situations. Therefore, future studies may benefit from studying the extent to which a change in emotion or the dynamic nature of an emotion is congruent with a person's current situation, and if incongruent, examining what internal (e.g., perseverative worry; negative interpretation bias) and external factors (e.g., family member arriving home late) may affect the instability and inertia of emotion.

\section{Summary and Conclusion}

The present study provides an initial examination of the dynamic experience of emotions in the daily life of people with GAD, and how their emotions are influenced by their internal and external experiences. Overall, the emotional profile of people with GAD is characterized by negative emotions that have an elevated mean intensity and exhibit greater instability and inertia, whereas their positive emotions have a lower mean intensity and exhibit greater instability, but lower inertia relative to people with no psychopathology. The findings also add to existing theories of worry in GAD, such that worry leads to greater negative emotion, but lower positive emotion, and appears to be self-perpetuating in people with GAD. Finally, the findings highlight that people with GAD experience more self-identified negative events and fewer positive events 
in their daily life, and appear to react more intensely to positive events, but not negative events relative to people with no psychopathology. Taken together, the findings build on the existing literature by providing a more nuanced picture of the emotional profile of people with GAD. 


\section{Chapter 4: Discussion}

Over the past 2 decades, the amount of research on emotion and emotion regulation has grown exponentially (Tull \& Aldao, 2015); however, much remains unknown about the emotional experiences of people with GAD (Mennin et al., 2005). Thus, an aim of the present dissertation was to add to the growing body of research by identifying the emotional profile exhibited by people with GAD. Findings from the two dissertation studies herein highlight that the emotional profile of people with GAD is characterized by greater intensity, instability, and inertia of negative emotions, and lower intensity, greater variability, and lower inertia of positive emotions when compared to that of people with no psychopathology. This type of emotional profile has been associated with greater distress, lower psychological well-being (Houben et al., 2015) and worse physical health (Chalmers, Quintana, Abbott, \& Kemp, 2014) in clinical and nonclinical populations. So, why is this emotional profile maintained? In the following discussion, various motives for self-regulation are explored and related to the extant theories of GAD and worry to highlight the possible motives that may maintain the pattern of heightened negative emotions and lower positive emotions reported by people with GAD. The discussion concludes with a reflection on how understanding their motives for emotion regulation may inform the treatment of GAD.

\section{Motives of Emotion Regulation}

Much of the work on studying emotion and emotion regulation began in the affective sciences literature and has focused on examining how people regulate their emotions (i.e., which emotion regulation strategies people use). Gross (2014) argues that the next step in this line of research is to place greater emphasis on studying why people engage in specific behaviours to upregulate or downregulate emotions. To understand why people engage in specific regulatory 
behaviours requires the identification of their self-regulatory motives or desired outcomes, which may or may not reflect particular emotional end states. Several authors argue that the lack of research focus on motives for emotion regulation is due to a strong theoretical reliance on hedonic principles to explain self-regulatory behaviours (e.g., Higgins, 1997; Martin \& Davies, 1998; Tamir, 2015). The hedonic principle suggests that people are motivated to increase pleasure and decrease pain via the upregulation of positive emotion and downregulation of negative emotion (cf. Gray, 1982; Mees \& Schmitt, 2008). Thus, if people are always driven by hedonic motives, it would not be necessary to look for other possible motives that may drive regulatory behaviours; however, this does not appear to be the case. Indeed, contrary to the hedonic principle, people can also be motivated to experience negative emotions (e.g., Fischer, Manstead, Evers, Timmers, \& Valk, 2004; Hirt \& McCrea, 2000). Therefore, it is necessary to move beyond the reliance on hedonic principles (Higgins, 1997) and to identify other possible motives for why people may engage in behaviours that directly or indirectly modify their emotional experiences.

Two alternative categories of motives have been identified in the affective science literature are contrahedonic motives and instrumental motives (Gross, 2014). Contrahedonic motives are defined as the desire to amplify negative emotions or dampen positive emotions (Riediger, Wrzus, Schmiedek, Wagner, \& Lindenberger, 2011; Tamir, 2015). There are noted benefits of contrahedonic motives, for example, the downregulation of happiness may be appropriate when telling someone bad news (Riediger et al., 2011). However, contrahedonic motives may also be driven by negative beliefs and in turn, encourage people to engage in maladaptive emotion regulatory strategies (e.g., Klonsky, 2007; Riediger et al., 2011). For example, for people with low self-esteem, the belief that they are undeserving of positive moods 
and of positive outcomes is associated with lower engagement in behaviours to upregulate positive emotion (Wood, Heimpel, Manwell \& Whittington, 2009).

Alternatively, instrumental motives are not directly related to an emotional outcome, rather they require people to regulate their emotions for the purpose of reaching a specific nonemotional goal. For example, people may be motivated to endure negative emotions if they hold the belief that it will lead to a positive outcome (e.g., writing an exam to obtain a designation) or activate negative emotions with the aim of improving their odds for success (e.g., increasing anger when negotiating; Tamir, 2015; Tamir, Ford, \& Ryan, 2013). Therefore, in addition to being motivated to modify emotions, there can be other self-regulating factors that encourage people to engage in behaviours that may have a direct or indirect effect on their emotional experiences. Furthermore, individual differences in preferred emotional states may influence the goal of regulatory strategies (Hirt \& McCrea, 2000), as people have different beliefs about their optimal emotional state (Larson, 2000).

\section{Possible Motives for Emotion Regulation in GAD}

What motives might result in emotion (dys)regulation in people with GAD? One of the earliest theories of GAD, Borkovec's avoidance model (e.g., Borkovec, 1994; Borkovec et al., 2004), as well as the emotion dysregulation model (e.g., Mennin et al., 2005) suggest that people with GAD may be driven by hedonic motives. Specifically, the avoidance-based theories argue that people with GAD engage in worry as an emotion regulation strategy with the goal of avoiding or suppressing emotional arousal. However, findings from the present dissertation studies, as well as past research (see Brosschot, Gerin, \& Thayer, 2006; Llera \& Newman, 2010; Stapinski et al., 2010) challenge the notion that worry is associated with the avoidance of emotional arousal. Rather than avoiding or attenuating emotional arousal, these studies report 
that worry leads to an increase in negative emotion. Therefore, additional research is needed to clarify the direction in which people with GAD are motivated to change their emotions. For example, when experiencing negative emotions, people with GAD may be motivated to engage in behaviours to maintain this emotional experience, whereas when they are experiencing positive emotion, they may be motivated to modify this emotional experience.

Moreover, being in a discrepant emotional state can also motivate people to engage in behaviours to return to a perceived sense of homeostasis (Hirt \& McCrea, 2000; Larsen, 2000). Therefore, given that people with GAD have a heightened negative baseline emotional state, they may experience contrahedonic motives to maintain their negative emotional homeostasis. Alternatively, people may be encouraged by the positive beliefs they hold about the experience of negative emotion to maintain a steady negative state. For example, people with GAD report that being in a negative mood state helps them to anticipate and to prepare for future negative events (Borkovec \& Roemer, 1995). Therefore, rather than being motivated to worry with the goal of downregulating negative emotion, people with GAD may have contrahedonic motives to engage in specific emotion regulatory behaviours with the goal of upregulating negative emotion, and one such behaviour may be to worry.

In addition to holding positive beliefs about negative emotions, people with GAD also hold positive beliefs about the utility (e.g., Borkovec, Hazlett-Stevens, \& Diaz, 1999) and consequences (e.g., Davey et al.,1996; Wells, 1999) of worry. Consistent with contrahedonic motives, people with GAD may be motivated to endure the distress associated with worry if they believe it will attenuate the distress associated with negative outcomes in the future. In other words, they prefer to endure distress in the moment in order to not feel worse in the future (Davey et al., 1996). Consistent with this notion, Dugas and Koerner (2005) suggest that worry 
may be an approach-avoidance dilemma; that is, people with GAD are paradoxically motivated to approach a negative emotional state with the purpose of avoiding the experience of an unpredictable negative emotional state. As a result, although worry may be "maladaptive" because it evokes emotional distress, to the individual it may be seen as "adaptive" if it meets his or her regulatory goal (Campbell-Sills, Ellard, \& Barlow, 2014). Moreover, the use of worry as an emotion regulation strategy may be further reinforced by the sense of relief that a person experiences when their feared outcome does not occur (Newman \& Llera, 2011), which may be their ultimate hedonic motive.

The proposal that contrahedonic motives may trigger people with GAD to engage in worry also parallels the contrast avoidance model of worry (Newman \& Llera, 2011). The model posits that people with GAD engage in worry to sustain their negative emotional state as a means to avoid the experience of an unexpected negative emotional shift or emotional contrast, such as a shift from a neutral or positive emotional state to a negative emotional state. Thus, people with GAD may worry in an attempt to avoid an unexpected negative shift in emotion, may be perceived as highly aversive. There is empirical support that engaging in worry prior to a negative experience attenuates people's emotional reactivity (Llera \& Newman, 2014). Taken together, if people are motivated to avoid a negative contrast, and not negative emotions in general, then worry may be perceived as an effective emotion regulation strategy. However, whether people with GAD are motivated to engage in emotion regulatory behaviours to avoid experiencing emotional contrasts has yet to be empirically confirmed.

Although contrahedonic motives are rare when compared to the use of hedonic motives, chronic worry is one example, as people endure the immediate unpleasant emotion that accompanies it with the goal of decreasing the likelihood of experiencing worse pain in the 
future (Tamir, 2015). Despite the fact that people's feared worst case scenarios are often hypothetical and rarely occur, contrahedonic motive to endure negative emotional states may be reinforced and worry may be regarded as an "effective" emotion regulation if they are perceived to have helped them to avoid their feared outcome.

In addition, it is possible that people can hold competing motives at one time. However, instrumental goals are often found to take priority over hedonic motives (Gross, 2014). That is, people may hold hedonic motives, but may also be motivated to experience uncomfortable emotions if they believe that the uncomfortable emotional state will enhance their ability to perform effectively or will lead to a desired outcome (e.g., Tamir, Chiu, \& Gross, 2007; Tamir \& Ford, 2012). For example, Tamir (2005) reported that individuals high in neuroticism perform better on a challenging anagram task when in a worried state (i.e., a mood-congruent state) compared to a neutral state. Moreover, individuals high in neuroticism reported that they preferred to experience worry, despite the associated increase in negative emotion, given their belief that it would increase the likelihood of a future positive outcome. Therefore, it is possible that an instrumental motive, such as "to solve a problem," may activate worry and in turn, increase negative emotion in people with GAD. Taken together, examining people's motives and regulatory goals is necessary as it guides their process of emotion regulation (Gross, 2014; Tamir, 2015).

\section{Treatment of GAD}

GAD is a chronic disorder that is difficult to treat. A meta-analysis found that only $57 \%$ of people with GAD report symptom improvement at 12-months following cognitive therapy to target pathological worry (Hanrahan, Field, Jones \& Davey, 2013). Thus, additional work is needed to improve the therapeutic interventions for people with GAD. As highlighted in the 
current research, people with GAD are found to report a general negative emotional state and thus, assessing the extent to which people are consciously or unconsciously motivated to maintain this emotional profile would be beneficial. Taken together, more information is needed about the specific motives driving emotion regulation in people with GAD and therefore, better incorporation of strategies to assess and target the underlying motives are necessary in the treatment of this disorder.

Although the examination of antecedents and consequences of behaviours via functional analysis has long been a component of behaviour therapy (for a review see Haynes \& O’Brien, 1990), it may need to have a more prominent role in the treatment of GAD. For example, the use of a dialectical behavioural therapy (DBT) behavioural chain analysis may be particularly relevant in the assessment and treatment of GAD as it would help to identify why people with GAD engage in worry as a dysfunctional emotion regulation strategy (Linehan, 1993). In comparison to traditional behavioural analysis, which focuses on broad patterns of behaviour, the chain analysis focuses on specific moment-to-moment changes in people's emotions, thoughts, and behaviors (Linehan, 1993; Lynch, Chapman, Rosenthal, Kuo, \& Linehan, 2006). Therefore, by conducting a chain analysis with an individual with GAD, it would be possible to clearly identify the event that prompts the subsequent chain that cumulates in the engagement of their problematic emotion regulation behaviour, worry.

For example, if people with GAD are motivated to downregulate positive emotions, a prompting event of worry may be "feeling good," which might trigger thoughts about being unprepared for the next bad thing that might happen. In turn, the individual may start to worry in an attempt to gain a sense of control and to downregulate their positive emotion and upregulate their negative emotion. When the negative event that people were worrying about does not occur 
they may experience a sense of relief, which may ultimately reinforce this pattern of behaviour. Thus, conducting a chain analysis would inform what motives may be driving emotion dysregulation and ultimately the maintenance of worry and negative emotions in people with GAD.

Existing therapeutic interventions for people with GAD are not formulated around the idea that people may engage in worry due to contrahedonic motives, but rather some therapies are based on the idea that people with GAD engage in worry as a means of emotional avoidance (e.g., Borkovec et al., 2004; Newman \& Llera, 2011; Roemer \& Orsillo, 2002). In these interventions, emotional avoidance is targeted via exposure techniques (i.e., written and imaginal) to encourage people to think about negative future events and process associated experience of negative emotions and arousal (e.g., Borkovec et al., 2004; Dugas \& Robichaud, 2007; Zinbarg, Craske, \& Barlow, 2006). However, if people with GAD do not actively avoid negative emotion, then asking people to expose themselves to a negative emotional state is unlikely to be effective.

Alternatively, if people with GAD worry as a means to avoid a negative emotional contrast, as suggested by the contrast avoidance model of worry (Newman \& Llera, 2011) then repeated exposure to the distress associated with the experience of a negative emotional contrast (i.e., unexpected shift from a positive or neutral emotional state to a negative emotional state) is necessary for emotional processing to occur in people with GAD. However, contrast exposures have not been tested thus far as a therapeutic intervention. An alternative may be exposure to a positive emotional state, as people with elevated symptoms of GAD endorse having adverse reactions towards positive emotions (e.g., Mennin et al., 2005; Turk et al., 2005). Eagleson, Hayes, Mathews, Perman, and Hirsch (2016) recently conducted a behavioural experiment where 
they had people with GAD engage in positive thinking as a method of reducing worry. For 1 week, participants practiced replacing their worry with positive thoughts or images. At the end of the week, participants reported a significant reduction in their level of worry and anxiety, and these effects were maintained at follow-up 4 weeks later. Thus, engaging in exposure to positive emotions, may challenge their negative beliefs about being in a positive emotional state and subsequently, reduce their level of worry.

Furthermore, given that people with GAD report significant difficulties with emotion dysregulation, including heightened emotional intensity of negative emotions and engagement in ineffective emotion regulation strategies, they may benefit from receiving DBT skills. Neacsiu, Eberle, Kramer, Wiesmann, and Linehan (2014) identified the utility of teaching DBT skills to people with transdiagnostic mood and anxiety disorders as well as underlying difficulties with emotion dysregulation. Compared to receiving an activity-based support group, the authors found that individuals who received DBT skills reported a significant decrease in emotion dysregulation $(d=1.86)$ and decreased anxiety $(d=1.37)$. It is noteworthy that $77.3 \%$ of participants who received DBT skills had a diagnosis of GAD. Thus, there may be benefit to comparing the effectiveness of existing CBT therapies to that of DBT in targeting difficulties with emotion regulation in people with GAD.

Taken together, in combination with psychoeducation about the negative consequences of worry, a target of therapeutic interventions for GAD may be to identify motives for emotion regulation, possibly via a chain analysis. A greater clarification of their motives would provide an understanding of why people with GAD engage in worry despite experiencing an increase in negative emotion and may also elucidate maladaptive beliefs about negative emotional states and the utility of worry. 


\section{Conclusion}

GAD is a chronic disorder characterized by heightened negative emotional intensity and excessive worry. The present dissertation contributes to the current conceptualization of GAD by identifying a dynamic emotional profile that is characterized by greater negative emotion and lower positive emotion. Continued investigation of factors that maintain their emotional profile, including possible motives of emotion regulation, has the potential to add to the emotion dysregulation model and to inform the treatment of GAD. 
Appendices

Appendix A

\section{Mental Imagery Script Task}

[GENERAL TASK INSTRUCTIONS TO READ ALOUD]: In this task, we are interested in learning about two kinds of upcoming events in your life: (1) a happy event and (2) an event that you are worried about occurring. We are interested in having you think about these specific events, which may make you feel extremely happy or extremely worried - and we'd like you to describe this experience in enough detail so a person could understand why this moment will be significant for you.

\section{POSITIVE EVENT: HAPPY EVENT}

To determine a positive event, participants will be asked to think about things that make them the happiest (e.g., accomplishments, successes, or events that were incredibly fulfilling) and think about upcoming events that will make them happy. They will be asked to describe an upcoming event that they consider is one that they are most looking forward to and will make them extremely happy. Once a happy upcoming experience is identified, participants will be asked several questions about specific aspects of the positive scenario to make it more concrete. Specifically, they will be asked to:

(1) Describe what they expect to (1) see, (b) feel, (c) smell, and (d) hear in the situation.

(2) Identify 5 adjectives that describe their anticipated feelings in the situation.

(3) Identify 5 physical sensations that they expect to experience in the situation.

\section{NEGATIVE EVENT: WORST-CASE SCENARIO}

To determine an event that the person is worried about occurring, the experimenter will identify a current worry domain and then use the downward arrow technique to help participants identify a worst-case scenario (see Dugas \& Robichaud, 2007). Once a worst-case scenario is identified, participants will be asked several questions about specific aspects of the worst-case scenario to make it more concrete and to allow them to imagine that the event was happening in the present moment.

Specifically, they will be asked:

(1) Describe what they expect to (1) see, (b) feel, (c) smell, and (d) hear in the situation.

(2) Identify 5 adjectives that describe their anticipated feelings in the situation.

(3) Identify 5 physical sensations that they expect to experience in the situation.

Adapted from Kuo \& Linehan, 2009; Pitman et al., 1987; Yamasaki, 2006 


\section{Positive imagery construction sheet}

Instructions: Please complete the following items with a BRIEF phrase or sentence.

1. Please identify an upcoming situation in which expect that you will feel extremely happy on the following lines:

2. Identify what you see in this situation.

3. Identify sensations of touch in this situation.

4. Identify what you smell in this situation.

5. Identify what you hear in this situation.

\section{Negative imagery construction sheet}

Instructions: Please complete the following items with a BRIEF phrase or sentence.

1. Please identify a situation that is of your greatest current worry of an upcoming event on the following lines:

2. Identify what you see in this situation.

3. Identify sensations of touch in this situation.

4. Identify what you smell in this situation.

5. Identify what you hear in this situation. 


\section{Affect and symptoms checklist}

Select 5 adjectives from the list that describe your feelings in the situation you have identified. Rate the adjectives from $1-5$, with " 1 " being the most prominent feeling and " 5 " being the least prominent.

\begin{tabular}{|c|c|}
\hline interested & irritable \\
\hline distressed & _alert \\
\hline excited & _ashamed \\
\hline _upset & inspired \\
\hline strong & nervous \\
\hline guilty & determined \\
\hline scared & _attentive \\
\hline hostile & jittery \\
\hline enthusiastic & _active \\
\hline proud & _afraid \\
\hline
\end{tabular}

Select 5 symptoms you would be likely to be experiencing in this situation. Rate them from $1-$ 5 , with " 1 " being the most prominent feeling and " 5 " being the least prominent.

Racing heart
Pounding heart
Sweating
Shaking
Short of breath
Choking
Chest pain
Chest discomfort
Nausea
Dizziness

Lightheaded
Feeling as if things around you are unreal
Feeling as if you are detached from yourself
Feeling as if you are losing control
Feeling as if you are going crazy
Feeling as if you are dying
Numb
Tingling
Chills
Hot flashes

Items were taken from the PANAS and ADIS.

Brown, T. A., Barlow, D. H., \& Di Nardo, P. A. (1994). Anxiety Disorders Interview Schedule for DSM-IV (ADIS-IV): Client Interview Schedule. Graywind Publications Incorporated.

Watson, D., Clark, L. A., \& Tellegen, A. (1988). Development and validation of brief measures of positive and negative affect: the PANAS scales. Journal of personality and social psychology, 54, 1063. 


\section{Standard Imagery Script}

You are __ INSERT REFERENCE TO EVENT )_. You notice that you are feeling $\ldots(E M O T I O N \# 5)$ and _ $(E M O T I O N \# 4) \_$. As you look around, you see __(ITEM \#2)_. You smell _(ITEM \#3)_. You feel__(ITEM \#4)_. You hear___(ITEM \#5) Noticing all of the sights, sounds, and smells you are experiencing, you feel _ (EMOTION \#3) _, (EMOTION \#2)_ and _ (EMOTION\#I)_. You notice that _ $($ SENSATION \#5) , _ (SENSATION \#4) _(SENSATION \#3)_. You also notice that you _ (SENSATION \#2)_ and (SENSATION

\#1). For the next several minutes, please continue to imagine, as intensely and as vividly as possible, that you are _ ( INSERT REFERENCE TO EVENT)_. 


\section{Appendix B}

\section{End of Study Follow-up Questions}

During the FIRST listening task:

1. How strongly did you feel you were able to engage with this task?
Disconnected
1

2

3
$\begin{array}{llll}4 & 5 & 6 & 7\end{array}$
8
Very Engaged

2. Were you able to stay focused throughout the task?

Was not able to focus

$\begin{array}{lllllllll}1 & 2 & 3 & 4 & 5 & 6 & 7 & 8 & 9\end{array}$

3. Were you able to complete the task as it was described in the instructions?

\begin{tabular}{llllllllll} 
Not able & & & & & & & & \multicolumn{3}{c}{$\begin{array}{c}\text { Followed all } \\
\text { Instructions }\end{array}$} \\
1 & 2 & 3 & 4 & 5 & 6 & 7 & 8 & 9 & 10
\end{tabular}

4. How difficult was it to follow the instructions for the task?

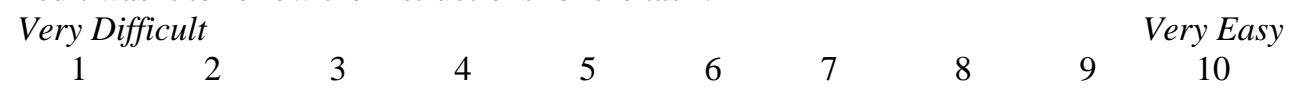

5. Did you do anything to try and distract yourself?

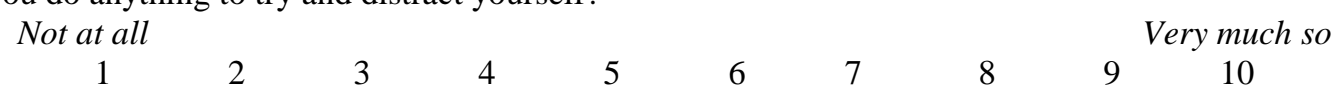

If you tried to distract yourself, what do you do? (please explain in a brief sentence):

During the SECOND listening task:

6. How strongly did you feel you were able to engage with this task?
Disconnected
12
2
$3-4$
$4 \quad 5$
$6 \quad 7$
8
Very Engaged
$9 \quad 10$

7. Were you able to stay focused throughout the task?

\begin{tabular}{ccccccccccc} 
Was not able to focus & & & & & & & \multicolumn{2}{c}{ Very Focused } \\
1 & 2 & 3 & 4 & 5 & 6 & 7 & 8 & 9 & 10
\end{tabular}

8. Were you able to complete the task as it was described in the instructions?

\begin{tabular}{|c|c|c|c|c|c|c|c|}
\hline Not able & & & & & & & $\begin{array}{l}\text { Followed all } \\
\text { Instructions }\end{array}$ \\
\hline $1 \quad 2$ & 3 & 4 & 5 & 6 & 7 & 8 & 10 \\
\hline
\end{tabular}

9. How difficult was it to follow the instructions for the task?
Very Difficult
12
$\begin{array}{lll}3 & 4 & 5\end{array}$
$6 \quad 7$
8
$9 \quad$ Very Easy

10. Did you do anything to try and distract yourself?

$\begin{array}{ccccccccccc}\begin{array}{c}\text { Not at all } \\ 1\end{array} & 2 & 3 & 4 & 5 & 6 & 7 & 8 & 9 & \begin{array}{c}\text { Very much so } \\ 10\end{array}\end{array}$

If you tried to distract yourself, what do you do? (please explain in a brief sentence): 
Appendix $\mathrm{C}$

\section{Experimental Protocol}

1. Imagery Script Development Task

2. Self-report measures

3. Attach physiological sensors

4. Baseline Emotion

- True baseline (5 minutes)

○ Rating of:

i. Subjective emotion

ii. Level of worry

iii. 3) Muscle tension/Bodily arousal

5. Emotion induction 1

- Vanilla baseline (5 minutes)

$\circ$ Rating of

i. Subjective emotion

ii. Level of worry

iii. 3) Muscle tension/Bodily arousal

- Listening to audio recording (approximately 1 minute)

- Imagery (5 minutes)

○ Rating of:

i. Subjective emotion

ii. Level of worry

iii. Muscle tension/Bodily arousal

- Rating of Vividness of mental imagery

- Recovery (5 minutes)

6. Emotion Induction 2

- Vanilla baseline (5 minutes)

○ Rating of

i. Subjective emotion

ii. Level of worry

iii. Muscle tension/Bodily arousal

- Listening to audio recording (approximately 1 minute)

- Imagery (5 minutes)

○ Rating of:

i. Subjective emotion

ii. Level of worry

iii. Muscle tension/Bodily arousal

- Rating of Vividness of mental imagery

- Recovery (5 minutes)

7. Remove physiological sensors

8. Debrief 
Appendix D

\section{Informed Consent Agreement}

Title of Study: Thoughts and Feelings about Upcoming Events Study

You are being asked to participate in a research study. Before you give your consent to be a volunteer, it is important that you read the following information and ask as many questions as necessary to be sure you understand what you will be asked to do.

\section{Investigators:}

Elizabeth Pawluk, MA, Graduate Student, Department of Psychology, Ryerson University

Naomi Koerner, PhD, Associate Professor, Department of Psychology, Ryerson University Martin Antony, PhD, Professor and Chair, Department of Psychology, Ryerson University Janice Kuo, PhD, Assistant Professor, Department of Psychology, Ryerson University

Purpose of the Study: The purpose of this study is to examine how people think and feel about upcoming situations in their lives. The study is being conducted in partial fulfilment of the requirements of Elizabeth Pawluk's PhD in Clinical Psychology at Ryerson University. Drs. Koerner, Antony, and Kuo are supervising the research.

Description of the Study: The study will involve a single visit to the Psychology Research and Training Centre at Ryerson University, located at 105 Bond Street. The total time commitment will be approximately 2 to 2.5 hours.

If you agree to participate, you will be asked to complete the following tasks. (1) A package of questionnaires that contain questions about your thoughts, emotions and behaviour in certain situations.

(2) A task where you will first be asked to describe an upcoming positive event and an upcoming negative event in your life. The details you provide will be inserted into a standard script. Elizabeth Pawluk will then audio record herself reading the script. (3) Finally, you will be played the audio recording of the script and asked to imagine that the events are occurring in the present moment. During the study, we will be monitoring your physiology and will apply a total of 5 electrodes to your skin. Electrodes are small sticky pads that are applied to the skin to monitor your level of perspiration and to measure your heart rate. The electrodes do not hurt to have on, but when you remove them, it may feel the way it does when you remove a Band-Aid. You will be given instructions to place 3 electrodes on your body in private: you will place 2 electrodes on your upper abdomen and 1 electrode below your collarbone. Elizabeth will place one electrode on two fingers of your non-dominant hand (that is, the hand you don't write with).

Potential Risks or Discomforts: There is minimal risk involved in this study. While completing the questionnaires and listening to your scripts you may experience some negative emotions, fatigue or eyestrain. You are able to take breaks as needed throughout the study. You have the right to refuse or discontinue participation at any time. If you decide to stop participating, you will still be entitled to compensation for your time. In addition, you may experience slight discomfort when removing the electrodes, which is similar to removing a Band-Aid.

Potential Benefits of the Study to You or Others: There may be no direct benefits to you for participating in the study. However, you may derive benefit from the self-assessment, as it may increase your awareness of your own emotions and behaviours. You may also develop a better understanding of research methodology and will be providing researchers with valuable insights. 
Confidentiality: Information disclosed in this study will remain completely confidential; however, there are five cases in which the researchers might need to break confidentiality:

(1) if you intend to harm yourself;

(2) if you intend to harm someone else;

(3) if there is reasonable suspicion that a child up to the age of 16 years is at risk of neglect or abuse, we

are required by law to report this to the Children's Aid Society;

(4) if our files are subpoenaed by the courts (records can be opened by a specific court order);

(5) if a regulated health professional has engaged in inappropriate sexual behaviour toward you or another person and you provide us with the name of this individual, we are obligated to report them to their regulatory body.

This informed consent agreement and all written information that you provide will be stored in locked file cabinets at the Psychology Research and Training Centre at Ryerson University. An ID number, as opposed to your name, will be used to identify your study data.

You will be asked to complete questionnaires on a computer using a program called Qualtrics. Your responses will be securely and confidentially stored in a USA-based server. Please note that the data you enter are subject to the Patriot Act, which allows authorities to access the records of internet service providers. If you choose to participate in the study, you understand that your responses to the questions will be stored and accessed in the USA. The security and privacy policy for Qualtrics can be found at the following link: http://www.qualtrics.com/security-statement/.

The audio file generated describing your upcoming events will be encrypted and password protected. These audio files will only be played to you; other participants will not listen to your audio files.

Only people who are directly involved in the study will have access to the information that you provide throughout the study. Your confidentiality will be protected to the full extent allowed by law. Only group findings will be reported in publications and presentations arising from this research. The data will be destroyed 10 years after the findings have been published.

Voluntary Nature of Participation: Participation in this study is completely voluntary. Your choice of whether or not to participate will not influence your future relations with Ryerson University. If you decide to participate, you are free to withdraw your consent and to stop your participation at any time without penalty or loss of benefits to which you are allowed. At any particular point in the study, you may refuse to answer any particular question or stop participation altogether.

Compensation for Participation in the Study: Compensation will be $\$ 25$. You are asked to arrange to transport yourself to the Psychology Research and Training Centre at Ryerson University. You will not be paid for the telephone interview that you completed to determine eligibility.

Questions about the Study: If you have any questions about the research now, please ask. If you have questions about the research later, you may contact Elizabeth Pawluk 416-979-5000 ext. 2182, epawluk@ psych.ryerson.ca, or Dr. Naomi Koerner 416-979-5000 ext. 2151, naomi.koerner@psych.ryerson.ca.

If you have questions regarding your rights as a human participant in this study, you may contact Lynn Lavallée, at the Ryerson University Research Ethics Board for information.

Lynn Lavallée, PhD, Chair of Research Ethics Board

Ryerson University; 350 Victoria St., Toronto, ON

(416)979-5000 ext. 4791; rebchair@ ryerson.ca 
Agreement: Your signature below indicates: (1) that you have read the information in this agreement and have had a chance to ask any questions you have about the Thoughts and Feelings about Upcoming Events Study; (2) that you agree that information collected from you during the telephone interview for the study can be retained and analyzed and (3) that you agree to be in the Thoughts and Feelings about Upcoming Events Study, as described in this consent form and (4) that you have been told that you can change your mind and withdraw your consent to participate at any time. You have been given a copy of this agreement. You have been told that by signing this consent agreement you are not giving up any of your legal rights.

Name of Participant (please print)

Signature of Participant

Signature of Investigator $\quad$ Date

\section{Participation in Future Research}

There is a possibility that members of the Cognition and Psychopathology Laboratory will develop new studies in the future. Please indicate whether or not you agree to be contacted (by email or telephone) to be invited to participate in this future research. Agreeing to be contacted does not obligate you to participate. You are free to turn down any offers to participate in future research, and you are free to withdraw your consent to be contacted at any time.

Yes, I agree to be contacted regarding participation in future research

No, I do not wish to be contacted to participate in future research

If you agree to be contacted for future projects, please provide the best contact method(s):

Email:

Phone: 
Appendix E

\section{Subjective Emotion Rating}

Please rate the extent to which you are currently feeling:

9-point scale from 0 (not at all) to 8 (very much).
1. Anxious
7. Content
2. Relaxed
8. Angry
3. Sluggish
9. Enthusiastic
4. Joyful
10. Sad
5. Irritable
11. Proud
6. Distressed
12. Interested

\section{Current Level of Worry}

On a scale of $0-10$, with " 0 " representing the state of having a blank mind and " 10 " representing the most extreme level of worry you can imagine, how would you rate yourself at this moment? On a scale of $0-10$ :

\section{Current Muscle Tension/Bodily Arousal}

On a scale of $0-10$, with " 0 " representing the state of being absolutely no muscle tension or bodily arousal and " 10 " representing the worst muscle tension or bodily arousal that you can imagine, how would you rate yourself at this moment? On a scale of $0-10$ :

Note. Participants will be prompted to provide these ratings on a computer using a keyboard. 


\section{Appendix F \\ Vividness of Mental Imagery}

Please rate the level of vividness of your mental imagery on a scale of 0 (not at all vivid) to 9 (extremely vivid):

Note. Participants will be prompted to provide these ratings on a computer using a keyboard. 
Appendix G

\section{Thoughts and Feelings about Upcoming Events Study: Consent Form}

Purpose of the Study: Some people report that they experience emotions with greater intensity than do other people they know. In this study, we are interested in examining the extent to which people's emotional responses differ if they are prone to excessive worry, anxiety in social situations, or do not experience excessive worry and anxiety. The way we studied your emotional response in this research was by looking at how you reacted to imagining very vividly something good and something bad happening to you. We measured your heart rate and perspiration throughout our session because research shows that often there is a difference between what a person says he or she is feeling versus what their body shows. So it is very informative to have multiple ways of measuring a person's emotional response to get a more complete picture.

Gaining a better understanding of the emotional response of people who are high (versus low) in worry and anxiety can help inform what type of treatment strategies may be most helpful for those who struggle chronically with worry and anxiety. Research suggests that teaching people how to regulate intense emotions can improve worry and anxiety.

Your willingness to participate in this study is greatly appreciated. Your input will help advance our understanding of ways that difficulties with intense emotions can be alleviated. Our list of resources has titles of books on anxiety and worry management, as well as referral sources (please turn over this page for the list).

Contact Information: If you have any questions or concerns about this experiment or your participation in this study, you may contact:

Elizabeth Pawluk, MA

Main Study Investigator

Department of Psychology

Ryerson University

105 Bond Street

Toronto, ON M5B 2K3

(416) 979-5000 x 2182

caplab@psych.ryerson.ca
Naomi Koerner, $\mathrm{PhD}$

PhD Supervisor

Department of Psychology

Ryerson University

350 Victoria Street

Toronto, ON M5B 2K3

(416) 979-5000 x 2151

naomi.koerner@psych.ryerson.ca
Lynn Lavallée, $\mathrm{PhD}$

Chair, Research Ethics Board

Ryerson University, 350 Victoria Street

Toronto, ON M5B 2K3

(416) 979-5000 x 4791

rebchair@ryerson.ca

If you would like any information about the results of the study once it is complete, please contact Elizabeth Pawluk.

A note about disclosure: In order to maintain the integrity of this research, we ask that you not disclose the purpose of this experiment to others who may be interested in taking part in this study. When participants have too much prior knowledge about the purpose of an experiment, this can affect how they behave in the experiment and the data for that person may not be usable.

\section{Thank you very much for participating in this study!}


Resources: We provide everyone who completes this study with the same list of resources, in case they are interested in learning more about worry or anxiety.

Self-Help Books for Worry and Anxiety

Abramowitz, J.S. (2012). The stress less workbook: Simple strategies to relieve pressure, manage commitments, and minimize conflicts. New York, NY: Guilford Press.

Antony, M.M., \& Norton, P.J. (2009). The anti-anxiety workbook: Proven strategies to overcome worry, panic, phobias, and obsessions. New York, NY: Guilford Press.

Greenberger, D., \& Padesky, C. A. (1995). Mind Over Mood. New York, NY: Guilford Press.

Meares, K., \& Freeston, M. (2008). Overcoming worry: A self-help guide using cognitive behavioral techniques. New York: Basic Books.

Other anxiety resources are available at:

http://www.martinantony.com/links-RecReadingsandVideos.html

\section{Referrals in Toronto Area}

Adult Mental Health Program

Humber River Regional Hospital, Toronto

Contact: Heather Wheeler, Ph.D.

Tel: 416-658-2003

Centre for Addiction and Mental Health

250 College St., Toronto

Tel: 416-979-6819

\section{Private Psychology Referrals}

$\underline{C B T}$ Associates of Toronto

100 Adelaide St. West, Suite 805, Toronto

Tel: 416-363-4228

Web: http://www.cbtassociates.net/

E-Mail: eilenna.denisoff@cbtassociates.net or

peter.farvolden@cbtassociates.net

Hank Frazer, Ph.D., C.Psych.

3852 Finch Ave., Unit 309, Scarborough

Tel: 416-298-9143 or 416-298-1102

Tae Hart, Ph.D., C.Psych.

Tel: 416-473-7132

Email: stacey.hart@psych.ryerson.ca

Trevor Hart, Ph.D., C. Psych

114 Maitland St., Toronto

Tel: 416-979-5000, ext. 1-6192

E-Mail: therapy@drhart.ca
Ryerson University Centre for Student

Development and Counseling

(Available to Ryerson Students Only)

350 Victoria St., Room JOR-07C, Lower

Ground Floor, Jorgenson Hall, Toronto

Tel: 416-979-5195
Randy Katz, Ph.D., C.Psych.

The Clinic

101 DuPont Street, Toronto, ON

Tel: 416-966-1692

Neil Pilkington, Ph.D., C.Psych.

2 Carlton Street, Suite 1718, Toronto

Tel: 416-977-5666

E-Mail: dr.neil.pilkington@ rogers.com

Brian Ridgley, Ph.D.

Ridgley, Thomas, and Associates

60 St. Clair Avenue East, Suite 900, Toronto

Tel: 416-944-3747

E-Mail: brianridgley@ rogers.com

Heather Wheeler, Ph.D., C.Psych.

1333 Sheppard Ave. East, Suite 225, Toronto

Tel: 416-788-3038

E-Mail: hwheeler@rogers.com 
Appendix $\mathrm{H}$

\section{Consent Agreement}

Title of Study: Daily Emotions and Life Experiences Study

You are being asked to participate in a research study. Before you give your consent to be a volunteer, it is important that you read the following information and ask as many questions as necessary to be sure you understand what you will be asked to do.

Investigators: Elizabeth Pawluk, MA, Graduate Student, Department of Psychology, Ryerson University Naomi Koerner, PhD, Associate Professor, Department of Psychology, Ryerson University Martin Antony, PhD, Professor and Chair, Department of Psychology, Ryerson University Janice Kuo, PhD, Assistant Professor, Department of Psychology, Ryerson University

Purpose of the Study: The purpose of this study is to better understand the daily emotional experiences of people who describe worrying a lot and those of people who do not worry very much. The study is being conducted in partial fulfilment of the requirements of Elizabeth Pawluk's PhD in Clinical Psychology at Ryerson University. Drs. Koerner, Antony, and Kuo are supervising the research.

Description of the Study: The study will involve two visits, one-week apart, to the Psychology Research and Training Centre at Ryerson University, located at 105 Bond Street. During the week between visits to the lab, you will be asked to monitor your daily emotional and life experiences multiple times per day on a smartphone (your own, or one borrowed from the lab). The total time commitment will be approximately 4 hours. This study is being funded by the

Canadian Institutes of Health Research. The following is a description of the study:

1. Initial Laboratory Visit (Day 1): You will attend Ryerson University for approximately 1.5 hours. During this visit, you will be asked to complete questionnaires on a computer that contain items about your thoughts, emotions, and behaviour in certain situations. Then you will be provided with instructions for completing the daily monitoring task and complete practice trials of the daily monitoring form on a smartphone. You will receive $\$ 20$ at this visit.

2. Daily Monitoring (Days 2-8): For the next 7 days, following the initial laboratory session, you will be asked to carry either your smartphone or our smartphone with you at all times when completing this portion of the study. Each day, the phone will prompt you 8 times per day (with a ring or a "buzz") over a 12-hour period of your choosing. At each prompt, an application on the smartphone will open and ask you to rate the intensity of the emotions you are experiencing in the moment, report on how much you have been worrying, indicate if a significant emotional event occurred since the previous prompt and answer a few questions about the significant event. You will not be prompted more than once in a 1-hour time span.

3. Return to Laboratory Visit (Day 9): Following completion of monitoring your daily experiences for 7 consecutive days, you will return to the laboratory for approximately half an hour. At this time, you will be asked to return the smartphone (if borrowed) or have the data downloaded from your personal smartphone onto a computer in the lab. You will also be asked to complete questionnaires on a computer. You will receive $\$ 30$ at this visit. 
Potential Risks or Discomforts: There is minimal risk involved in this study. While completing the questionnaires you may experience some negative emotions, fatigue, or eyestrain. You are able to take breaks as needed throughout the study. While completing the daily monitoring, it is possible that you will be prompted to respond at times that are disruptive or inconvenient. To limit the disruption to your daily life, we have made an effort to ensure that the time required at each prompt is minimal (i.e., less than 2 minutes). If necessary, you are able to put the phone on silent for a period of time when you do not wish to be disrupted. It is important for you to know that if you work, go to school, etc. it is likely that you will be prompted to respond during this time. If you believe that this may cause significant problems in your daily life, then this study may not be the best fit for you. In addition, you have the right to refuse or discontinue participation at any time.

Potential Benefits of the Study to You or Others: There may be no direct benefits to you for participating in the study. You may derive benefit from the self-assessment while completing the questionnaires and daily monitoring task, however, as it may increase your awareness of your own emotions and behaviours. By participating, you may also develop a better understanding of research methods and may benefit from the knowledge that you are providing researchers with valuable insights.

Confidentiality: Information disclosed in this study will remain completely confidential; however, there are five cases in which the researchers might need to break confidentiality:

1. if you intend to harm yourself;

2. if you intend to harm someone else;

3. if there is reasonable suspicion that a child up to the age of 16 years is at risk of neglect or abuse, we are required by law to report this to the Children's Aid Society;

4. if our files are subpoenaed by the courts (records can be opened by a specific court order);

5. if a regulated health professional has engaged in inappropriate sexual behaviour toward you or another person and you provide us with the name of this individual, we are obligated to report them to their regulatory body.

This informed consent agreement and all written information that you provide will be stored in locked file cabinets at the Psychology Research and Training Centre at Ryerson University. An ID number, as opposed to your name, will be used to identify your study data. Only people who are directly involved in the study will have access to the information that you provide throughout the study. Your confidentiality will be protected to the full extent allowed by law. Only group findings will be reported in publications and presentations arising from this research. The data will be destroyed 7 years after the final findings have been published.

Questionnaire data. You will be asked to complete questionnaires on a computer using a program called Qualtrics. An ID number will be used to identify your data, which means that your personal identity (e.g., your name) is not known to Qualtrics. Your responses will be securely and confidentially stored in a USA-based server. Please note that the data you enter are subject to the Patriot Act, which allows authorities to access the records of internet service providers. If you choose to participate in the study, you understand that your responses to the questions will be stored and accessed in the USA. The security and privacy policy for Qualtrics can be found at the following link: http://www.qualtrics.com/security-statement 
Daily monitoring data. The responses that you provide on the Smartphone will be entered in a program called MovisensXS and the electronic data will be stored by Host Europe GmbH in Köln, Germany on a secure server. An ID number will be used to identify your data, which means that your personal identity (e.g., your name) is not known to MovisensXS. In addition, to further protect your data, the MovisensXS application uses: (1) Encrypted data storage on the smartphone. When data is encrypted, this means that your information is turned from plain text into an unintelligible format that requires a code to decipher it back to its original form. Therefore, if the smartphone is lost, your information cannot be identified by a third person, because they will not know the code to decipher the information. (2) Encrypted data transfer to the Host Europe GmbH secure server. This means that when your information is sent electronically to the secure server that it is also in an unintelligible format so that people cannot gain access to your information. Host Europe $\mathrm{GmbH}$ is certified for data security and data privacy, and ensures that the server that stores your electronic data is hosted in a secure environment.

Voluntary Nature of Participation: Participation in this study is completely voluntary. Your choice of whether to participate will not influence your relations with Ryerson University. If you decide to participate, you are free to withdraw your consent and to stop your participation at any time without penalty or loss of benefits to which you are allowed. At any point in the study, you may refuse to answer any question or stop participation altogether. If you borrowed a smartphone and decide to stop participating, we ask that you contact the researcher to schedule a time to return the smartphone. If you discontinue participation you are still entitled to compensation for the study phases you began (i.e., \$20 for visit 1 and $\$ 30$ for the daily monitoring phase).

Compensation for Participation in the Study: Compensation will be up to $\$ 50$. You will be compensated $\$ 20$ at the conclusion of the Initial Laboratory Session (day 1) and \$30 when you return to Ryerson University at the end of your participation. You are asked to arrange to transport yourself to the Psychology Research and Training Centre at Ryerson University.

Bonus prize draw - In addition, if you provide valid responses to at least $85 \%$ of the items during the daily monitoring portion of the study, you will be entered into a draw to win a $\$ 100$ VISA gift certificate. Possible reasons for why responses are deemed as invalid are if they are unrealistically quick or if the same answer is provided for all questions. Odds of winning are approximately 1 in 30.

Use of Motorola Moto G Smartphone: If borrowing a smartphone from the laboratory, we ask that you agree to use the smartphone only for the study tasks and that you return the smartphone at the conclusion of your participation. We ask that you please take care of the device. If you fail to return the device, we will contact you at the telephone number provided to arrange for the return of the device. You will not be held financially or legally responsible for the device.

Please initial to indicate that you borrowed a Motorola MOTO G smart phone from the Cognition and Psychopathology Laboratory for the duration of your participation in the Daily Emotions and Life Experiences Study.

Smart Phone ID: 
Questions about the Study: If you have any questions about the research now, please ask. If you have questions about the research later, you may contact Elizabeth Pawluk 416-979-5000 ext. 2188, epawluk@ psych.ryerson.ca, or her research supervisor, Dr. Naomi Koerner 416-979-5000 ext. 2151, naomi.koerner@psych.ryerson.ca.

If you have questions regarding your rights as a human participant in this study, you may contact Lynn Lavallée, at the Ryerson University Research Ethics Board for information. Lynn Lavallée, PhD, Chair of Research Ethics Board Ryerson University; 350 Victoria St., Toronto, ON (416)979-5000 ext. 4791; rebchair@ryerson.ca

Agreement: Your signature below indicates: (1) that you have read the information in this agreement and have had a chance to ask any questions you have about the Daily Emotions and Life Experiences Study; (2) that you agree that information collected from you during the telephone interview for the study can be retained and analyzed; (3) that you agree to be in the Daily Emotions and Life Experiences Study, as described in this consent form and have been told that you can change your mind and withdraw your consent to participate at any time. You have been given a copy of this agreement. You have been told that by signing this consent agreement you are not giving up any of your legal rights.

Name of Participant (please print)

Signature of Participant

Date

Signature of Investigator

Date 
Appendix I

\section{Experience Sampling Method Protocol}

At each prompt, participants will be asked the following questions.

Current Mood ratings, asked at each beep (responses given on a 9-point scale from $0=$ Not at all to $8=$ Extremely):

- How ANXIOUS do you feel right now?

- How ANGRY do you feel right now?

- How SLUGGISH do you feel right now?

- How SAD do you feel right now?

- How IRRITABLE do you feel right now?

- How DISTRESSED do you feel right now?

- How CONTENT do you feel right now?

- How RELAXED do you feel right now?

- How ENTHUSIASTIC do you feel right now?

- How JOYFUL do you feel right now?

- How PROUD do you feel right now?

- How INTERESTED do you feel right now?

Worry ratings, asked at each beep (responses given on a scale from $0=$ not at all to $8=$ very much so)

- How worried have you been since the last prompt? (i.e., thinking about negative future events and feeling anxious).

- How worried do you feel right now?

Emotional event questions, asked at each beep:

- SINCE YOUR LAST REPORT, did an IMPORTANT EMOTIONAL EVENT occur? Yes or no

If a significant emotional event occurred, participants will respond to the following questions:

- What was the NATURE OF THIS EVENT? (select one)

- A personal success

- A personal failure

- A positive social interaction

- A negative social interaction

- A neutral social interaction

- A thought, idea, or realization

- A goal was accomplished

- A goal was blocked

- Being free from thought/caught up in the moment

- A reaction to something I saw or heard

- Other: (please describe)

- Give a brief written statement of what occurred in concrete terms (e.g., argument with friend).

- Were you INTERACTING with someone during the event? 
- If yes, WHO were you interacting with? Check all that apply:

- $\quad$ spouse, significant other

- friends

- co-workers

- boss, supervisor

- clients, customers, students, patients

- my children

- parents, relatives

- strangers

- pets

- Ratings about the event (responses given on a 9-point scale from $0=$ Not at all to $8=$ Extremely):

- How IMPORTANT would you rate this event?

- How PLEASANT would you rate this event?

- How UNPLEASANT would you rate this event?

- How STRESSFUL would you rate this event? 


\section{Appendix $\mathbf{J}$}

\section{Debrief: Daily Emotions and Life Experiences Study}

Purpose of the Study: Some people report that their emotions have greater intensity, more variability, and longer duration than do other people. In this study, we are interested in examining the extent to which people's emotional experiences differ depending on whether they are prone to excessive worry, or do not experience a lot of worry and anxiety. The way we are going to study your day-to-day patterns of emotions is by analyzing your answers to the questions we asked you several times a day over the last week. We are interested in the intensity of your emotional experiences, how your emotional experiences varied over the course of the week, and how long your emotions tended to last. We also are interested in looking at how worrying affected your emotions; it is possible that worry intensifies negative emotions but it is also possible that worrying dampens them to some extent; there is some debate about this in the research literature. The reason we asked you to answer the same questions many times per day, momentto-moment is because this type of monitoring has been shown to capture emotional experiences much more accurately than asking people to reflect back on the last week and remember what emotions they experienced. Gaining a more detailed and accurate understanding of the emotional experiences of people who are high (versus low) in worry and anxiety can help inform what type of treatment strategies may be most helpful for those who struggle with chronic worry and anxiety. Research suggests that teaching people how to regulate intense emotions can improve worry and anxiety.

Your willingness to participate in this study is greatly appreciated. Your input will help advance our understanding of ways that difficulties with intense emotions can be alleviated. Our list of resources has titles of books on anxiety and worry management, as well as referral sources (please turn over this page for the list).

Contact Information: If you have any questions or concerns about this experiment or your participation in this study, you may contact:

Elizabeth Pawluk, MA
Main Study Investigator
Department of Psychology
Ryerson University
105 Bond Street
Toronto, ON M5B 2K3
(416) 979-5000 x 2188
caplab@ psych.ryerson.ca

\author{
Naomi Koerner, $\mathrm{PhD}$ \\ PhD Supervisor \\ Department of Psychology \\ Ryerson University \\ 350 Victoria Street \\ Toronto, ON M5B 2K3 \\ (416) 979-5000 x 2151 \\ naomi.koerner@psych.ryerso \\ n.ca
}

Lynn Lavallée, PhD

Chair, Research Ethics Board

Ryerson University, 350 Victoria Street

Toronto, ON M5B 2K3

(416) 979-5000 x 4791

rebchair@ryerson.ca

If you would like any information about the results of the study once it is complete, please contact Elizabeth Pawluk.

A note about disclosure: In order to maintain the integrity of this research, we ask that you not disclose the purpose of this experiment to others who may be interested in taking part in this study. When participants have too much prior knowledge about the purpose of an experiment, this can affect how they behave in the experiment and the data for that person may not be usable.

Thank you very much for participating in this study!

Resources: We provide everyone who completes this study with the same list of resources, in case they are interested in learning more about worry or anxiety. 
Self-Help Books for Worry and Anxiety

Abramowitz, J.S. (2012). The stress less workbook: Simple strategies to relieve pressure, manage commitments, and minimize conflicts. New York, NY: Guilford Press.

Antony, M.M., \& Norton, P.J. (2009). The anti-anxiety workbook: Proven strategies to overcome worry, panic, phobias, and obsessions. New York, NY: Guilford Press.

Greenberger, D., \& Padesky, C. A. (1995). Mind Over Mood. New York, NY: Guilford Press.

Meares, K., \& Freeston, M. (2008). Overcoming worry: A self-help guide using cognitive behavioral techniques. New York: Basic Books.

Other anxiety resources are available at:

http://www.martinantony.com/links-RecReadingsandVideos.html

Referrals in Toronto Area

OHIP-Covered and Sliding Scale Referrals

Adult Mental Health Program Centre for Addiction and

Humber River Regional

Hospital, Toronto

Contact: Heather Wheeler,

Ph.D.

Tel: 416-658-2003
Mental Health

250 College St., Toronto

Tel: 416-979-6819
Ryerson University Centre for Student Development and Counseling

(Available to Ryerson Students Only)

350 Victoria St., Room JOR-

07C, Lower Ground Floor, Jorgenson Hall, Toronto

Tel: 416-979-5195

Private Psychology Referrals

CBT Associates of Toronto

100 Adelaide St. West, Suite 805, Toronto

Tel: 416-363-4228

Web: http://www.cbtassociates.net/

E-Mail: eilenna.denisoff@cbtassociates.net or peter.farvolden@cbtassociates.net

Hank Frazer, Ph.D., C.Psych.

3852 Finch Ave., Unit 309, Scarborough

Tel: 416-298-9143 or 416-298-1102

Tae Hart, Ph.D., C.Psych.

Tel: 416-473-7132

Email: stacey.hart@psych.ryerson.ca

Trevor Hart, Ph.D., C. Psych

114 Maitland St., Toronto

Tel: 416-979-5000, ext. 1-6192

E-Mail: therapy@drhart.ca
Randy Katz, Ph.D., C.Psych.

The Clinic

101 Dupont Street, Toronto, ON

Tel: 416-966-1692

Neil Pilkington, Ph.D., C.Psych.

2 Carlton Street, Suite 1718, Toronto

Tel: 416-977-5666

E-Mail: dr.neil.pilkington@ rogers.com

Brian Ridgley, Ph.D.

Ridgley, Thomas, and Associates

60 St. Clair Avenue East, Suite 900, Toronto Tel:

416-944-3747

E-Mail: brianridgley@ rogers.com

Heather Wheeler, Ph.D., C.Psych.

1333 Sheppard Ave. East, Suite 225, Toronto

Tel: 416-788-3038

E-Mail: hwheeler@ rogers.com 


\section{References}

Adinoff, B., Mefford, I., Waxman, R., Linnoila, M. (1992). Vagal tone decreases following intravenous diazepam. Psychiatry Research, 41, 89-97. doi: 10.1016/01651781(92)90101-8

Aldao, A., \& Mennin, D. S. (2012). Paradoxical cardiovascular effects of implementing adaptive emotion regulation strategies in generalized anxiety disorder. Behaviour Research and Therapy, 50, 122-130. doi:10.1016/j.brat.2011.12.004

American Psychiatric Association. (2013). Diagnostic and statistical manual of mental disorders ( $5^{\text {th }}$ ed.). Arlington, VA: Author.

Antony, M. M., Coons, M. J., McCabe, R. E., Ashbaugh, A., \& Swinson, R. P. (2006).

Psychometic properties of the Social Phobia Inventory: Further evaluation. Behaviour Research and Therapy, 44, 1177-1185. doi: 10.1016/j.brat.2005.08.013

Bachorowski, J., \& Braaten, E. B. (1994). Emotional intensity: Measurement and theoretical implications. Personality and Individual Differences, 17, 191-199. doi: 10.1016/01918869(94)90025-6

Bagiella, E., Sloan, R. P., \& Heitjan, D. F. (2000). Mixed-effects models in psychophysiology. Psychophysiology, 37, 13-20. doi:10.1111/1469-8986.3710013

Beck, A. T., \& Clark, D. A. (1997). An information processing model of anxiety: Automatic and strategic processes. Behaviour Research and Therapy, 35, 49-58. Doi: 10.1016/S00057967(96)00069-1

Behar, E., Alcaine, O., Zuellig, A. R., \& Borkovec, T. D. (2003). Screening for generalized anxiety disorder using the Penn State Worry Questionnaire: A receiver operating characteristic analysis. Journal of Behavior Therapy and Experimental Psychiatry, 34, 
25-43. doi: 10.1016/S0005-7916(03)00004-1

Behar, E., DiMarco, I. D., Hekler, E. B., Mohlman, J., \& Staples, A. M. (2009). Current theoretical models of generalized anxiety disorder (GAD): Conceptual review and treatment implications. Journal of Anxiety Disorders, 23, 1011-1023. doi:

10.1016/j.janxdis.2009.07.006

Berntson, G. G., Quigley, K. S., \& Lozano, D. (2007). Cardiovascular psychophysiology. In J. T. Cacioppo, L. G. Tassinary, G. G. Louis (Eds.), Handbook of psychophysiology (3rd ed., pp. 182-210). New York, NY: Cambridge University Press.

Bolger, N., Davis, A., \& Rafaeli, E. (2003). Diary methods: Capturing life as it is lived. Annual Review of Psychology, 54, 579-616. doi:10.1146/annurev.psych.54.101601.145030

Borkovec, T. D. (1994). The nature, functions and origins of worry. In G. C. L. Davey \& F. Tallis (Eds.), Worrying: Perspectives on theory, assessment, and treatment (pp. 5-33). Oxford, UK: John Wiley \& Sons.

Borkovec, T. D., Alcaine, O. M., \& Behar. E. (2004). Avoidance theory of worry and generalized anxiety disorder. In R.G. Heimberg, C. L Turk \& D. S. Mennin (Eds.), Generalized anxiety disorder: Advances in research and practice (pp. 77-108). New York, NY: Guilford Press.

Borkovec, T. D., Hazlet-Stevens, H., \& Diaz, M. L. (1999). The role of positive beliefs about worry in generalized anxiety disorder and its treatment. Clinical Psychology and Psychotherapy, 6, 126-138. doi:10.1002/(SICI)1099-0879(199905)6:2<126::AID-

\section{CPP193>3.0.CO;2-M}

Borkovec, T. D., \& Hu, S. (1990). The effect of worry on cardiovascular response to phobic imagery. Behaviour Research and Therapy, 28, 69-73. doi:10.1016/0005-7967(90)90056- 
Borkovec, T. D., Ray, W. J., \& Stöber, J. (1998). Worry: A cognitive phenomenon intimately linked to affective, physiological, and interpersonal behavioral processes. Cognitive Therapy and Research, 22, 561-576. doi:10.1023/A:1018790003416

Borkovec, T. D., \& Roemer, L. (1995). Perceived functions of worry among generalized anxiety disorder subjects: Distraction from more emotionally distressing topics? Journal of Behavior Therapy and Experimental Psychiatry, 26, 25-30. doi:10.1016/00057916(94)00064-S

Bradley, M. M. \& Lang, P. J. (2007). Emotion and motivation. In J. T. Cacioppo, L. G. Tassinary \& G. G. Berntson (Eds.), Handbook of psychophysiology (3rd ed., pp. 581-607). New York, NY: Cambridge University Press.

Brain-Body Center. (2007a). CardioBatch [Computer software]. Chicago, IL: University of Illinois at Chicago.

Brain-Body Center. (2007b). CardioEdit [Computer software]. Chicago, IL: University of Illinois at Chicago.

Brans, K., Koval, P., Verdyn, P., Lim, Y. L., \& Kuppens, P. (2013). The regulation of negative and positive affect in daily life. Emotion, 13, 926-939. doi: 10.1037/a0032400

Brans, K., \& Verduyn, P. (2014). Comparing determinants of emotion intensity and duration: Appraisals and regulation strategies. PLOS ONE, 9, 1-13.

Braithwaite, J. J., Watson, D. G., Jones, R., \& Rowe, M. (2013). A guide for analysing electrodermal activity (EDA) and skin conductance responses (SCRs) for physiological experiments (Technical Report No. 2). Selective Attention \& Awareness Laboratory (SAAL) Behavioural Brain Sciences Centre, University of Birmingham, UK. Retrieved 
from http://www.biopac.com/manuals/EDA\%20SCR\%20Analysis.pdf

Brosschot, J. F., Gerin, W., \& Thayer, J. F. (2006). The perseverative cognition hypothesis: A review of worry, prolonged stress-related physiological activation and health. Journal of Psychosomatic Research, 60, 113-124. doi:10.1016/j.jpsychores.2005.06.074

Brown, T. A., \& Barlow, D. H. (2014). Anxiety and related disorders interview schedule for DSM-5 (ADIS-5) - Lifetime version. New York, NY: Oxford University Press.

Brown, T. A., Chorpita, B. F., \& Barlow, D. H. (1998). Structured relationships among dimensions of the DSM-IV anxiety and mood disorders and dimensions of negative affect, positive affect, and autonomic arousal. Journal of Abnormal Psychology, 107, 179-192. doi: 10.1037/0021-843X.107.2.179

Bylsma, L. M., Morris, B. H., \& Rottenberg, J. (2008). A meta-analysis of emotional reactivity in major depressive disorder. Clinical Psychology Review, 28, 676-691. doi:10.1016/j.cpr.2007.10.001

Bylsma, L. M., Taylor-Clift, A., \& Rottenberg, J. (2011). Emotional reactivity to daily events in major and minor depression. Journal of Abnormal Psychology, 120, 155-167. doi:10.1037/a0021662

Campbell-Sills, L., Ellard, K. K., \& Barlow, D. H. (2014). Emotion regulation in anxiety disorders. In J. J. Gross (Ed.), Handbook of emotion regulation ( $2^{\text {nd }}$ edition). New York, NY: Guilford Press.

Catanzaro, S. J., \& Mearns, J. (1990). Measuring generalized expectancies for negative mood regulation: Initial scale development and implications. Journal of Personality Assessment, 54, 546-563. doi:10.1207/s15327752jpa5403\&4_11

Carl, J. R., Fairholme, C. P., Gallagher, M. W., Thompson-Hollands, J., \& Barlow, D. H. (2014). 
The effects of anxiety and depressive symptoms on daily positive emotion regulation. Journal of Psychopathology and Behavioral Assessment, 36, 224-236. doi:

$10.1007 / \mathrm{s} 10862-013-9387-9$

Chalmers, J. A., Quintana, D. S., Abbott, M. J., \& Kemp, A. H. (2014). Anxiety disorders are associated with reduced heart rate variability: A meta-analysis. Frontiers in Psychiatry, 5, 80. doi:10.3389/fpsyt.2014.00080

Cisler, J. M., Olatunji, B. O., Feldner, M. T., \& Forsyth, J. P. (2010). Emotion regulation and the anxiety disorders: An integrated review. Journal of Psychopathology and Behavioral Assessment, 32, 68-82. doi: 10.1007/s 10862-009-9161-1

Clark, D. A., \& Beck, A. T. (2010). Cognitive therapy of anxiety disorders: Science and practice. New York, NY: Guilford Press.

Coles, M. E., Turk, C. L., \& Heimberg, R. G. (2007). Memory bias for threat in generalized anxiety disorder: The potential importance of stimulus relevance. Cognitive Behaviour Therapy, 36, 65-73. doi: 10.1080/16506070601070459

Connor, K. M., Davidson, J. R. T., Churchill, L. E., Sherwood, A., Foa, E. B., \& Weisler, R. H. (2000). Psychometric properties of the Social Phobia Inventory (SPIN): A new self-rating scale. British Journal of Psychiatry, 176, 379-386. doi: 10.1192/bjp.176.4.379

Cook, E. W., Melamed, B. G., Cuthbert, B. N., McNeil, D. W., \& Lang, P. J. (1988). Emotional imagery and the differential diagnosis of anxiety. Journal of Consulting and Clinical Psychology, 56, 734-740. doi: 10.1037/0022-006X.56.5.734

Crawford, J. R., \& Henry, J. D. (2004). The Positive and Negative Affect Schedule (PANAS): Construct validity, measurement properties and normative data in a large non-clinical sample. British Journal of Clinical Psychology, 43, 245-265. 
doi:10.1348/0144665031752934

Csikszentmihalyi, M., \& Larson, R. (1987). Validity and reliability of the experience sampling method. Journal of Nervous and Mental Disease, 175, 526-536. doi:10.1097/00005053198709000-00004

Curran, P. J., \& Bauer, D. J. (2011). The disaggregation of within-person and between-person effects in longitudinal models of change. Annual Review of Psychology, 62, 583-619. doi: 10.1146/annurev.psych.093008.100356

Davey, G. C. L., \& Meeten, F. (2016). The perseverative worry bout: A review of cognitive, affective and motivational factors that contribute to worry perseveration. Biological Psychology. Advance online publication. doi: 10.1016/j.biopsycho.2016.04.003

Davey, G. C. L., Tallis, F., \& Capuzzo, N. (1996). Beliefs about the consequences of worrying. Cognitive Therapy and Research, 20, 499-520. doi:10.1007/BF02227910

Dawson, M. E., Schell, A. M., \& Filion, D. L. (2007). The electrodermal system. In J. T. Cacioppo, L. G. Tassinary \& G. G. Berntson (Eds.), Handbook of psychophysiology (3rd ed., pp. 159-181). New York, NY: Cambridge University Press.

Decker, M. L., Turk, C. L., Hess, B., \& Murray, C. E. (2008). Emotion regulation among individuals classified with and without generalized anxiety disorder. Journal of Anxiety Disorders, 22, 485-494. doi: 10.1016/j.janxdis.2007.04.002

Diefenbach, G. J., Stanley, M. A., \& Beck, J. G. (2001). Worry content reported by older adults with and without generalized anxiety disorder. Aging and Mental Health, 5, 269-274. doi:10.1080/13607860120065069

Diener, E., \& Emmons, R. A. (1984). The independence of positive and negative affect. Journal of Personality and Social Psychology, 47, 1105-1117. doi:10.1037/0022-3514.47.5.1105 
Dugas, M. J., Gagnon, F., Ladouceur, R., \& Freeston, H. (1998). Generalized anxiety disorder: A preliminary test of a conceptual model. Behaviour Research and Therapy, 36, 215-226. doi: 10.1016/S00005-7967(97)00070-3

Dugas, M. J., \& Koerner, N. (2005). Cognitive-behavioral treatment for generalized anxiety disorder: Current status and future directions. Journal of Cognitive Psychotherapy, 19, 61-82. doi:10.1891/088983905780907252

Dugas, M. J., \& Robichaud, M. (2007). Cognitive-behavioral treatment for generalized anxiety disorder: From science to practice. New York, NY: Routledge.

Dupuy, J.-B., Beaudoin, S., Rhéaume, J., Ladouceur, R., \& Dugas, M. J. (2001). Worry: Daily self-report in clinical and non-clinical populations. Behaviour Research and Therapy, 39, 1249-1255. doi:10.1016/S0005-7967(01)00011-0

Eagleson, C., Hayes, S., Mathews, A., Perman, G., \& Hirsch, C. R. (2016). The power of positive thinking: Pathological worry is reduced by thought replacement in generalized anxiety disorder. Behaviour Research and Therapy, 78, 13-18.

doi:10.1016/j.brat.2015.12.017

Eaton, W. W., Smith, C., Ybarra, M., Muntaner, C., \& Tien, A. (2004). Center for Epidemiologic Studies Depression Scale: Review and revision (CESD and CESD-R). In M. E. Maruish (Ed.), The use of psychological testing for treatment planning and outcomes assessment:

Vol. 3 (Instruments for adults) (pp. 363-377). Mahwah, NJ: Lawrence Erlbaum Associates.

Ebner-Priemer, U. W., \& Trull, T. J. (2009). Ecological momentary assessment of mood disorders and mood dysregulation. Psychological Assessment, 21, 463-475. doi: $10.1037 / \mathrm{a} 0017075$ 
Eid, M., \& Diener, E. (1999). Intraindividual variability in affect: Reliability, validity, and personality characteristics. Journal of Personality and Social Psychology, 76, 662-676. doi: $10.1037 / 0022-3514.76 .4 .662$

Eisner, L. R., Johnson, S. L., \& Carvers, C. S. (2009). Positive affect regulation in anxiety disorders. Journal of Anxiety Disorders, 23, 645-649. doi:10.1016/j.janxdis.2009.02.001.

Ellard, K. K., Farchione, T. J., \& Barlow, D. H. (2012). Relative effectiveness of emotion induction procedures and the role of personal relevance in a clinical sample: A comparison of film, images, and music. Journal of Psychopathology and Behavioral Assessment, 34, 232-243. doi:10.1007/s10862-011-9271-4

Farmer, A. S., \& Kashdan, T. B. (2013). Affective and self-esteem instability in the daily lives of people with generalized social anxiety disorder. Clinical Psychological Science, 2, 187201. doi: $10.1177 / 2167702613495200$

Feldman Barrett, L.., \& Barrett, D. J. (2001). An introduction to computerized experience sampling in psychology. Social Science Computer Review, 19, 175-185.

Fischer, A., Manstead, A. S. R., Evers, C., Timmers, M., \& Valk, G. (2004). Motives and norms underlying emotion regulation. In P. Philippot, \& R. S. Feldman (Eds.), The regulation of emotion (pp. 189-214). London, UK: Lawrence Erlbaum Associates.

Fisher, A. J., Granger, D. A., \& Newman, M. G. (2010). Sympathetic arousal moderates selfreported physiological arousal symptoms at baseline and physiological flexibility in response to a stressor in generalized anxiety disorder. Biological Psychology, 83, 191200. doi: 10.1016/j.biopsycho.2009.12.007

Fisher, A. J., \& Newman, M. G. (2013). Heart rate and autonomic response to stress after experimental induction of worry versus relaxation in healthy, high-worry, and 
generalized anxiety disorder individuals. Biological Psychology, 93, 65-74. doi:

10.1016/j.biopsycho.2013.01.012

Flett, G. L., \& Hewitt, P.L. (1995). Criterion validity and psychometric properties of the Affect Intensity Measure in a psychiatric sample. Personality and Individual Differences, 19, 585-591. doi: 10.1016/0191-8869(95)00089-O

Fracalanza, K., Koerner, N., \& Antony, M.M. (2014). Testing a procedural variant of written imaginal exposure for generalized anxiety disorder. Journal of Anxiety Disorders, 28, 559-569. doi:10.1016/j.janxdis.2014.05.011

Fredrickson, B. L. (1998). What good are positive emotions? Review of General Psychology, 2, 300-319. doi: 10.1037/1089-2680.2.3.300

Fresco, D. M., Mennin, D. S., Heimberg, R. G., \& Ritter, M. (2013). Emotion regulation therapy for generalized anxiety disorder. Cognitive and Behavioral Practice, 20, 282-300. doi: 10.1016/j.cbpra.2013.02.001

Fresco, D. M., Mennin, E. S., Heimberg, R. G., \& Turk, C. L. (2003). Using the Penn State Worry Questionnaire to identify individuals with generalized anxiety disorder: A receiver operating characteristic analysis. Journal of Behavior and Experimental Psychiatry, 34, 283-291. doi: 10.1016/j.jbtep.2003.09.001

Gable, S. L., Reis, H. T., \& Elliot, A. J. (2000). Behavioral activation and inhibition in everyday life. Journal of Personality and Social Psychology, 78, 1135-1149. doi: 10.1037/00223514.78.6.1135

Gratz, K. L., \& Roemer, L. (2004). Multidimensional assessment of emotion regulation and dysregulation: Development, factor structure, and initial validation of the Difficulties in Emotion Regulation Scale. Journal of Psychopathology and Behavioural Assessment, 26, 
41-54. doi: 10.1007/s10862-008-9102-4.

Gratz, K. L., \& Tull, M. T. (2010). Emotion regulation as a mechanism of change in acceptanceand mindfulness-based treatments. In R. A. Baer (Ed.), Assessing mindfulness and acceptance processes in clients (pp. 107-133). Oakland, CA: New Harbinger Publications.

Gray, J. A. (1982). The neuropsychology of anxiety: An enquiry into the functions of the septohippocampal system. New York, NY: Oxford University Press.

Gross, J. J. (1998a). Antecedent- and response-focused emotion regulation: Divergent consequences for experience, expression, and physiology. Journal of Personality and Social Psychology, 74, 224-237. doi:10.1037/0022-3514.74.1.224

Gross, J. J. (1998b). The emerging field of emotion regulation: An integrative review. Review of General Psychology, 2, 271-299. doi: 10.1037/1089-2680.2.3.271

Gross, J. J. (2014). Handbook of emotion regulation ( $2^{\text {nd }}$ edition). New York, NY: Guilford Press.

Gross, J. J., \& John, O. P. (1997). Revealing feelings: Facets of emotional expressivity in selfreports, peer ratings, and behavior. Journal of Personality and Social Psychology, 72, 435-448. doi: 10.1037/0022-3514.72.2.435

Gross, J. J., \& John, O. P. (2003). Individual differences in two emotion regulation processes: Implications for affect, relationships and well-being. Journal of Personality and Social Psychology, 85, 348-362. doi: 10.1037/0022-3514.85.2.348

Gross, J. J., \& Muñoz, R. F. (1995). Emotion regulation and mental health. Clinical Psychology: Science and Practice, 2, 151-164. doi: 10.1111/j.1468-2850.1995.tb00036.x

Gross, J. J., \& Thompson, R. A. (2007). Emotion regulation: Conceptual foundations. In J. J. 
Gross (Ed.), Handbook of emotion regulation (pp. 3-24). New York, NY: Guilford Press.

Gruber, J., Kogan, A., Quoidbach, J., \& Mauss, I. B. (2013). Happiness is best kept stable:

Positive emotion variability is associated with poorer psychological health. Emotion, 13, 1-6. doi: $10.1037 / \mathrm{a} 0030262$

Gueorguieva, R., \& Krystal, J. H. (2004). Move over ANOVA: Progress in analyzing repeatedmeasures data and its reflection in papers published in the Archives of General Psychiatry. Archives of General Psychiatry, 61, 310-317. doi:

10.1001/archpsyc.61.3.310.

Hall, D. P., Sing, H. C., \& Romanowski, A. J. (1991). Identification and characterization of greater mood variance in depression. American Journal of Psychiatry, 148, 1341-1345. doi:10.1176/ajp.148.10.1341

Hancock, G.R., \& Klockars, A. J. (1996). The quest for $\alpha$ : Developments in multiple comparison procedures in the quarter century since Games (1971). Review of Educational Research, 66, 269-306. doi: 10.3102/00346543066003269

Hanrahan, F., Field, A. P., Jones, F. W., \& Davey, G. C. L. (2013). A meta-analysis of cognitive therapy for worry in generalized anxiety disorder. Clinical Psychology Review, 33, 120132. doi:10.1016/j.cpr.2012.10.008

Hayes, S., Hirsch, C., \& Mathews, A. (2008). Restriction of working memory capacity during worry. Journal of Abnormal Psychology, 117, 712-717. doi:10.1037/a0012908

Haynes, S. N., \& O'Brien, W. H. (1990). Functional analysis in behavior therapy. Clinical Psychology Review, 10, 649-668. Doi: 10.1016/0272-7358(90)90074-K

Hazlett-Stevens, H., \& Craske, M. G. (2003). The catastrophizing worry process in generalized anxiety disorder: A preliminary investigation of an analog population. Behavioural and 
Cognitive Psychotherapy,31, 387-401. doi:10.1017/S1352465803004016

Hektner, J. M., Schmidt, J. A., \& Csikszentmihalyi, M. (2007). Experience sampling method: Measuring the quality of everyday life. Thousand Oaks, CA: Sage Publications, Inc.

Higgins, E. T. (1997). Beyond pleasure and pain. American Psychologist, 52, 1280-1300. doi:10.1037/0003-066X.52.12.1280

Hirt, E. R., \& McCrea, S. M. (2000). Beyond hedonism: Broadening the scope of affect regulation. Psychological Inquiry, 11, 180-183.

Hoehn-Saric, R., \& McLeod, D. R. (2000). Anxiety and arousal: Physiological changes and their perception. Journal of Affective Disorders, 61, 217-224. doi: 10.1016/S01650327(00)00339-6

Hoehn-Saric, R., McLeod, D. R., Funderburk, F., \& Kowalski, P. (2004). Somatic symptoms and physiologic responses in generalized anxiety disorder and panic disorder: An ambulatory monitor study. Archives of General Psychiatry, 61, 913-921. doi:10.1001/archpsyc.61.9.913. doi:10.1001/archpsyc.61.9.913

Hofmann, S. G., Moscovitch, D. A., \& Kim, H.-J. (2006). Autonomic correlates of social anxiety and embarrassment in shy and non-shy individuals. International Journal of Psychophysiology, 61, 134-142. doi: 10.1016/j.jpssycho.2005.09.003

Hodgson, R., \& Rachman, S. (1974). II. desynchrony in measures of fear. Behaviour Research and Therapy, 12, 319-326. doi:10.1016/0005-7967(74)90006-0

Houben, M., Van Den Noortgate, W., \& Kuppens, P. (2015). The relation between short-term emotion dynamics and psychological well-being: A meta-analysis. Psychological Bulletin, 141, 901-930. doi: 10.1037/a0038822

Huppert, J. D. \& Foa, E. B. (2004). Maintenance mechanisms in social anxiety: An integration of 
cognitive biases and emotional processing theory. In J. Yiend (Ed.), Cognition, emotion and psychopathology: Theoretical, empirical and clinical directions (pp. 213-231). Cambridge, UK: Cambridge University Press.

Jacob, G. A., Hellstern, K., Ower, N., Pillmann, M., Scheel, C. N., ..., Lieb, K. (1999). Emotional reactions to standardized stimuli in women with borderline personality disorder: Stronger negative affect, but no differences in reactivity. Journal of Nervous and Mental Disease, 197, 808-815. doi: 10.1097/NMD.0b013e3181bea44d

Jahng, S., Wood, P. K., \& Trull, T. J. (2008). Analysis of affective instability in ecological momentary assessment: Indices using successive difference and group comparison via multilevel modeling. Psychological Methods, 13,354-375. doi: 10.1037/a0014173

Jennings, J., Kamarck, T., Stewart, C., Eddy, M., \& Johnson, P. (1992). Alternate cardiovascular baseline assessment techniques: Vanilla or resting baseline. Psychophysiology, 29, 742750. doi: 10.1111/j.1469-8986.1992.tb02052.x

Johnston, W. M. \& Davey, G. C. L. (1997). The psychological impact of negative TV news bulletins: The catastrophizing of personal worries. British Journal of Psychology, 88, 8597. doi: 10.1111/j.2044-8295.1997.tb02622.x

Kashdan, T. B. (2007). Social anxiety spectrum and diminished positive experiences: Theoretical synthesis and meta-analysis. Clinical Psychology Review, 27, 348-365. doi:10.1016/j.cpr.2006.12.003

Kashdan, T. B., \& Steger, M.F. (2006). Expanding the topography of social anxiety: An experience-sampling assessment of positive emotions, positive events, and emotion suppression. Psychological Science, 17, 120-128. doi: 10.1111/j.1467-9280.2006.01674.x

Klonsky, E. D. (2007). The functions of deliberate self-injury: A review of the evidence. Clinical 
Psychology Review, 27, 226-239. doi:10.1016/j.cpr.2006.08.002

Koval, P., Butler, E. A., Hollenstein, T., Lanteigne, D., \& Kuppens, P. (2015). Emotion regulation and the temporal dynamics of emotions: Effects of cognitive reappraisal and expressive suppression on emotional inertia. Cognition and Emotion, 29, 831-851. doi: $10.1080 / 02699931.2014 .948388$

Koval, P. \& Kuppens, P. (2012). Changing emotion dynamics: Individual differences in the effect of anticipatory social stress on emotional inertia. Emotion, 12, 256-267. doi: $10.1037 / \mathrm{a} 0024756$

Koval, P., Kuppens, P., Allen, N. B., \& Sheeber, L. (2012). Getting stuck in depression: The roles of rumination and emotional inertia. Cognition and Emotion, 26, 1412-1427. doi:10.1080/02699931.2012.667392

Koval, P., Pe, M. L., Meers, K., \& Kuppens, P. (2013). Affect Dynamics in Relation to Depressive Symptoms: Variable, Unstable or Inert? Emotion, 13, 1132-1141. doi: $10.1037 / \mathrm{a} 0033579$

Kreibig, S. D. (2010). Autonomic nervous system activity in emotion: A review. Biological Psychology, 84, 394-342. doi:10.1016/j.biopsycho.2010.03.010

Kuo, J. R., \& Linehan, M.M. (2009). Disentangling emotion processes in borderline personality disorder: Physiological and self-reported assessment of biological vulnerability, baseline intensity, and reactivity to emotionally evocative stimuli. Journal of Abnormal Psychology, 118, 531-544. doi: 10.1037/a0016392

Kuo, J. R., Neacsiu, A. D., Fitzpatrick, S., \& MacDonald, D. E. (2013). A methodological examination of emotion inductions in borderline personality disorder: A comparison of standardized versus idiographic stimuli. Journal of Psychopathology and Behavioral 
Assessment, 36, 155-154. doi: 10.1007/s10862-013-9378-x

Kuppens, P., Allen, N. B., \& Sheeber, L. B. (2010). Emotional inertia and psychological adjustment. Psychological Science, 21, 984-991. doi: 10.1177/0956797610372634

Lane, R. D., Quinlan, D. M., Schwartz, G. E., Walker, P. A., \& Zeitlin, S. B. (1990). The Levels of emotional awareness scale: A cognitive-developmental measure of emotion. Journal of Personality Assessment, 55, 124-134. doi: 10.1080/00223891.1990.9674052

Lang, P. J. (1968). Fear reduction and fear behavior: Problems in treating a construct. In J. M. Shlien (Ed), Research in psychotherapy (pp. 90-102). Washington, DC: American Psychological Association.

Lang, P. J. (1971) The application of psychophysiological methods to the study of psychotherapy and behavior modification. In A. E. Bergin and S. L. Garfield (Eds.), Handbook of Psychotherapy and Behavior Change (pp. 75-125). New York, NY: John Wiley and Sons.

Lang, P. J., Bradley, M. M., \& Cuthbert, B. N. (1998). Emotion, motivation, and anxiety: Brain mechanisms and psychophysiology. Biological Psychiatry, 44, 1248-1263. doi: $10.1016 / \mathrm{S} 0006322398002753$

Lang, P. J., Greenwald, M. K., Bradley, M. M., \& Hamm, A. O. (1993). Looking at pictures: affective, facial, visceral, and behavioral reactions. Psychophysiology 30, 261-273. doi: 10.1111/j.1469-8986.1993.tb03352.x

Lang, P. J., Levin, D. N., Miller, G. A., \& Kozak, M. J. (1983). Fear behavior, fear imagery, and the psychophysiology of emotion: The problem of affective response integration. Abnormal Psychology, 92, 276-306. doi: 10.1037/0021-843X.92.3.276

Langston, C. A. (1994). Capitalizing on and coping with daily-life events: Expressive responses 
to positive events. Journal of Personality and Social Psychology, 67, 1112-1125. doi: 10.1037/0022-3514.67.6.1112

Larsen, J. T., Berntson, G. G., Poehlmann, K. M., Ito, T. A., \& Cacioppo, J. T. (2008). The psychophysiology of emotion. In M. Lewis, J. M., Haviland-Jones, \& L. Feldman Barrett (Eds.), Handbook of emotions (p. 180-195). New York, NY: Guilford Press.

Larsen, R. J. (1984). Theory and measurement of affect intensity as an individual difference characteristic. Dissertation Abstracts International, 85, 2297B.

Larsen, R. J. (2000). Toward a science of mood regulation. Psychological Inquiry, 11, 129-141. doi:10.1207/S15327965PLI1103_01

Larsen, R. J., \& Deiner, E. (1987). Affect intensity as an individual difference characteristic: A review. Journal of Research in Personality, 21, 1-39. doi: 10.1037/0022-3514.67.6.1112

Larsen, R. J., Deiner, E, \& Emmons, R. A. (1986). Affect intensity and reactions to daily life events. Journal of Personality and Social Psychology, 51, 803-814. doi: 10.1037/00223514.51 .4 .803

Levy, M. N. (1984). Cardiac sympathetic-parasympathetic interactions. Federation Proceedings, $43,2598-2602$.

Linehan, M. (1993). Cognitive-behavioral treatment of borderline personality disorder. New York, NY: Guilford Press.

Llera, S. J., \& Newman, M. G. (2010). Effects of worry on physiological and subjective reactivity to emotional stimuli in generalized anxiety disorder and nonanxious control participants. Emotion, 10, 640-650. doi: 10.1037/a0019351

Llera, S. J., \& Newman, M. G. (2014). Rethinking the role of worry in generalized anxiety disorder: Evidence supporting a model of emotional contrast avoidance. Behavior 
Therapy, 45, 283-299. doi: 10.1016/j.beth.2013.12.011

Lynch, T. R., Chapman, A. L., Rosenthal, M. Z., Kuo, J. R., \& Linehan, M. M. (2006). Mechanisms of change in dialectical behavior therapy: Theoretical and empirical observations. Journal of Clinical Psychology, 62, 459-480. doi:10.1002/jclp.20243

Lyonfields, J. D., Borkovec, T. D., \& Thayer, J. F. (1995). Vagal tone in generalized anxiety disorder and effects of aversive imagery and worrisome thinking. Behavior Therapy, 26, 457-466. doi: 1016/S0005-7894(05)80094-2

Macatee, R. J., \& Cougle, J. R. (2013). The roles of emotional reactivity and tolerance in generalized, social, and health anxiety: A multimethod exploration. Behavior Therapy, 44, 39-59. doi: 10.1016/j.beth.2012.05.006

Marco, C. A., Neale, J. M., Schwartz, J. E., Shiffman, S., \& Stone, A. A. (1999). Coping with daily events and short-term mood changes: An unexpected failure to observe effects of coping. Journal of Consulting and Clinical Psychology, 67, 755-764. doi: 10.1037/0022006X.67.5.755

Marks, I. M., \& Huson, J. (1973). Physiological aspects of neutral and phobic imagery: Further observations. British Journal of Psychiatry, 122, 567-572. doi:10.1192/bjp.122.5.567

Martin, L. L., \& Davies, B. (1998). Beyond hedonism and associationism: A configural view of the role of affect in evaluation, processing, and self-regulation. Motivation and Emotion, 22, 33-51. doi:10.1023/A:1023092508471

Mathews, A. \& Klug, F. (1993). Emotionality and interference with color-naming in anxiety. Behaviour Research and Therapy, 31, 57-62. doi: 10.1016/0005-7967(93)90043-T

Mathews, A., \& MacLeod, C. (2005). Cognitive vulnerability to emotional disorders. Annual Review of Clinical Psychology, 1, 167-195. doi: 


\subsection{6/annurev.clinpsy.1.102803.143916}

McConville, C., \& Cooper, C. (1996). Mood variability and the intensity of depressive states. Current Psychology, 14, 329-338. doi: 10.1007/BF02686921

McCraty, R., Atkinson, M., Tiller, W. A., Rein, G., \& Watkins, A. D. (1995). The effects of emotions in short-term power spectrum analysis of heart rate variability. American Journal of Cardiology, 76, 1089-1093. doi: 10.1016/S0002-9149(99)80309-9

McLaughlin, K. A., Mennin, D. S., \& Farach, F. J. (2007). The contributory role of worry in emotion generation and dysregulation in generalized anxiety disorder. Behaviour Research and Therapy, 45, 1735-1752. doi:10.1016/j.brat.2006.12.004

McLeod, D. R., \& Hoehn-Saric, R. (1993). Perception of physiological changes in normal and pathological anxiety. In R. Hoehn-Saric \& D. R. McLeod (Eds.), Biology of anxiety disorders (pp. 223-243). Arlington, VA: American Psychiatric Press.

McLeod, D. R., Hoehn-Saric, R., \& Stefan, R. L. (1986). Somatic symptoms of anxiety: Comparison of self-report and physiological measures. Biological Psychiatry, 21, 301310. doi:10.1016/0006-3223(86)90051-X

McTeague, L. M., \& Lang, P. J. (2012). The anxiety spectrum and the reflex physiology of defense: From circumscribed fear to broad distress. Depression and Anxiety, 29, 264-281. doi:10.1002/da.21891

Mees, U., \& Schmitt, A. (2008). Goals of action and emotional reasons for action. A modern version of the theory of ultimate psychological hedonism. Journal for the Theory of Social Behaviour, 38, 157-178. doi:10.1111/j.1468-5914.2008.00364.x

Mennin, D. S., \& Fresco, D. M. (2015). Advancing emotion regulation perspectives on psychopathology: The challenges of distress disorders. Psychological Inquiry, 26, 80-92. 
doi: 10.1080/1047840X.2015.969624

Mennin, D. S, Heimberg, R. G., Turk, C. L., \& Fresco, D. M. (2002). Applying an emotion regulation framework to integrative approaches to generalized anxiety disorder. Clinical Psychology: Science and Practice, 9, 85-90. doi: 10.1093/clipsy.9.1.85

Mennin, D. S., Heimberg, R. G., Turk, C. L., \& Fresco, D. M. (2005). Preliminary evidence for an emotion dysregulation model of generalized anxiety disorder. Behaviour Research and Therapy, 43, 1281-1310. doi:10.1016/j.brat.2004.08.008

Mennin, D. S., Holaway, R. M., Fresco, D. M., Moore, M. T., \& Heimberg, R. G. (2007). Delineating components of emotion and its dysregulation in anxiety and mood psychopathology. Behavior Therapy, 38, 284-302. doi: 10.1016/j.beth.2006.09.001

Mennin, D. S., McLaughlin, K. A., \& Flanagan, T. J. (2009). Emotion regulation deficits in generalized anxiety disorder, social anxiety disorder, and their co-occurrence. Journal of Anxiety Disorders, 23, 866-871. doi: 10.1016/j.janxdis.2009.04.006

Mennin, D. S., Turk, C. L., Heimberg, R. G., \& Carmin, C. N. (2004). Regulation of emotion in generalized anxiety disorder. In M. A. Reinecke \& D. A. Clark (Eds.), Cognitive therapy across the lifespan: Evidence and practice (pp. 60-89). Cambridge, UK: Cambridge University Press.

Meyer, T. J., Miller, M. L., Metzger, R. K., \& Borkovec, T. D. (1990). Development and validation of the Penn State Worry Questionnaire. Behaviour Research and Therapy, 28, 486-495. doi:10.1016/0005-7967(90)90135-6

Miller, G. A., Levin, D. N., Kozak, M. J., Cook, E. W., McLean, A., \& Lang, P. J. (1987). Individual differences in imagery and the psychophysiology of emotion. Cognition and Emotion, 1, 367-390. doi:10.1080/02699938708408058 
Miller, M. W., Patrick, C. J., \& Levenston, G. K. (2002). Affective imagery and the startle response: Probing mechanisms of modulation during pleasant scenes, personal experiences, and discrete negative emotions. Psychophysiology, 4, 519-529. doi: $10.1017 / \mathrm{S} 0048577202394095$

Moberly, N. J., \& Watkins, E. R. (2008). Ruminative self-focus and negative affect: An experience sampling study. Journal of Abnormal Psychology, 117, 314-323. doi: 10.1037/0021-843X.117.2.314

Moscovitch, D. A., Suvak, M. K., \& Hofmann, S. G. (2010). Emotional response patterns during social threat in individuals with generalized social anxiety disorder and non-anxious controls. Journal of Anxiety Disorders, 24, 785-791. doi:10.1016/j.janxdis.2010.05.013

Brain-Body Center. (2007b). CardioEdit [Computer software]. Chicago, IL: University of Illinois at Chicago.

movisens GmbH. (2015). movisensXS, Version 0.4.2437 [application]. Karlsruhe, Germany. Murray, G., Allen, N. B., \& Trinder, J. (2002). Longitudinal investigation of mood variability and the FFM: Neuroticism predicts variability in extended states of positive and negative affect. Personality and Individual Differences, 33, 1217-1228. doi: 10.1016/S01918869(01)00217-3

Neacsiu, A. D., Eberle, J. W., Kramer, R., Wiesmann, T., \& Linehan, M. M. (2014). Dialectical behavior therapy skills for transdiagnostic emotion dysregulation: A pilot randomized controlled trial. Behaviour Research and Therapy, 59, 40-51. doi:

10.1016/j.brat.2014.05.005

Newman, M. G., Zuellig, A. R., Kachin, K. E., Constantino, M. J., Prezeworski, A., Erickson T., \& Cashman-McGrath, L. (2002). Preliminary reliability and validity of the Generalized 
Anxiety Disorder Questionnaire-IV: A revised self-report diagnostic measure of generalized anxiety disorder. Behavior Therapy, 33, 215-233. doi:10.1016/S00057894(02)80026-0

Nezlek, J. B. (2012). Multilevel modeling of diary-style data. In M. R. Mehl \& T. S. Conner (Eds.), Handbook of research methods for studying daily life (pp. 357-383). New York, NY: Guilford Press.

Nezlek, J. B., \& Kuppens, P. (2008). Regulating positive and negative emotions in daily life. Journal of Personality, 76, 561-580. doi: 10.1111/j.1467-6494.2008.00496.x

Nolen-Hoeksema, S. (2000). The role of rumination in depressive disorders and mixed Anxiety/Depressive symptoms. Journal of Abnormal Psychology, 109, 504-511. doi:10.1037/0021-843X.109.3.504

Novick-Kline, P., Turk, C. L., Mennin, D. S., Hoyt, E. A., \& Gallagher. C. L. (2005). Level of emotional awareness as a differentiating variable between individuals with and without generalized anxiety disorder. Journal of Anxiety Disorders, 19, 557-572. doi: 10.1016/j.janxdis.2004.06.001

Orr, S. P., Pitman, R. K., Lasko, M. B., \& Herz, L. R. (1993). Psychophysiological assessment of posttraumatic stress disorder imagery in World War II and Korean combat veterans. Journal of Abnormal Psychology, 102, 152-159. doi: 10.1037/0021-843X.102.1.152

Parrott, W. G. (2001). Implications for dysfunctional emotions for understanding how emotions function. Review of General Psychology, 5, 180-186. doi: 10.1037/1089-2680.5.3.180

Pasyugina, I., Koval, P., De Leersnyder, J., Mesquita, B., \& Kuppens, P. (2015). Distinguishing between level and impact of rumination as predictors of depressive symptoms: An experience sampling study. Cognition and Emotion, 29, 736-746. doi: 
$10.1080 / 02699931.2014 .932755$

Peeters, F., Nicolson, N. A., Berkhof, J., Delespaul, P., \& deVries, M. (2003). Effects of daily events on mood states in major depressive disorder. Journal of Abnormal Psychology, 112, 203-211. doi: 10.1037/0021-843X.112.2.203

Pitman, R., Orr, S., Forgue, D., de Jong, J., \& Claiborn, J. (1987). Psychophysiologic assessment of posttraumatic stress disorder imagery in Vietnam combat veterans. Archives of General Psychiatry, 44, 970-975. doi:10.1001/archpsyc.1987.01800230050009

Pittig, A., Arch, J., Lam, C. W. R., \& Craske, M. G. (2013). Heart rate and heart rate variability in panic, social anxiety, obsessive-compulsive, and generalized anxiety disorders at baseline and in response to relaxation and hyperventilation. International Journal of Psychophysiology, 87, 19-27. doi: 10.1016/j.ijpsycho.2012.10.012

Porges, S. W. (1991). Vagal tone: An autonomic mediator of affect. In J. Garber \& K. A. Dodge (Eds.), The development of emotion regulation and dysregulation (pp. 111-128). New York, NY: Cambridge University Press.

Porges, S. W. (2007). The polyvagal perspective. Biological Psychology, 74, 116-143. doi: 10.1016/j.biopsycho.2006.06.009

Pruneti, C. A., Lento, R. M., Fante, C., Carrozzo, E., \& Fontana, F. (2010). Autonomic arousal and differential diagnosis in clinical psychology and psychopathology. Italian Journal of Psychopathology, 16, 43-52.

Radloff, L. S. (1977). The CES-D Scale: A self-report depression scale for research in the general population. Applied Psychology Measurement, 1, 385-401. doi: $10.1177 / 014662167700100306$

Raudenbush, S. W., \& Bryk, A. S. (2002). Hierarchical linear models: Applications and data 
analysis methods ( $2^{\text {nd }}$ ed.). Thousand Oaks, CA: Sage Publications.

Raudenbush, S. W., Bryk, A. S, \& Congdon, R. (2011). HLM7 for Windows [Computer software]. Skokie, IL: Scientific Software International, Inc.

Ree, M. J., French, D., MacLeod, C., \& Locke, V. (2008). Distinguishing cognitive and somatic dimensions of state and trait anxiety: Development and validation of the State-Trait Inventory for Cognitive and Somatic Anxiety (STICSA). Behavioural and Cognitive Psychotherapy, 36, 313-332. doi: 10.1017/S1352465808004232

Reis, H. T., \& Gable, S. L. (2000). Event-sampling and other methods for studying everyday experience. In H. T. Reis \& C. M. Judd (Eds.), Handbook of research methods in social and personality psychology (pp. 190-222). Cambridge, UK: Cambridge University Press.

Riediger, M., Wrzus, C., Schmiedek, F., Wagner, G. G., \& Lindenberger, U. (2011). Is seeking bad mood cognitively demanding? contra-hedonic orientation and working-memory capacity in everyday life. Emotion, 11, 656-665. doi:10.1037/a0022756

Roemer, L., Molina, S., \& Borkovec, T. D. (1997). An investigation of worry content among generally anxious individuals. Journal of Nervous and Mental Disease, 185, 314-319. doi:10.1097/00005053-199705000-00005

Roemer, L., \& Orsillo, S. M. (2002). Expanding our conceptualization of and treatment for generalized anxiety disorder: Integrating mindfulness/acceptance-based approaches with existing cognitive-behavioral models. Clinical Psychology: Science and Practice, 9, 5468. doi:10.1093/clipsy.9.1.54

Salters-Pedneault, K., Gentes, E., \& Roemer, L. (2007). The role of fear of emotion in distress, arousal, and cognitive interference following an emotional stimulus. Cognitive Behaviour Therapy, 36, 12-22. doi:10.1080/16506070600874281 
Salters-Pedneault, K., Roemer, L., Tull, M. T., Rucker, L., \& Mennin, D. S. (2006). Evidence of broad deficits in emotion regulation associated with chronic worry and generalized anxiety disorder. Cognitive Therapy and Research, 30, 469-480. doi: 10.1007/s10608006-9055-4

Sheehan, D. V. (2014). The Mini-International Neuropsychiatric Interview, Version 7.0 for DSM-5 (M.I.N.I 7.0). Medical Outcomes Systems.

Sheehan, D.V., Lecrubier, Y., Harnett-Sheehan, K., Janavs, J., Weiller, E., Bonara, L.I., ..., Dunbar, G.C. (1997). Reliability and validity of the M.I.N.I. International Neuropsychiatric Interview (M.I.N.I.): According to the SCID-P. European Psychiatry, $12,232-241$.

Shiota, M. N., Newfeld, S. L., Yeung, W. H., Moser, S. F., \& Perea, E. F. (2011). Feeling good: Autonomic nervous system responding in five positive emotions. Emotion, 11, 13681378. doi: $10.1037 / \mathrm{a} 0024278$

Solhan, M. B., Trull, T. J., Jahng, S., \& Wood, P. K. (2009). Clinical assessment of affective instability: Comparing EMA indices, questionnaire reports, and retrospective recall. Psychological Assessment, 21, 425-436. doi:10.1037/a0016869

Stapinski, L. A., Abbott, M. J., \& Rapee, R. M. (2010). Evaluating the cognitive avoidance model of generalised anxiety disorder: Impact of worry on threat appraisal, perceived control and anxious arousal. Behaviour Research and Therapy, 48, 1032-1040. doi:10.1016/j.brat.2010.07.005

Stein, K. F. (1996). Affect instability in adults with a borderline personality disorder. Archives of Psychiatric Nursing, 10, 32-40. doi: 10.1016/S0883-9417(96)80084-7

Stöber, J., \& Joormann, J. (2001). Worry, procrastination, and perfectionism: Differentiating 
amount of worry, pathological worry, anxiety, and depression. Cognitive Therapy and Research, 25, 49-60. doi: 10.1023/A:1026474715384

Stöber, J., Tepperwien, S., \& Staak, M. (2000). Worrying leads to reduced concreteness of problem elaborations: Evidence for the avoidance theory of worry. Anxiety, Stress and Coping, 13, 217-227. doi:10.1080/10615800008415725

Stone, A. A., Schwartz, J. E. Neale, J. M., Shiffman, S., Marco, C.A., Hickcox, M., ..., Cruise, L. J. (1998). A comparison of coping assessed by ecological momentary assessment and retrospective recall. Journal of Personality and Social Psychology, 74, 1670-1680. doi: $10.1037 / 0022-3514.74 .6 .1670$

Stone, A., A. \& Shiffman, S. (1994). Ecological momentary assessment (EMA) in behavioral medicine. Annals of Behavioral Medicine, 16, 199-202.

Strauss, G. P., \& Allen, D. N. (2008). Emotional intensity and categorisation ratings for emotional and nonemotional words. Cognition and Emotion, 22, 114-133. doi: $10.1080 / 02699930701319154$

Suls, J., Green, P., \& Hillis, S. (1998). Emotional reactivity to everyday problems, affective inertia, and neuroticism. Personality and Social Psychology Bulletin, 24, 127-136. doi: $10.1177 / 0146167298242002$

Suls, J., \& Martin, R. (2005). The daily life of the garden-variety neurotic: Reactivity, stressor exposure, mood spillover, and maladaptive coping. Journal of Personality, 73, 1-25. doi: $10.1111 / \mathrm{j} .1467-6494.2005 .00356 . \mathrm{x}$

Tabachnick, B. G., \& Fidell, L. S. (2007). Using multivariate statistics ( $5^{\text {th }}$ edition). Bostin, MA: Allyn \& Bacon.

Tamir, M. (2005). Don't worry, be happy? neuroticism, trait-consistent affect regulation, and 
performance. Journal of Personality and Social Psychology, 89, 449-461.

doi:10.1037/0022-3514.89.3.449

Tamir, M. (2015). Why do people regulate their emotions? A taxonomy of motives in emotion regulation. Personality and Social Psychology Review. Advance online publication. doi: $10.1177 / 1088868315586325$

Tamir, M., Chiu, C., \& Gross, J. J. (2007). Business or pleasure? utilitarian versus hedonic considerations in emotion regulation. Emotion, 7, 546-554. doi:10.1037/15283542.7.3.546

Tamir, M., \& Ford, B. Q. (2012). When feeling bad is expected to be good: Emotion regulation and outcome expectancies in social conflicts. Emotion, 12, 807-816. doi:10.1037/a0024443

Tamir, M., Ford, B. Q., \& Ryan, E. (2013). Nonconscious goals can shape what people want to feel. Journal of Experimental Social Psychology, 49, 292-297. doi: 10.1016/j.jesp.2012.11.009

Thayer, J. F., Friedman, B. H., \& Borkovec, T. D. (1996). Autonomic characteristics of generalized anxiety disorder and worry. Biological Psychiatry, 39, 255-266. doi: $10.1016 / 0006-3223(95) 00136-0$

Thompson, R. J., Mata, J., Jaeggi, S. M., Buschkuehl, M., Jonides, J., \& Gotlib, I. H. (2012). The everyday emotional experience of adults with major depressive disorder: examining emotional instability, inertia, and reactivity. Journal of Abnormal Psychology, 121, 819829. doi: $10.1037 / \mathrm{a} 0027978$

Trull, T. J., Ebner-Priemer, U. W. (2009). Using experience sampling methods/ecological momentary assessment (ESM/EMA) in clinical assessment and clinical research: 
introduction to the special section. Psychological Assessment, 21, 457-462. doi:

$10.1037 / \mathrm{a} 0017653$

Trull, T. J., Solhan, M. B., Tragesser, S. L., Jahng, S., Wood, P. K., Piasecki, T. M., \& Watson, D. (2008). Affective instability: Measuring a core feature of borderline personality disorder with ecological momentary assessment. Journal of Abnormal Psychology, 117, 647-661. doi: 10.1037/a0012532

Tucker, P., Smith, K. L., Marx, B., Jones, D., Miranda, R. \& Lensgraf, J. (2000). Fluvoxamine reduces physiologic reactivity to trauma scripts in posttraumatic stress disorder. Journal of Clinical Psychopharmacology, 20, 367-372.

Tugade, M. M., \& Fredrickson, B. L. (2004). Resilient individuals use positive emotions to bounce back from negative emotional experiences. Journal of Personality and Social Psychology, 86, 320-333. doi:10.1037/0022-3514.86.2.320

Tull, M. T., \& Aldao, A. (2015). Editorial overview: New directions in the science of emotion regulation. Current Opinion in Psychology, 3, iv-x. doi:10.1016/j.copsyc.2015.03.009

Turk, C. L., Heimberg, R. G., Luterek, J. A., Mennin, D. S., \& Fresco, D. M. (2005). Emotion dysregulation in generalized anxiety disorder: A comparison with social anxiety disorder. Cognitive Therapy and Research, 29, 89-106. doi: 10.1007/s10608-005-1651-1

van de Leemput, I. A., Wichers, M., Cramer, A. O., Borsboom, D., Tuerlinckx, F., Kuppens, P., ... \& Derom, C. (2014). Critical slowing down as early warning for the onset and termination of depression. Proceedings of the National Academy of Sciences, 111, 87-92. doi: 10.1073/pnas.1312114110

van Eck, M., Nicolson, N. A., \& Berkhof, J. (1998). Effects of stressful daily events on mood states: Relationship to global perceived stress. Journal of Personality and Social 
Psychology, 75, 1572-1585. doi: 10.1037/0022-3514.75.6.1572

Vasey, M. W., \& Borkovec, T. D. (1992). A catastrophizing assessment of worrisome thoughts. Cognitive Therapy and Research, 16(5), 505-520. doi:10.1007/BF01175138

Velasco, C. \& Bond. A. (1998). Personal relevance is an important dimension for visceral reactivity in emotional imagery. Cognition and Emotion, 12, 231-242. doi: $10.1080 / 026999398379736$

Vossel, G., \& Zimmer, H. (1990). Psychometric properties of non-specific electrodermal response frequency for a sample of male students. International Journal of Psychophysiology,10(1), 69-73. doi:10.1016/0167-8760(90)90047-H

Watson, D. (1988). The vicissitudes of mood measurement: Effects of varying descriptors, time frames, and response formats on measurement of positive and negative affect. Journal of Personality and Social Psychology, 55, 128-141. doi:10.1037/0022-3514.55.1.128

Watson, D., Clark, L. A., \& Carey, G. (1988). Positive and negative affect and their relation to anxiety and depressive disorders. Journal of Abnormal Psychology, 97, 346-353. doi: 10.1037/0021-843X.97.3.346

Watson, D., Clark, L. A., \& Stasik, S. M. (2011). Emotions and the emotional disorders: A quantitative hierarchical perspective. International Journal of Clinical and Health Psychology, 11, 429-442.

Wells, A. (1999). A metacognitive model and therapy for generalized anxiety disorder. Clinical Psychology and Psychotherapy, 6, 86-95. doi:10.1002/(SICI)10990879(199905)6:2<86::AID-CPP189>3.0.CO;2-S

Westermann, R., Spies, K., Stahl, G., \& Hesse, F. W. (1996). Relative effectiveness and validity of mood induction procedures: A meta-analysis. European Journal of Social 
Psychology, 26, 557-580. doi:10.1002/(SICI)1099-0992(199607)26:4<557::AIDEJSP769>3.0.CO;2-4

Wood, J. V., Heimpel, S. A., Manwell, L. A., \& Whittington, E. J. (2009). This mood is familiar and I don't deserve to feel better anyway: Mechanisms underlying self-esteem differences in motivation to repair sad moods. Journal of Personality and Social Psychology, 96, 363-380. doi: 10.1037/a0012881

Yamasaki, A. S. (2006). Concordance between self-reported and physiological measures of emotion during fear imagery in anxiety disorders (unpublished doctoral dissertation). Pennsylvania State University, Philadelphia, PA.

Yeragani, V. K., Pohl, R., Balon, R., Ramesh, C., Glitz, D., .., Merlos, B. (1992). Effect of imipramine treatment on heart rate variability measures. Neuropsychobiology 26, 27-32. doi: $10.1159 / 000118892$

Zich, J. M., Attkisson, C.C., \& Greenfield, T.K. (1990). Screening for depression in primary care clinics: the CES-D and the BDI. International Journal of Psychiatry in Medicine, 20, 259-277. doi:10.2190/LYKR-7VHP-YJEM-MKM2

Zinbarg, R. E., Craske, M. G., \& Barlow, D. H. (2006). Mastery of your anxiety and worry: Therapist guide (2nd ed.) New York, NY: Oxford University Press. 